NBER WORKING PAPER SERIES

\title{
FIRMS, FAILURES, AND FLUCTUATIONS: THE MACROECONOMICS OF SUPPLY CHAIN DISRUPTIONS
}

\author{
Daron Acemoglu \\ Alireza Tahbaz-Salehi \\ Working Paper 27565 \\ http://www.nber.org/papers/w27565 \\ NATIONAL BUREAU OF ECONOMIC RESEARCH \\ 1050 Massachusetts Avenue \\ Cambridge, MA 02138 \\ July 2020
}

We thank Carlos Molina for excellent research assistance. We are grateful to David Baqaee, Vasco Carvalho, Martin Jensen, Roland Kpodar, Glenn Magerman, Pooya Molavi, Ezra Oberfield, Ali Shourideh, Claudio Tebaldi, Andrea Vedolin, and seminar participants at Boston University, Columbia, FDIC, National Bank of Belgium, Northwestern, Penn, Queen Mary, University of Tokyo, UT-Austin Macro/International Conference, Deutsche Bundesbank-ECB Joint Spring Conference on Systemic Risk and the Macroeconomy, and ERC/LBS/CEPR Conference on Granularity for various comments and suggestions. This research has benefited from financial support from the International Monetary Fund (IMF)-Department for International Development (DFID) project on Macroeconomic Research in Low-Income Countries (Project id: 60925). The views expressed herein are those of the authors and do not necessarily reflect the views of the National Bureau of Economic Research.

NBER working papers are circulated for discussion and comment purposes. They have not been peer-reviewed or been subject to the review by the NBER Board of Directors that accompanies official NBER publications.

(C) 2020 by Daron Acemoglu and Alireza Tahbaz-Salehi. All rights reserved. Short sections of text, not to exceed two paragraphs, may be quoted without explicit permission provided that full credit, including $\odot$ notice, is given to the source. 
Firms, Failures, and Fluctuations: The Macroeconomics of Supply Chain Disruptions

Daron Acemoglu and Alireza Tahbaz-Salehi

NBER Working Paper No. 27565

July 2020

JEL No. D57,E23,E32

\begin{abstract}
$\underline{\text { ABSTRACT }}$
This paper studies how firm failures and the resulting disruptions to supply chains can amplify negative shocks. We develop a non-competitive model where customized supplier-customer relations increase productivity, and the relationship-specific surplus generated between firms and their suppliers is divided via bargaining. Changes in productivity alter the distribution of surplus throughout the economy and determine which firms are at the margin of failure. A firm's failure may spread to its suppliers and customers and to firms in other parts of the production network. We provide existence, uniqueness, and a series of comparative statics results, and show how the response of the equilibrium production network may propagate recessionary shocks.
\end{abstract}

Daron Acemoglu

Department of Economics

MIT

50 Memorial Drive

Cambridge, MA 02142-1347

and NBER

daron@mit.edu

Alireza Tahbaz-Salehi

Kellogg School of Management

Northwestern University

2211 Campus Drive, Evanston, IL 60208

alirezat@kellogg.northwestern.edu 


\section{Introduction}

Businesses are more likely to fail during economic downturns. Figure 1 depicts the annual evolution of firm failures in the United States between 1978 and 2014, with raw and HP-filtered data presented in panels (a) and (b), respectively. The spikes in business failures during the NBER recession dates, shown in gray, are visible in both panels. For example, the failure rate of all U.S. firms increased from an average of $8.3 \%$ in the $2003-2007$ period to more than $9 \%$ during the Great Recession. ${ }^{1}$

One consequence of widespread firm failures is the potential disruption to the efficient operation of supply chains. Supply chains generate productivity gains by enabling input customization, but also add to an economy's fragility: bankruptcies destroy the relationship-specific surplus between firms and their suppliers and may spread in the economy as a firm's failure spills over to its customers, suppliers, and beyond. Such concerns were the motivation for the 2012 U.S. National Strategy for Global Supply Chain Security, which was based on the premise that "[i]ntegrated supply chains are fast and cost-efficient but also susceptible to shocks that can rapidly escalate from localized events into broader disruptions" (The White House, 2012).

The current paper is a first attempt to develop a systematic framework for studying how firm failures and the resulting supply chain disruptions impact the macroeconomy and amplify recessionary shocks. Modeling the relationship-specific surplus that is at the heart of supply chains requires a departure from competitive analysis. We achieve this by developing a tractable framework in which firms and their suppliers bargain over their relationship-specific surplus. In this framework, changes in productivity alter the distribution of this surplus throughout the economy, determine which firms are at the margin of failure, and may trigger cascading failures impacting the entire production network of the economy. ${ }^{2}$

We consider a static economy consisting of $n$ intermediate sectors that produce with labor and inputs from other sectors and whose products are then combined into a unique consumption good by a final good sector. Each industry comprises two types of producers. The first is a competitive fringe of firms that produce a "generic" variant of the good using a constant returns technology with inputs sourced from other generic producers. This part of the economy thus defines a sectoral production network and is similar to a standard input-output economy (e.g., Long and Plosser, 1983; Acemoglu et al., 2012). The second group of firms, which we refer to as "customized" producers, have access to a more productive technology, provided that they pay a fixed cost of operation and use inputs from their designated customized suppliers. ${ }^{3}$ The relationship-specific productivity gains generated by customized firms and their potential loss due to shocks or firm failures are our points of departure from the previous literature.

While generic producers are price-takers and generic prices are determined competitively, the

\footnotetext{
${ }^{1}$ Appendix E provides information on data sources and additional results confirming the patterns shown here, including a very similar cyclical behavior for exit rate of establishments (as opposed to firms) in the United States. Also see Chen (2010), who documents similar patterns for movements in default rates and credit spreads over the business cycle.

${ }^{2}$ Unless otherwise noted, we use the term "production network" to refer to the network of firm-level input-output linkages and "supply chain" to refer to a firm's collection of direct and indirect suppliers and customers. We also use "failure" and "exit" interchangeably.

${ }^{3}$ We simplify our model by taking the matches between firms and their suppliers as given and abstracting from the formation of firm-specific relationships. See the discussion in Subsection 5.2.
} 


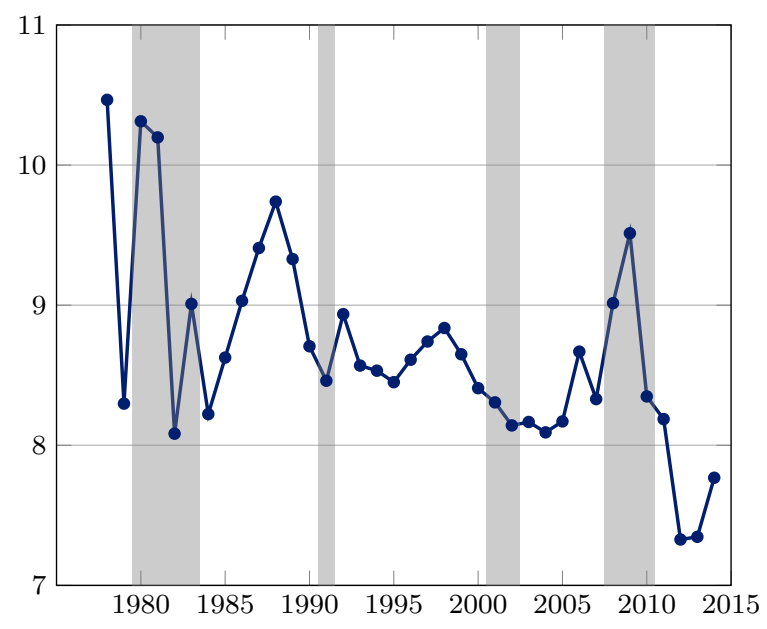

(a) raw data

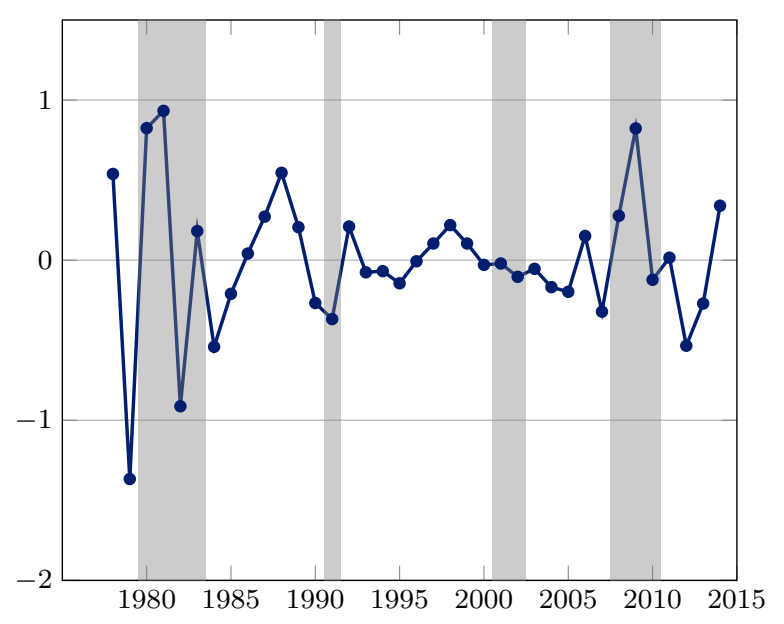

(b) HP-filtered data

Figure 1. Annual Firm Exit Rate in the United States over the Business Cycle

Data source: Business Dynamics Statistic, United States Census Bureau.

Notes: The figure depicts the annual exit rate of firms in the U.S. between 1978 and 2014. Exit rate in year $t$ is defined as the number of firm failures in year $t$ divided by the average number of surviving firms in years $t-1$ and $t$. Panel (a) plots the raw data, whereas panel (b) plots the detrended series using a Hodrick-Prescott filter with smoothing parameter 6.25. Year $t$ is shaded if there is an NBER recession at some point between the second quarter of year $t-1$ and the first quarter of year $t$.

same is not true for customized producers. Instead, we assume that the relationship-specific surplus generated by customization is shared between suppliers and customers via a bargaining protocol à la Rubinstein (1982). These bargains determine customized firms' equilibrium prices and markups. A full equilibrium consists of profit-maximizing input and output decisions by all firms, market-clearing generic prices, equilibrium bargained prices for customized products, and profit-maximizing exit decisions for customized producers.

Negative shocks to this economy-in the form of lower productivity or higher fixed costs of operation for some firms, sectors, or in the aggregate-alter the distribution of surplus throughout the production network and can push customized firms into failure (because they cannot cover their fixed costs). ${ }^{4}$ A customized firm can fail as a direct consequence of a negative shock to its production technology. Or it can fail indirectly because either (i) some of its suppliers go out of business, forcing it to switch to less productive generic suppliers; (ii) its customer suffers a negative shock, fails, or simply reduces its demand, depriving the firm of the revenue necessary for covering its fixed cost; or (iii) losses in other parts of the network forces the firm to reduce its markup and profits. Any additional failure can reduce surviving firms' profits and lead to a new round of failures, generating a potentially powerful amplification mechanism. Figure 2 provides a stylized example of these propagation mechanisms.

We analyze this economy first under the assumption that production technologies are Leontief and then under more general production functions exhibiting complementarities between inputs. After establishing equilibrium existence, we turn to comparative static results on how negative shocks

\footnotetext{
${ }^{4}$ Although we use the language of negative shocks, our analysis is in terms of (full information) comparative statics. We clarify how these changes can be viewed as shocks from the viewpoint of earlier decisions in Subsection 5.2.
} 


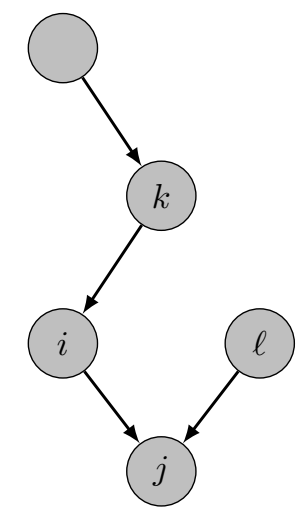

Figure 2. Propagation of Shocks in a Stylized Economy

Notes: This figure illustrates the potential consequences of negative shocks in a stylized economy. Firm $i$ may fail as a result of negative productivity shocks to any of the other firms in the economy. A negative shock to its customer firm $j$ may result in $i$ 's failure by reducing its revenue, while a negative shock to its supplier firm $k$ may result in $i$ 's failure by increasing its marginal cost. Firm $i$ may also fail following a negative shock to firm $\ell$ that reduces the total surplus in the economy and hence $i$ 's profits. Analogously, a negative shock to firm $i$ may lead to the failures of firms $j, k$, and $\ell$.

impact firm-level prices, quantities, markups, firm failures, and aggregate variables such as GDP. Before describing these results, it is useful to clarify the two main channels via which shocks affect economic activity in our framework. More specifically, we note that the aggregate impact of, say, a productivity shock that changes the vector of productivities from $\bar{A}$ to $\underline{A}$ can be decomposed as follows:

$$
\Delta \mathrm{GDP}=\left.\Delta \mathrm{GDP}\right|_{\overline{\mathbf{G}}^{*}, \bar{A} \rightarrow \underline{A}}+\left.\Delta \mathrm{GDP}\right|_{\overline{\mathbf{G}}^{*} \rightarrow \underline{\mathbf{G}}^{*}, \underline{A}},
$$

where $\overline{\mathbf{G}}^{*}$ and $\underline{\mathbf{G}}^{*}$ denote, respectively, the equilibrium (firm-level) production networks before and after the shock. The first term on the right-hand side of (1) represents the response of GDP to a change in productivity holding the network fixed. This response includes any effects arising from the propagation of the shock over input-output linkages working through changes in prices and quantities (e.g., as in Long and Plosser (1983) and Acemoglu et al. (2012) in a competitive framework without markups or in Jones (2013) and Baqaee and Farhi (2020b) in the presence of markups). ${ }^{5}$ Our main focus, however, is on the second term on the right-hand side of (1). This term represents the impact of changes in the economy's production network and hence incorporates any effects working through firm failures.

Our comparative static results come in two flavors. In our first set of results, we focus on the first term on the right-hand side of equation (1) and characterize how shocks impact GDP via changes in markups and prices when the firm-level production network is assumed to be exogenous. While not the main focus of our analysis, these results are, to the best of our knowledge, novel, as they characterize the impact of various shocks when markups throughout the production network adjust.

\footnotetext{
${ }^{5}$ This term already includes some of the new economic forces introduced in our model. While Baqaee and Farhi (2020b) provide a decomposition of aggregate impact of shocks with changing markups, such a decomposition, by its nature, does not endogenize the response of markups to productivity (or other) shocks and can only be computed with sufficiently detailed data on markups both before and after the relevant shocks are realized. In contrast, our model incorporates markups' endogenous response to shocks.
} 
We establish that an increase in the productivity of a subset of customized firms increases GDP, but at the same time raises markups. The increase in markups in response to positive productivity shocks is a consequence of the non-competitive nature of the economy: pairwise bargaining between suppliers and customers transforms some of the gains from higher productivity into higher markups throughout the economy. The effect of productivity shocks on firm profits is more complex. We show that while the firm experiencing the productivity increase and all its downstream customers enjoy greater profits, the profits of its upstream suppliers may decrease. This is because of a "perverse quantity effect"-the greater productivity of the focal firm implies that it demands less inputs from its suppliers, which may reduce supplier profits. This non-monotone impact of productivity shocks on profits undergirds richer responses once we endogenize the equilibrium production network.

In our second set of comparative static results, we turn to the endogenous response of the production network to shocks. We first show that an increase in fixed costs always results in more failures, reduces GDP, and pushes down the profits and markups of all surviving firms. The equilibrium response of the production network, corresponding to the second term in equation (1), amplifies the shock's impact as initial failures translate into more widespread supply chain disruptions. In particular, firm $i$ 's failure reduces the profits of its (direct and indirect) suppliers and customers as well as possibly other firms in the economy.

The implications of higher productivity in the presence of endogenous production networks are more nuanced. While an increase in the productivity of firms with no active suppliers increases profits and GDP and reduces failures, the same may not be true when firms experiencing the productivity increase have customized suppliers. Because of the above-described perverse quantity effect, productivity improvements may reduce demand for and profits of upstream firms and thus trigger a series of failures, which may then undo the positive direct effects of the productivity increase. In such a scenario, positive shocks may be recessionary.

Taken together, our paper provides a tractable model of the distribution of surplus (or "quasirents") in supply chains and shows how this distribution plays a central role in propagating shocks over the production network, determines the extent and nature of firm failures, and mediates the macroeconomic impact of shocks. ${ }^{6}$ Our analysis clarifies how supply chains generate a novel type of fragility and why their equilibrium response amplifies economic downturns.

Related Literature: Our paper is most closely related to the growing literature that studies the role of input-output networks as a mechanism for propagation and amplification of shocks. ${ }^{7}$ It is useful to separate this literature into three branches. The first branch investigates propagation through sectoral input-output networks. The main economic effects in this body of work are mediated by changes in

\footnotetext{
${ }^{6}$ The reference to "quasi-rents" is to emphasize that what matters for propagation in our model is the changes in gross profits out of which firms have to cover their fixed costs of operation.

${ }^{7}$ This literature may in turn be placed in the larger body of works studying the microeconomic origins of macroeconomic fluctuations, such as Gabaix (2011) and Carvalho and Gabaix (2013), who emphasize the role of firm size distribution in translating micro shocks into macro fluctuations. There is also a growing empirical literature that documents the propagation of shocks over production networks, including Acemoglu, Akcigit, and Kerr (2016) at the industry level and Barrot and Sauvagnat (2016), Carvalho, Nirei, Saito, and Tahbaz-Salehi (2017), and Boehm, Flaaen, and Pandalai-Nayar (2019) at the firm level. See Carvalho and Tahbaz-Salehi (2019) for a recent survey of the theoretical and empirical literature on production networks.
} 
prices and quantities. For example, in the context of the competitive equilibrium studied in Long and Plosser (1983) and Acemoglu et al. (2012), productivity shocks propagate due to changes in firms' marginal costs and relative input prices. ${ }^{8}$ We depart from this literature in two significant ways. First, our focus is explicitly on disruptions to supply chains and how destruction of valuable productive relationships due to failures may have non-trivial aggregate implications. Second, our departure from the "competitive framework" - and its variants with exogenously-specified wedges/markupsimplies that the nature of propagations in our model is different from the above-mentioned literature: instead of changes in prices and quantities, shocks in our non-competitive (bargaining) framework are propagated via changes in the distribution of firms' surplus (or quasi-rents) that force some firms into the failure region. An important consequence of these differences is that our model generates rich propagation patterns resulting in upstream, downstream, and horizontal propagation of failures.

A second branch of this literature studies the endogenous formation of production networks, either at the industry level (e.g., Carvalho and Voigtländer (2015) and Acemoglu and Azar (2020)) or at the firm level (e.g., Oberfield (2018), Lim (2018), and Huneeus (2019)). These papers only focus on adjustments on the intensive margin and abstract from firm failures and the macroeconomic effects of supply chain disruptions-mechanisms that are the main focus of our study.

Our paper is most closely related to a third (and smaller) branch of this literature that studies how extensive margin adjustments in production networks propagate shocks in the macroeconomy. For example, Baqaee (2018) and Baqaee and Farhi (2020a) endogenize the number of firms in each industry via a free-entry condition in a model of industry-level production networks and exogenous markups. Taschereau-Dumouchel (2020) studies a model of firm failures in which operating decisions of nearby firms are strategic complements. Finally, the recent independent work by Elliott, Golub, and Leduc (2020) studies macroeconomic fragility in a reduced-form model of failure cascades. We depart from these studies in two significant ways. First, we develop a framework with endogenous markups and division of relationship-specific surplus. Second, we focus on how changes in the distribution of quasi-rents and dissolution of firm-specific relationships in supply chains shape macroeconomic outcomes. $^{9}$

Last but not least, our paper is also related to the vast literature on firm entry and exit. On the empirical side, papers such as Dunne, Roberts, and Samuelson (1988), Foster, Haltiwanger, and Syverson (2008), and Tian (2018) study firm entry and exit over the business cycle. Most of the theoretical papers in this area (such as Campbell (1998), Bilbiie, Ghironi, and Melitz (2012), Clementi and Palazzo (2016), and Carvalho and Grassi (2019)) build on the seminal papers by Jovanovic (1982) and Hopenhayn (1992). For the most part, these works do not consider the role of firm failures in

\footnotetext{
${ }^{8}$ The same mechanism is at work in papers such as Jones (2013), Liu (2019), Baqaee and Farhi (2020b), and Bigio and La'O (2020), who study the propagation of shocks in the presence of exogenously-specified markups/wedges that do not adjust in response to shocks. An exception is the recent work by Kikkawa, Magerman, and Dhyne (2020), where firms charge customer-specific markups that depend on bilateral input shares.

${ }^{9}$ Also see Carvalho, Elliott, and Spray (2020), who study "bottleneck firms," whose removal from the supply chain results in a substantial decline in aggregate productivity. A related body of works studies how the propagation of shocks over financial networks and the resulting failures of financial institutions can generate systemic risk (e.g., Allen and Gale (2000), Elliott, Golub, and Jackson (2014), and Acemoglu, Ozdaglar, and Tahbaz-Salehi (2015)). While this literature shares our focus on the aggregate implications of firm failures, the nature of bankruptcies in these models is different: they are driven by counterparty risk and are tightly linked to the specifics of inter-bank lending contracts. The implications of such failures for GDP and macroeconomic efficiency have not been the focus of this literature either.
} 
destroying valuable inter-firm relationships and disrupting supply chains. One feature highlighted by the empirical and theoretical works in this area is that exiting firms are generally less productive (as they are being pushed out in response to small shocks). This is not necessarily the case in our model (nor is it always the case in recessions, as documented by Foster, Grim, and Haltiwanger (2016)), as highly productive firms can be forced out due to shifts in the distribution of surplus across the economy or, in the extreme, because of a collapse in their supply chains. This difference is part of the reason why firm failures in our framework can serve as a major driver of economic downturns.

Outline: The rest of the paper is organized as follows. We present the model in Section 2 and define our solution concept in Section 3. Section 4 characterizes the equilibrium and presents our main comparative static results under the assumption that all firms use Leontief production technologies. We extend these results to a general class of production functions with complementarities across inputs in Section 5. Section 6 concludes the paper. Appendix A presents a new fixed point theorem, which we use for establishing the existence of equilibrium, and Appendix B contains the proofs of the main results in the text. The remaining proofs, further results, and data sources and details for Figure 1 are provided in a series of online appendices.

\section{Model}

Consider an economy consisting of $n+1$ industries and lasting for three periods $t=0,1,2$. Industries labeled $\{1,2, \ldots, n\}$ produce intermediate goods, while the industry labeled 0 produces a final product that is sold to households.

Each intermediate industry $I$ comprises two types of firms: (i) a competitive fringe that produces a generic variant of industry I's good and (ii) a finite collection of firms, each of which produces a variant that is customized to the production technology of a specific firm. Thus, $I=\left\{i^{b}\right\} \cup\left\{i_{1}^{a}, \ldots, i_{k_{I}}^{a}\right\}$, where $i^{b}$ stands for the representative firm that produces the generic variant ("generic producer" for short) and $\left\{i_{1}^{a}, \ldots, i_{k_{I}}^{a}\right\}$ denote producers of customized variants ("customized producers"). ${ }^{10}$ While customized producers have to pay a fixed cost at $t=0$ to be able to operate, generic producers face no such costs. All firms in an industry use the same constant returns production technology but may have different productivities.

The industry labeled 0 consists of a collection of identical firms that face no fixed costs and can transform the intermediate inputs from the generic and customized producers into a single final product, which is then sold to the households. Throughout, we take this final good as the numeraire. The representative household has utility function $u(\cdot)$ over the final good and inelastically supplies $L$ units of labor, which is the only factor of production.

The timing of the model is as follows. At $t=0$, each customized producer decides whether to pay a fixed cost of operation. At $t=1$, customized producers that pay this fixed cost, alongside generic producers and the final good producers in industry 0 , enter into pairwise contracts that determine

\footnotetext{
${ }^{10}$ Throughout, we use uppercase letters to denote industries and lowercase letters to denote individual firms, with the convention that firms indexed $i, i^{a}$, and $i^{b}$ belong to industries indexed with the corresponding uppercase letter, $I$.
} 
prices. Production and consumption take place at $t=2$. In what follows, we describe each of the model ingredients in further detail.

\subsection{Generic Producers}

A representative firm $i^{b}$ in each industry $I$ produces the generic variant of industry $I$ 's output using a constant returns technology with labor and other generic goods as inputs. This technology, which we refer to as the $B$-technology, is described by the cost function

$$
c_{i}^{b}=C_{I}\left(w, \frac{p_{1}^{b}}{B_{i 1}}, \ldots, \frac{p_{n}^{b}}{B_{i n}}\right)
$$

where $p_{j}^{b}$ is the price of the generic variety of the product of industry $J$ (produced by representative firm $j^{b}$ ), $B_{i j}$ denotes the input-augmenting productivity level, $w$ denotes the wage, and the cost function $C_{I}$ is homogenous of degree 1 .

\subsection{Customized Producers}

Industry $I$ additionally includes a finite collection of customized producers, $\left\{i_{1}^{a}, \ldots, i_{k_{I}}^{a}\right\}$, each of which can produce a distinct variant of the good by combining labor and the output of other customized producers and/or generic producers. In what follows, we drop the superscript $a$ for customized producers whenever there is no risk of confusion.

Unlike the output of generic producers, which can be used as an input by any firm in the economy, the variants produced by customized producers can only be used by specific firms as intermediate inputs for production. We capture these firm-specific relationships by means of a firm-level network of technological compatibilities, $\mathcal{G}$, whose vertices correspond to the set of all customized intermediate and final good producers in the economy, while a directed edge $(j, i)$ is present from vertex $j$ to vertex $i$ if the output of customized producer $j$ can be used as an intermediate input by firm $i .{ }^{11} \mathcal{G}$ may represent pairwise relationship-specific investments made in earlier stages, reputation, or trust between firms that enable customized relationships (see Subsection 5.2).

Assumption 1. The network of technological compatibilities, $\mathcal{G}$, satisfies the following conditions:

(i) each firm in $\mathcal{G}$ has at most one customer;

(ii) each firm in $\mathcal{G}$ has at most one customized supplier in any given industry.

This assumption restricts each customized firm in $\mathcal{G}$ to have at most one customer, but a firm may have multiple customized suppliers as long as they belong to different industries. We discuss the role of Assumption 1 in our analysis below, but here note that it allows for significant levels of firmlevel heterogeneity in $\mathcal{G}$ : customized producers in one industry may be designated suppliers of firms in different industries or belong to supply chains with very different structures. As we show in the following sections, this heterogeneity translates into heterogenous prices, markups, and differential

\footnotetext{
${ }^{11}$ We do not include the generic producers as part of $\mathcal{G}$. However, as we describe shortly, generic variants can be used as an input by all customized, generic, and final good producers in the economy.
} 
responses to shocks. Finally, while $(j, i) \in \mathcal{G}$ means that the variant produced by $j$ can be used as an input by $i$, such a trade need not take place in equilibrium, as the customer firm $i$ may opt for using the generic variant $j^{b}$ instead, or either firm may exit.

Customized producers operate constant returns production technologies that transform labor and generic and customized variants to output. In particular, the technology employed by a customized producer $i \in I$, which we refer to as the A-technology, is described by the cost function

$$
c_{i}^{a}=C_{I}\left(w,\left\{\min \left\{\frac{p_{i j}^{a}}{A_{i j}}, \frac{p_{j}^{b}}{B_{i j}}\right\}\right\}_{(j, i) \in \mathcal{G}},\left\{\frac{p_{j}^{b}}{B_{i j}}\right\}_{(j, i) \notin \mathcal{G}}\right),
$$

where $C_{I}$ coincides with the production function of generic producers in (2), $w$ is the market wage, $p_{i j}^{a}$ is the input price of the variant produced by a customized producer $j, p_{j}^{b}$ is the price of the generic variant produced by (generic) producer $j^{b}$, and $A_{i j}$ and $B_{i j}$ are input-augmenting productivities of the customized and generic inputs, respectively. According to (3), a customized producer $i$ always has the ability to use generic variants as intermediate inputs. In addition, if there exists a customized producer $j \in J$ such that $(j, i) \in \mathcal{G}$, then $i$ can also produce using $j$ 's customized variant instead of the generic input from $j^{b}$. We assume:

Assumption 2. $A_{i j} \geq B_{i j}$ for all pairs $(j, i) \in \mathcal{G}$.

The wedge between $A_{i j}$ and $B_{i j}$ captures the extent of input-specificity between customized firm $i$ and its customized supplier $j$.

In order to operate its technology, each customized firm $i \in I$ has to pay a fixed cost $z_{i} \geq 0$ in units of labor at $t=0$, which is sunk before the start of the contracting stage at $t=1$. Customized firms that pay this cost can produce using the technology in (3) at $t=2$, whereas firms that do not pay this fixed cost exit or "fail". We use $\mathbf{G} \subseteq \mathcal{G}$ to denote the subnetwork induced by the set of active firms. Thus, unlike the exogenously-specified network of technological compatibilities, $\mathcal{G}$, the firm-level network $\mathbf{G}$, which we refer to as the economy's production network, is determined in equilibrium. Finally, we say that production network $\mathbf{G}$ is feasible if the aggregate fixed cost—measured in units of labor-for the set of active firms in $\mathbf{G}$ does not exceed the aggregate supply of labor $L$.

\subsection{Final Good Producers}

Firms in industry 0 transform inputs from industries $\{1, \ldots, n\}$ into a final product using a constant returns production technology. Firms in this industry are of two types as well: a competitive fringe of generic producers and a collection of customized producers, with respective production technologies:

$$
\begin{aligned}
c_{0}^{b} & =C_{0}\left(p_{1}^{b}, \ldots, p_{n}^{b}\right) \\
c_{0}^{a} & =C_{0}\left(\left\{\min \left\{p_{0 i}^{a}, p_{i}^{b}\right\}\right\}_{(i, 0) \in \mathcal{G}},\left\{p_{i}^{b}\right\}_{(i, 0) \notin \mathcal{G}}\right) .
\end{aligned}
$$

As with other industries, the generic final good producer can only produce using generic inputs, whereas the customized producers can also use the variant produced by their designated customized suppliers. We assume that customized producers in industry 0 face no fixed cost of operation and have no bargaining power vis-à-vis any of their customized suppliers. 


\subsection{Contracts and Terms of Trade}

At $t=1$, all active firms - namely, generic producers, final good producers, and customized producers that have paid the fixed costs-can enter into pairwise contracts specifying the prices at which trades take place at $t=2$.

Since generic producers are competitive, they price at marginal cost regardless of whether the (representative) generic producer $i^{b}$ supplies to customized, generic, or final good producers. Therefore, $p_{i}^{b}=c_{i}^{b}$, where the marginal cost $c_{i}^{b}$ is given by (2).

In contrast, the unit price at which a potential supplier-customer pair $(j, i) \in \mathbf{G}$ of customized producers trade with one another is determined at $t=1$ via pairwise bargaining à la Rubinstein (1982). Specifically, we assume that period $t=1$ consists of infinitely many sub-periods and that at any given sub-period, the supplier firm $j$ is selected with an exogenously-specified probability $\delta_{i j}$ to make a price offer $p_{i j}^{a}$ to its customer $i$, while the customer makes an offer to the supplier with the complementary probability of $1-\delta_{i j}$. If one party accepts the other's offer, the agreement $p_{i j}^{a}$ is implemented and the supplier commits to delivering as many units of its product as demanded by the customer at $t=2$ at the fixed unit price of $p_{i j}^{a}$. If an offer is rejected, a new proposer is selected at the next sub-period to make a new offer according to the same protocol. The two parties discount future sub-periods at a common rate $\eta<1$, and we take $\eta \rightarrow 1$. After rejecting an offer, each party also has the outside option of walking away with no agreement, in which case the two parties cannot trade at $t=2$.

A few remarks are in order. First, the recognition probability $\delta_{i j}$ captures the relative bargaining power of the customized supplier vis-à-vis its customer, and thus $\delta_{i j}=1$ corresponds to the extreme case in which firm $j$ has all the bargaining power and makes take-it-or-leave-it offers to customer $i$. This observation illustrates that the collection of $\left\{\delta_{i j}\right\}$ 's plays a central role in determining firm-level markups, the markups' response to shocks, and hence the shocks' pass-through. Second, due to the nature of the production technology in (3), customer $i$ always has access to an outside option of using the generic variant $j^{b}$ as an input for production. As a result, any price offer $p_{i j}^{a}$ that exceeds $p_{i j}^{b} A_{i j} / B_{i j}$ is rejected by customer $i{ }^{12}$ In addition, the fact that both parties have the outside option of walking away means that (i) they reach an agreement in equilibrium only if there are gains from trade; (ii) the agreement price $p_{i j}^{a}$ cannot fall below $j$ 's marginal cost; and (iii) $i$ 's marginal cost at the agreement price $p_{i j}^{a}$ does not exceed its output price (so that it does not make negative profits).

\section{Equilibrium}

In this section, we define our solution concept. Recall that customized firms pay the fixed costs of operation at $t=0$, pairwise prices are determined via a bargaining process at $t=1$, and production and consumption decisions take place at $t=2$. We therefore proceed by defining and characterizing the equilibrium recursively using backward induction.

We first focus on the economy at $t=2$, when the set of active firms, the production network $\mathbf{G}$, and all (generic and customized) prices are already determined. We use $\boldsymbol{p}^{b}=\left(p_{1}^{b}, \ldots, p_{n}^{b}, w\right)$ and $\boldsymbol{p}^{a}=$

\footnotetext{
${ }^{12}$ We assume that for firm $i$ to take this outside option, it must first reject $j$ 's offer. This timing assumption is necessary to guarantee uniqueness of the bargaining outcome. See Shaked (1987) and Osborne and Rubinstein (1990, p.55) for more details.
} 
$\left(p_{i j}^{a}\right)_{(j, i) \in \mathbf{G}}$ to denote the vectors of generic and customized prices in the economy, with the convention that $p_{i j}^{a}=\varnothing$ if the pair $(j, i) \in \mathbf{G}$ has not reached an agreement.

Definition 1. Given a feasible production network of active firms $\mathbf{G}$ and the vector of prices $\left(\boldsymbol{p}^{a}, \boldsymbol{p}^{b}\right)$, a production equilibrium is a collection of input and output quantities and consumption such that

(i) all firms minimize production costs while meeting their output obligations to their customers;

(ii) the representative household maximizes her utility;

(iii) all markets clear.

The notion of production equilibrium takes the set of active firms and all prices as given: at $t=2$, all (generic and customized) producers are committed to delivering as many units of output as demanded by their customers at prices agreed upon at $t=1$. Thus, the only requirement the production equilibrium imposes is that firms minimize costs while meeting their output obligations. Market clearing then guarantees that firms' and the representative household's quantity decisions are mutually consistent. We now proceed to the contracting stage at $t=1$ when prices are determined.

Definition 2. Given a feasible production network $\mathrm{G}$, a bargaining equilibrium is a collection of prices $\left(\boldsymbol{p}^{a}, \boldsymbol{p}^{b}\right)$ and quantities such that

(i) the quantities in any ensuing subgame correspond to a production equilibrium;

(ii) given $\left(\boldsymbol{p}^{a}, \boldsymbol{p}^{b}\right)$, no generic producer $i^{b}$ can earn higher profits by offering a different price;

(iii) for each supplier-customer pair of firms $(j, i) \in \mathbf{G}$, the bilaterally-agreed price $p_{i j}^{a}$ is (part of) a Subgame Perfect Nash Equilibrium of the pairwise bargaining game between $i$ and $j$, taking all other prices and household's income as given.

The first condition in Definition 2 imposes that all firms minimize costs at $t=2$ and all markets clear. The other two conditions ensure that neither the generic nor the customized producers can earn higher profits in the subsequent subgames by deviating from the prices prescribed by the equilibrium. Condition (ii) is equivalent to generic prices being determined in the competitive equilibrium (but we state it in the form of a "no deviation" requirement in analogy with the rest of the definition). Condition (iii), on the other hand, requires, that each pair of customized firms plays subgame perfect strategies in the bargaining game (and with a slight abuse of terminology, we refer to the agreed price as "part of" SPNE). More specifically, this condition imposes that any unilateral deviation, either by making a different offer, taking the outside option, or walking away, should not be profitable. ${ }^{13}$

Our final definition of this section endogenizes the production network at $t=0 .{ }^{14}$

\footnotetext{
${ }^{13}$ By taking the income level of the representative household as given, each firm ignores the potential impact of its bargained price on aggregate demand working through changes in profits and household wealth. Since there is a finite number of customized firms, this effect is in general nonzero, but to the extent that the number of firms is large, it would be small. All our results continue to apply without any modification when firms recognize this effect, but the analysis in the case of general production technologies in Subsection 5.1 becomes more cumbersome. Whether this effect is recognized or not is immaterial for the definition of a full equilibrium, presented in Definition 3.

${ }^{14}$ With some abuse of notation, we use $\mathbf{G}^{*}$ to denote both the production network and its vertex set.
} 
Definition 3. A full equilibrium consists of a production network $\mathbf{G}^{*} \subseteq \mathcal{G}$ and collections of prices and quantities such that

(i) the quantities correspond to a production equilibrium in the corresponding subgames at $t=2$;

(ii) the prices correspond to a bargaining equilibrium in the corresponding subgames at $t=1$;

(iii) no customized firm has an incentive to change its exit decision at $t=0$, i.e.,

$$
\begin{array}{ll}
\pi_{i}\left(\mathbf{G}^{*}\right)-z_{i} w \geq 0 & \forall i \in \mathbf{G}^{*} \\
\pi_{i}\left(\mathbf{G}^{*} \cup\{i\}\right)-z_{i} w<0 & \forall i \notin \mathbf{G}^{*},
\end{array}
$$

where $\pi_{i}(\mathbf{G})$ is the profit of firm $i$ (gross of fixed costs) when the network of active firms is $\mathbf{G}$.

The full equilibrium endogenizes the (firm-level) production network by requiring that exit decisions at time $t=0$ be profit maximizing while anticipating the equilibrium play at $t=1$ and $t=2$. In particular, each firm recognizes how its decision to remain active impacts prices and quantities in the subsequent bargaining and production stages.

\section{Equilibrium Analysis}

In this section, we provide a detailed equilibrium characterization and provide comparative statics for the micro and macroeconomic impacts of various shocks. In addition, we present a decomposition of the response of GDP into a component due to changes in prices and quantities along a given production network and a component capturing the endogenous adjustment of the production network.

Throughout this section, we impose:

Assumption 3. All production technologies are Leontief, i.e., $C_{I}\left(w, p_{1}, \ldots, p_{n}\right)=w+\sum_{j=1}^{n} p_{j}$.

This assumption, which implies that firms use inputs in fixed proportions, enables us to characterize the equilibrium in closed-form and present the model's underlying economic mechanisms in the most transparent manner. We extend our results to a general class of production functions in Section 5.

\subsection{Production and Bargaining Equilibria}

As our first result, we establish the existence and uniqueness of the production and bargaining equilibria and characterize these equilibria in terms of model primitives and the production network G. As already anticipated, a key object in our analysis is the collection of markups charged by customized firms. It turns out to be more convenient to work with the customized firms' "absolute markups", defined as the difference between their prices and marginal costs. We use $\mu_{i}$ to denote firm $i$ 's absolute markup, which for simplicity (and with a slight abuse of terminology), we refer to as its markup.

Theorem 1. Suppose Assumptions 1-3 are satisfied. Then, 
(a) for any feasible production network $\mathbf{G}$ and collection of prices $\left(\boldsymbol{p}^{a}, \boldsymbol{p}^{b}\right)$, the production equilibrium exists and is unique;

(b) for any feasible production network $\mathrm{G}$, the bargaining equilibrium exists and is unique (up to a scaling) and all pairs of firms $(j, i) \in \mathbf{G}$ reach an agreement in equilibrium;

(c) for any feasible production network $\mathbf{G}$, the equilibrium vector of generic prices $\boldsymbol{p}^{b}=\left(p_{1}^{b}, \ldots, p_{n}^{b}, w\right)$ is the unique solution to the system of equations

$$
p_{i}^{b}=w+\sum_{j=1}^{n} p_{j}^{b} / B_{i j}
$$

and the vector of customized prices $\boldsymbol{p}^{a}=\left(p_{i j}^{a}\right)_{(j, i) \in \mathbf{G}}$ is the unique solution to the system of equations

$$
\min \left\{\delta_{i j} A_{i j} \mu_{i}-\left(1-\delta_{i j}\right) \mu_{j}, p_{j}^{b}\left(A_{i j} / B_{i j}\right)-p_{i j}^{a}\right\}=0
$$

for all supplier-customer pairs $(j, i) \in \mathbf{G}$.

Statements (a) and (b) of Theorem 1 establish the existence and uniqueness of production and bargaining equilibria for arbitrary production networks (and for any distribution of bargaining powers and productivity levels). Statement (b) additionally shows that any pair of active firms with the opportunity to trade reach an agreement in equilibrium. This is a natural consequence of Assumption 2 , which ensures that customized technologies are superior to generic technologies. ${ }^{15}$

The last part of Theorem 1 provides a system of equations that characterizes generic and customized prices in terms of the production network of active firms $\mathbf{G}$, productivity shocks, and the customized firms' bargaining powers. Equation (4) reiterates that generic prices $\boldsymbol{p}^{b}$ are determined by marginal costs and are independent of the set of active customized firms in $\mathrm{G}$, their bargaining power, and the customized productivities $A_{i j}$. In contrast, customized prices, given as a solution to equation (5), depend on the details of the bargaining process as well as generic and customized productivities.

Equation (5) has a simple interpretation. First, it guarantees that the agreement price $p_{i j}^{a}$ between a pair of active customized firms $(j, i) \in \mathbf{G}$ can never exceed $p_{j}^{b} A_{i j} / B_{i j}$, since otherwise the customer would prefer to exercise her outside option and use the generic variant of this input. Second, it implies that when this outside option does not bind (i.e., when $p_{i j}^{a}<p_{j}^{b} A_{i j} / B_{i j}$ ), the firms split the gross surplus from their relationship-specific productivity, $A_{i j}$, in proportion to their bargaining powers:

$$
\frac{\mu_{j}}{\mu_{i}}=\left(\frac{\delta_{i j}}{1-\delta_{i j}}\right) A_{i j}
$$

where recall that $\delta_{i j}$ captures the bargaining power of the supplier $j$. Therefore, all else equal, a higher $\delta_{i j}$ increases $j$ 's share of the pairwise productivity gross surplus_by raising the price of $j$ 's price $p_{i j}^{a}$ for firm $i .{ }^{16}$ Finally, whether the outside option in (5) binds depends on not just the pairwise productivity $A_{i j}$, but also productivities in other parts of the economy.

\footnotetext{
${ }^{15}$ There may be instances of no agreement off the equilibrium path. This is because, under non-equilibrium price vectors, productivity gains from customized production may be insufficient to make agreement worthwhile for both parties.

${ }^{16}$ The reason the ratio of markups in (6) only depends on the bargaining powers and the pairwise productivity $A_{i j}$ (but not the gap between the customized and generic productivities) is because of our bargaining protocol, which ensures that, in contrast to the Nash solution to bargaining, outside options only matter when they are binding. See, for example, Osborne and Rubinstein (1990, Section 3.12).
} 
Overall, equations (4) and (5) provide a relatively simple system of equations pinning down prices and markups in terms of the production network and all productivities in the economy. Importantly, they also extend Samuelson's (1951) "non-substitution theorem” to our non-competitive environment with firm-level bargaining: equilibrium prices $\left(\boldsymbol{p}^{a}, \boldsymbol{p}^{b}\right)$ are independent of household preferences, the production technology for the final good, and equilibrium quantities. ${ }^{17}$ This non-substitution theorem, like its classical variant for competitive economies, enables us to investigate the impact of shocks on equilibrium prices and markups without solving for quantities.

\subsection{Full Equilibrium}

We now turn to the economy's full equilibrium, which endogenizes the production network. Recall from Definition 3 that, in a full equilibrium, a customized firm $i$ chooses to remain active if and only if its profits exceed its fixed cost of operation. This requirement can be restated as a fixed point of the mapping:

$$
\phi(\mathbf{G})=\left\{i \in \mathcal{G}: \pi_{i}(\mathbf{G} \cup\{i\}) \geq w z_{i}\right\},
$$

which maps a collection of active firms $\mathbf{G} \subseteq \mathcal{G}$ to the set of firms that would make non-negative net profits if they paid the fixed cost. Thus, $\mathbf{G}^{*} \subseteq \mathcal{G}$ is part of a full equilibrium if and only if $\mathbf{G}^{*}=\phi\left(\mathbf{G}^{*}\right)$. We can therefore characterize the economy's full equilibria by determining the set of fixed points of $\phi$.

Theorem 2. Suppose Assumptions 1-3 are satisfied. Then,

(a) a full equilibrium exists;

(b) the set of full equilibria has a greatest element with respect to the set inclusion order;

(c) aggregate output in the greatest full equilibrium is higher than that of all other full equilibria.

While the full equilibrium is not necessarily unique, part (b) of the theorem shows that a greatest full equilibrium always exists-meaning that the set of active firms in such an equilibrium is a superset of the set of active firms in all other full equilibria. Put differently, there always exists a fixed point $\mathbf{G}_{\max }^{*}=\phi\left(\mathbf{G}_{\max }^{*}\right)$ of the mapping $\phi$ in (7) such that $\mathbf{G}_{\max }^{*} \supseteq \mathbf{G}^{*}$ for all fixed points $\mathbf{G}^{*}$ of $\phi$. In the remainder of the paper, we focus on the economy's greatest full equilibrium, as all failures in this equilibrium are "fundamental," in the sense that they are not driven by any type of miscoordination (for example in exit decisions). Furthermore, part (c) of Theorem 2 shows that the greatest full equilibrium also generates the greatest aggregate output (GDP) among all equilibria.

Note that the economy has a greatest full equilibrium even though firms' exit decisions are not necessarily strategic complements: the exit of an active customized firm may weaken the incentives of some or all other customized firms to exit. The absence of strategic complementarities in exit decisions is due to a type of aggregate demand externality that arises in general equilibrium with exits. The exit of a firm that makes negative profits increases household wealth and hence the demand for other goods, which in turn can weaken the incentive of the remaining firms to exit by increasing their profits. ${ }^{18}$ This means that Theorem 2 cannot be obtained from a direct application

\footnotetext{
${ }^{17}$ However, due to the presence of fixed costs, the equilibrium network of active firms $\mathbf{G}^{*}$ itself depends on the demand side of the economy. We discuss this relationship in the next subsection.

${ }^{18}$ Equivalently, this implies that mapping $\phi$ in equation (7) is not monotone, in the sense that $\mathbf{G}_{1} \subseteq \mathbf{G}_{2} \nRightarrow \phi\left(\mathbf{G}_{1}\right) \subseteq \phi\left(\mathbf{G}_{2}\right)$.
} 
of Tasrki's fixed point theorem (Topkis, 1998). Rather, we establish Theorem 2 using a new fixed point theorem in Appendix A that only requires strategic complementarities in exit decisions among profitable firms. This theorem may be of use in other settings with entry and exit and/or aggregate demand externalities. ${ }^{19}$

We conclude this discussion by a simple but important observation on the structure of the equilibrium production network. We say a customized or final good producer $i$ is downstream to firm $j$ (and $j$ is upstream to $i$ ) in the network of technological compatibilities $\mathcal{G}$ if $j$ is a direct or indirect supplier of $i$ in $\mathcal{G}$. We also say $i$ and $j$ have a horizontal relationship if they share a common (direct or indirect) downstream customer but are neither upstream or downstream to one another. ${ }^{20}$ With this terminology, an immediate consequence of Assumption 1 is that there will be upstream propagation of failures: a negative shock that leads to $i$ 's failure results in the failure of all of $i$ 's direct and indirect customized suppliers. Intuitively, a customized producer's only source of revenue is payments from its unique customer in $\mathcal{G}$. As a result, the customer's failure implies that $i$ cannot meet its fixed cost of operation either. With the same reasoning, all firms that are upstream to $i$ fail as well.

\subsection{Comparative Statics with Exogenous Production Networks}

In this subsection, we present comparative statics holding the set of active firms (and hence, the economy's production network) fixed at some $\mathbf{G} \subseteq \mathcal{G}$. Since the bargaining equilibrium is unique, the comparative statics in this subsection refer to changes in this unique equilibrium. We consider comparative statics with an endogenous $\mathbf{G}^{*}$ in the next subsection.

Our first result characterizes how markups, prices, and profits depend on bargaining powers.

Theorem 3. Consider a feasible production network of active firms $\mathbf{G}$. For $(j, i) \in \mathbf{G}$, an increase in $\delta_{i j}$

(a) increases the markups of $j$ and all firms upstream to $j$ and decreases the markups of all other firms;

(b) increases the profits of $j$ and all firms upstream to $j$ and decreases the profits of all other firms;

(c) increases the prices of $j$ and all firms upstream and downstream to $j$ and decreases the price of all other firms.

This theorem gives a first glimpse of the complex interactions between supply chains and equilibrium prices. Intuitively, an increase in the bargaining power of a firm vis-à-vis its customer translates into (weakly) higher prices, profits, and markups not only for that firm, but for all its direct and indirect customized suppliers as well. This result is a consequence of how gains from trade are shared between parties in pairwise bargaining. As we discussed in the context of equations (5) and (6), an increase in $j$ 's bargaining power vis-à-vis its customer $i$ allows $j$ to charge a higher price, increasing the share of the surplus that it captures. But since $j$ 's input price is itself determined via pairwise

\footnotetext{
${ }^{19}$ A second potential source of strategic substitutability in firms' exit decisions is competition for customers or suppliers. For example, the exit of firm $i$ can increase $j$ 's profits if they compete for the same set of customers or suppliers. These strategic substitutabilities are ruled out by Assumption 1, which ensures that customized firms only compete with the competitive fringe of generic producers and enables us to apply our fixed point theorem in Appendix A.

${ }^{20}$ Since $\mathcal{G}$ contains no cycles (Assumption 1), the sets of firms that are upstream, downstream, and horizontal to any given firm $j$ are mutually exclusive.
} 
bargaining with its own (customized) suppliers, $j$ 's higher surplus is partially shared with its suppliers (via higher input prices for $j$ ). This increases the markups and profits of $j$ 's suppliers. A similar logic implies that a higher $\delta_{i j}$ results in weakly higher markups, profits, and prices for all firms that are upstream to $j$. However, the same increase in $\delta_{i j}$ reduces the equilibrium markup and profit of firm $i$ and hence the markups and profits of all other firms that are either horizontal or downstream to $j$.

Finally, part (c) of Theorem 3 shows that downstream and horizontal prices move in opposite directions in response to an increase in $\delta_{i j}$. This is because for the markups of all firms that are either downstream or horizontal to $j$ to decrease simultaneously, the former group needs to charge a higher price while those in the latter group charge a lower price.

Our next result provides comparative statics with respect to the production network structure. To express this result, let $\operatorname{GDP}(\mathbf{G})$ denote aggregate output under production network $\mathbf{G}$. Similarly, let $\mu_{i}(\mathbf{G})$ and $\pi_{i}(\mathbf{G})$ denote, respectively, firm $i$ 's markup and profit (gross of fixed costs) under $\mathbf{G}$.

Theorem 4. Let $\underline{\mathbf{G}}$ and $\overline{\mathbf{G}}$ denote two feasible production networks such that $\underline{\mathbf{G}} \subseteq \overline{\mathbf{G}}$. Then,

(a) $\mu_{i}(\underline{\mathbf{G}}) \leq \mu_{i}(\overline{\mathbf{G}})$ for all $i \in \underline{\mathbf{G}}$;

(b) if

$$
\sum_{j \in \overline{\mathbf{G}} \backslash \underline{\mathbf{G}}}\left(\pi_{j}(\overline{\mathbf{G}})-w z_{j}\right) \geq 0
$$

then $\operatorname{GDP}(\underline{\mathbf{G}}) \leq \operatorname{GDP}(\overline{\mathbf{G}})$;

(c) if condition (8) is satisfied, then $\pi_{i}(\underline{\mathbf{G}}) \leq \pi_{i}(\overline{\mathbf{G}})$ for all $i \in \underline{\mathbf{G}}$.

Statement (a) establishes that more exits lead to weakly lower markups throughout the economy. This result is intuitive: since customized producers are more productive than their generic counterparts (Assumption 2), the presence of an additional customized producer increases the surplus it can split with its customer, which is then shared with other firms via pairwise bargaining.

The last two parts of the theorem establish that exit of profitable firms reduces aggregate output and the profits of all remaining firms. Intuitively, with fewer customized firms, production relies more on generic inputs and as a result, the (aggregate) production possibility frontier shifts in. The reason we need to focus on exit of profitable firms_imposing inequality (8)—is that removing loss-making firms would increase the income of the representative household and aggregate demand, creating countervailing effects. ${ }^{21}$ This result will play an important role when we turn to the comparative statics of the economy's full equilibrium, as it ensures that exit decisions reinforce the effects of changes in productivity and/or fixed costs that decrease firm profitability.

Our final result of this subsection establishes comparative static results with respect to productivity shocks.

Theorem 5. Consider a feasible production network of active firms $\mathbf{G}$. For $(j, i) \in \mathbf{G}$, an increase in $A_{i j}$ (a) increases all markups in the economy;

\footnotetext{
${ }^{21}$ Recall from footnote 19 that firms' exit decisions may be strategic substitutes due to this aggregate demand externality. However, statement (c) of the theorem establishes that exit decisions of profitable firms are always strategic complements.
} 
(b) increases aggregate output;

(c) increases the profits of $i$ and $j$, and those of all firms that are either downstream or horizontal to $j$;

(d) furthermore, if $\delta_{j r}=1$ for all suppliers $r$ of $j$, an increase in $A_{i j}$ decreases the profits of all firms that are upstream to $j$.

An increase in the relationship-specific productivity of a pair of customized firms translates into higher aggregate output, with the corresponding surplus shared with other firms in the economy in the form of higher markups. The intuition for this result, which is a consequence of the noncompetitive nature of the economy, is familiar from our discussion of Theorem 4. More novel is the non-monotonic impact on profits. While firms that are downstream to $j$ increase their profits as a result of the higher productivity, $j$ 's suppliers may lose out because of a "perverse quantity effect": the increase in productivity means that $i$ 's demand for $j$ 's input declines, and though this can never reduce $j$ 's profitability, it can push down the profits of $j$ 's direct and indirect suppliers. We will see in the next subsection that this negative impact of higher productivity on supplier profits can translate into non-monotonic effects on aggregate output when the production network is endogenous.

\subsection{Comparative Statics with Endogenous Production Networks}

We now turn to our main theoretical results, which characterize the macroeconomic impact of shocks when production networks are endogenous. The next theorem characterizes the implications of changes in fixed costs.

Theorem 6. In the greatest full equilibrium, an increase in fixed costs of operation

(a) shrinks the set of active firms;

(b) lowers aggregate output;

(c) reduces markups and profits of all surviving firms.

A higher fixed cost of operation for a firm, say firm $i$, makes it more likely to fail. More interestingly, it makes all other firms in the economy more likely to exit as well. This is because of both partial and general equilibrium effects. The partial equilibrium effect, which is the central mechanism of our model, is due to the propagation of the shock over the production network: firm $i$ 's exit makes its (direct and indirect) customers and suppliers and firms that are horizontal to it less profitable, and hence more likely to exit. In addition, as previously profitable firms fail, household wealth and thus demand for all products is reduced. This aggregate demand externality further amplifies the impact of the initial negative shock and may contribute to additional failures.

Changes in productivity have more complex implications than changes in fixed costs because of the perverse quantity effect we saw in Theorem 5. To abstract from this effect, we first focus on a network in which the customized firm experiencing higher productivity has no active suppliers.

Theorem 7. Let $\mathbf{G}^{*}$ denote the economy's greatest full equilibrium and let $(j, i) \in \mathbf{G}^{*}$ be a suppliercustomer pair of active firms. If $j$ does not have any active suppliers in $\mathbf{G}^{*}$, then an increase in $A_{i j}$ 
(a) expands the set of active firms;

(b) increases all active firms' profits;

(c) increases aggregate output.

This theorem shows that when a customized firm with no active suppliers enjoys a higher productivity, profits of all firms increase, exit of other firms becomes less likely, and aggregate output increases. Intuitively, we know from Theorem 5 that an increase in productivity of a customized firm, say $j$, raises the profits of all firms except those that are upstream to it. When firm $j$ has no suppliers, all firms in the economy benefit from its higher productivity. Then the partial and general equilibrium effects discussed above both contribute to raising the profits of all other firms, making their exit less likely and increasing aggregate output. However, when firm $j$ has suppliers, the perverse quantity effect from part (d) of Theorem 5 reemerges and these results may no longer hold. This is illustrated in the next example and analyzed more systematically in our final theorem in this subsection.

Example 1. Consider an economy consisting of five customized firms in five separate industries, labeled $\{1,2,3,4,5\}$, and a single firm producing the consumption good. Figure 3 depicts the underlying network of technological compatibilities, $\mathcal{G}$. For simplicity, we assume that the supplier firm has all the bargaining power in all pairwise relationships, firm 4 is the only firm with a non-zero fixed cost (i.e., $z_{i}=0$ for all $i \neq 4$ ), and firms 2 and 5 are the only firms that use labor as an input. Furthermore, we assume that productivities $\left(B_{12}, B_{13}, B_{34}, B_{45}\right)$ and $\left(A_{12}, A_{13}, A_{34}, A_{45}\right)$ are such that $\mathbf{G}^{*}=\mathcal{G}$, that is, all firms are active in the economy's greatest equilibrium.

In such an economy, the aggregate output and the profit of firm 4, gross of fixed costs, are given by

$$
\begin{aligned}
\mathrm{GDP} & =\left(A_{12}^{-1}+A_{13}^{-1} A_{34}^{-1} A_{45}^{-1}\right)^{-1}\left(L-z_{4}\right) \\
\pi_{4} & =w\left(B_{34}^{-1}-A_{34}^{-1}\right) \mathrm{GDP} /\left(A_{13} B_{45}\right),
\end{aligned}
$$

where $L$ denotes the aggregate supply of labor.

A few observations are immediate. First, as shown in Theorem 5, when the production network is held fixed, aggregate output is increasing in all customized productivities. Second, $\pi_{4}$ is decreasing in $A_{13}$, as predicted by Theorem 5(d): the aforementioned perverse quantity effect implies that higher downstream productivity decreases firm 4's profits because of the reduction in demand from firm 3. Nonetheless, as long as the economy's production network is held fixed, a higher $A_{13}$ raises aggregate output.

Next, consider the case in which we allow the equilibrium production network to respond to an increase in productivity from $A_{13}$ to $\bar{A}_{13}$. Since firm 4's profit is decreasing in $A_{13}$, this increase in productivity may result in 4's failure, which in turn makes firm 5 unable to produce as well. As a consequence, aggregate output after the productivity increase is given by

$$
\operatorname{GDP}\left(\bar{A}_{13}\right)=\left(A_{12}^{-1}+\bar{A}_{13}^{-1} B_{34}^{-1} B_{45}^{-1}\right)^{-1} L .
$$

Comparing equations (9) and (10) shows that, for small enough values of $z_{4}$, and as long as $\bar{A}_{13} B_{34} B_{45}<A_{13} A_{34} A_{45}$, the increase in productivity reduces aggregate output. 


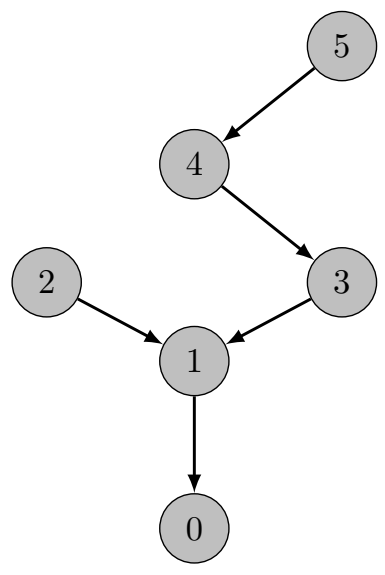

Figure 3. An economy consisting of five customized producers in five different industries and one final good producer. The figure depicts the firm-level network $\mathcal{G}$ of technological compatibilities. For simplicity, the figure does not include generic producers.

Theorem 8. Let $\overline{\mathbf{G}}^{*}$ denote the greatest full equilibrium under productivity $\bar{A}_{i j}$ for supplier-customer pair of firms $(j, i)$ and suppose there is a supplier-customer sequence of firms $\left(k_{0}, k_{1}, \ldots, k_{s}, j\right)$ upstream to $j$ that only use a single input, with $k_{1} \notin \overline{\mathbf{G}}^{*}$ and $\delta_{k_{2} k_{1}}=1$. Then, for any $\underline{A}_{i j}<\bar{A}_{i j}$ such that $\pi_{k}\left(\overline{\mathbf{G}}^{*}, \underline{A}_{i j}\right) \geq w z_{k}$ for all $k \in \overline{\mathbf{G}}^{*}$, there exists $\theta<1$, such that if $\pi_{k_{1}}\left(\overline{\mathbf{G}}^{*} \cup\left\{k_{1}\right\}, \bar{A}_{i j}\right)>\theta w z_{k_{1}}$, then

(a) $\overline{\mathbf{G}}^{*} \subsetneq \underline{\mathbf{G}}^{*}$, where $\underline{\mathbf{G}}^{*}$ is the full equilibrium under $\underline{A}_{i j}$.

(b) Furthermore, there exist $\tilde{A}_{k_{1} k_{0}}$ and $\tilde{z}_{k}$ such that if $A_{k_{1} k_{0}}>\tilde{A}_{k_{1} k_{0}}$ and $z_{k_{0}}<\tilde{z}_{k_{0}}$, then $\operatorname{GDP}\left(\overline{\mathbf{G}}^{*}, \bar{A}_{i j}\right)<$ $\operatorname{GDP}\left(\underline{\mathbf{G}}^{*}, \underline{A}_{i j}\right)$.

The intuition for why productivity improvements may be recessionary comes from part (d) of Theorem 5 and Example 1: higher productivity of firm $j$ reduces the profitability of its suppliers via the perverse quantity effect. In Theorem 5 , this non-monotonic effect did not reverse the initial positive impact of the initial productivity increase on aggregate output. In contrast, Theorem 8 establishes that, because of the endogenous response of the production network, a higher $A_{i j}$ may push $j$ 's suppliers into failure, resulting in a supply chain disruption. This partial equilibrium propagation is further magnified with the general equilibrium effects working through reduced household wealth and aggregate demand for other products.

To be sure, we do not view the situation identified in Theorem 8 to be a major source of downturns in practice. Nevertheless, together with Theorem 7, this result illustrates how the propagation of (potentially small) shocks over an endogenous production network can lead to rich economic dynamics. The former result explains how a negative shock can be amplified due to extensive margin adjustments, whereas the latter illustrates how a positive shock can be recessionary under some circumstances-in particular, when it leads to the failure of sufficiently productive upstream suppliers. We next turn to a more systematic decomposition of the effects of shocks on aggregate output between exogenous and endogenous supply chain components. 


\subsection{Aggregate Implications}

We end this section with a formal decomposition of the aggregate impact of shocks into two components-one that holds the production network fixed and one that traces the consequence of the endogenous response of the production network. For brevity, we present this decomposition for a change in fixed costs of operation and provide the analogous result for changes in productivities in Appendix D.

Theorem 9. Let $\bar{z}_{i} \geq \underline{z}_{i}$ for all $i$. The change in aggregate output in the economy's greatest full equilibrium in response to the decrease in fixed costs from $\bar{z}$ to $\underline{z}$ is

$$
\Delta \mathrm{GDP}=\left.\Delta \mathrm{GDP}\right|_{\overline{\mathrm{G}}^{*} \text { fixed }}+\left.\Delta \mathrm{GDP}\right|_{\underline{z} \text { fixed }},
$$

where $\overline{\mathbf{G}}^{*}$ and $\underline{\mathrm{G}}^{*}$ are the production networks in the greatest full equilibria under $\bar{z}$ and $\underline{z}$ and

$$
\begin{aligned}
\left.\Delta \mathrm{GDP}\right|_{\overline{\mathbf{G}}^{*} \text { fixed }} & =\operatorname{GDP}\left(\overline{\mathbf{G}}^{*}, \underline{z}\right)-\operatorname{GDP}\left(\overline{\mathbf{G}}^{*}, \bar{z}\right) \geq 0 \\
\Delta \mathrm{GDP} & \left.\right|_{\underline{z} \text { fixed }}=\operatorname{GDP}\left(\underline{\mathbf{G}}^{*}, \underline{z}\right)-\operatorname{GDP}\left(\overline{\mathbf{G}}^{*}, \underline{z}\right) \geq 0 .
\end{aligned}
$$

This theorem establishes that the direct impact of the shock (the first term on the right-hand side of (11)) is amplified by the endogenous adjustment of the production network due to firm failures (the second term in (11), which has the same sign as the first term). ${ }^{22}$

The next example shows that long supply chains can be a source of fragility and illustrates how this fragility can render the indirect effects working via the response of the equilibrium production network much larger than a shock's direct impact.

Example 2. Consider an economy consisting of $n$ customized firms indexed $\{1, \ldots, n\}$ in $n$ separate industries and a single final good producer 0 , with the network of technological compatibilities $\mathcal{G}$ depicted in Figure 4. All firms use a single input for production. For simplicity, we assume that all pairwise relationships are identical in terms of productivities and bargaining power, i.e., $A_{i, i+1}=A$, $B_{i, i+1}=B$, and $\delta_{i, i+1}=\delta$ for all $i$, where $A>B \geq 1$ and $\delta>1 / 2$. This latter assumption means that, in any pairwise relationship, the supplier has a greater bargaining power than the customer. Finally, suppose that firm 1 is the only customized firm with a non-zero fixed cost (i.e., $z_{i}=0$ for all $i \neq 1$ ). This means that, in any equilibrium, either all $n$ customized firms operate or they all fail.

Let $\tilde{z}_{1}$ denote the threshold on firm 1's fixed cost above which the firm fails. Since 1's failure results in the failure of all other customized producers, aggregate output in this economy is given by

$$
\mathrm{GDP}= \begin{cases}A^{n-1}\left(L-z_{1}\right) & \text { if } z_{1} \leq \tilde{z}_{1} \\ B^{n-1} L & \text { if } z_{1}>\tilde{z}_{1} .\end{cases}
$$

Moreover, as long as outside options in pairwise bargains do not bind, the failure threshold on firm 1's fixed cost can be characterized as

$$
\tilde{z}_{1}=\frac{1-\delta}{2 \delta-1}\left(1+\frac{\left(\frac{\delta}{1-\delta}\right)^{n}-1}{(A / B)^{n-1}-1}\right)^{-1} L .
$$

\footnotetext{
${ }^{22}$ Because we are considering arbitrary (rather than infinitesimal) shocks, the order of the decomposition in Theorem 9 matters. The one we choose looks at the adjustment of GDP that would have happened holding the production network constant at its value in the larger network and then considers the additional impact of having fewer active firms.
} 


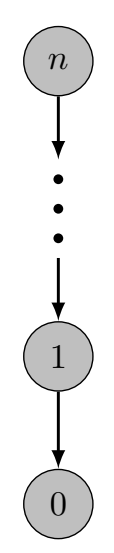

Figure 4. Production Chain

Equations (12) and (13) have two important implications. On the one hand, equation (12) implies that, when $z_{1} \leq \tilde{z}_{1}$, aggregate output is increasing in the number of firms, $n$, in the production chain. This is because a longer chain shifts out the production possibility frontier. On the other hand, equation (13) illustrates that, if $(1-\delta) A<\delta B$, the threshold $\tilde{z}_{1}$ decreases in $n$, making it more likely for all firms to fail in a longer the chain. In other words, despite the increase in the economy's productive capacity, a longer production chain is more fragile in response to shocks. This disparity is driven by the non-competitive nature of how surplus from this increased productivity is shared between various firms: the greater surplus from the longer chain is shared among more firms, leading to a smaller surplus for firm 1.

We can also use equations (12) and (13) to obtain a decomposition of the direct and indirect impact of a shock on aggregate output similar to Theorem 9. For example, consider an increase in firm 1's fixed cost from $\underline{z}_{1}=\tilde{z}_{1}-\varepsilon / A^{n-1}$ to $\bar{z}_{1}=\tilde{z}_{1}+\varepsilon / A^{n-1}$ for some small $\varepsilon>0$. Then, holding the production network of the economy fixed, the shock has a direct impact on GDP of $-2 \varepsilon$. However, this shock also pushes firm 1, and hence the rest of the customized firms, into the failure region. From equation (12), the indirect effect of the shock due to firm failures thus reduces aggregate output by $A^{n-1}(L-\tilde{z})-$ $B^{n-1} L-\varepsilon$. This latter effect can be arbitrarily large relative to the direct effect when the supply chain is long ( $n$ large) or when the shock that triggers the cascades of failures is small ( $\varepsilon$ small).

\section{Extensions}

In this section, we discuss two extensions. First, we relax the assumption of Leontief production technologies and show that our results in Section 4 extend to a broad class of economies where inputs are gross complements. Second, we briefly discuss how the framework can be extended to incorporate entry and matching decisions.

\subsection{General Production Technologies}

We start by defining a few important concepts. We say that industry J's input is essential for the production technology of industry $I$ if (i) the marginal cost $C_{I}$ is strictly positive whenever the price of 
input $J$ is strictly positive and (ii) $C_{I}$ is strictly increasing in input $J$ 's price whenever all input prices are non-zero. Similarly, we say $J$ is an indirect essential input for the production technology of $I$, if there exits a sequence of industries starting with $J$ and terminating at $I$ such that each input in the sequence is essential for the production technology of the next. We can use parallel notions to define labor as a direct or indirect essential input.

Let $\sigma_{i}^{k j}$ denote the Morishima elasticity of substitution between distinct pair of inputs (including labor) $k$ and $j$ in the production technology of any given firm $i .^{23}$ We replace Assumption 3 with the following restriction on firms' production technologies.

Assumption $3^{\prime}$. The collection of cost functions $\left(C_{1}, \ldots, C_{n}\right)$ satisfy the following:

(i) labor is a direct or indirect essential input for the technology of all industries;

(ii) $\sigma_{i}^{k j} \leq 1$ for all firms $i$ and all pairs of inputs $k \neq j$;

(iii) for all pairs of inputs $k \neq j$,

$$
\frac{\mathrm{d} \sigma_{i}^{k j}}{\mathrm{~d} \log p_{i j}} \geq-\left(1-\sigma_{i}^{k j}\right)^{2} .
$$

The first condition imposed by Assumption $3^{\prime}$ rules out scenarios in which labor is an inessential input for production of any given industry, thus ensuring that aggregate output is always finite. The second condition significantly relaxes Assumption 3 by simply requiring that inputs from different industries are gross complements in all production functions. ${ }^{24}$ Finally, the third condition imposes a bound on how quickly these elasticities change in response to changes in input prices. Any CES production technology, for which these elasticities remain constant, trivially satisfies inequality (14).

The next theorem establishes the existence and uniqueness of production equilibria as well as existence of bargaining equilibria under Assumption $3^{\prime}$.

Theorem 10. Suppose Assumptions 1, 2, and $3^{\prime}$ are satisfied. For any feasible network of active firms $\mathbf{G}$,

(a) a production equilibrium always exists and is unique;

(b) a bargaining equilibrium $\left(\boldsymbol{p}^{a}, \boldsymbol{p}^{b}\right)$ always exists. Furthermore, in any bargaining equilibrium, all pairs of firms $(j, i) \in \mathbf{G}$ reach an agreement;

(c) the vector of generic prices $\boldsymbol{p}^{b}=\left(p_{1}^{b}, \ldots, p_{n}^{b}, w\right)$ is unique (up to a scaling) and is the solution to the system of equations

$$
p_{i}^{b}=C_{I}\left(w, p_{1}^{b} / B_{i 1}, \ldots, p_{n}^{b} / B_{i n}\right)
$$

(d) the vector of customized prices $\boldsymbol{p}^{a}=\left(p_{i j}^{a}\right)_{(j, i) \in \mathbf{G}}$ is the solution to the system of equations

$$
p_{i j}^{a}=\min \left\{\hat{p}_{i j}, p_{j}^{b} A_{i j} / B_{i j}\right\},
$$

\footnotetext{
${ }^{23}$ The Morishima elasticity of substitution between inputs $k$ and $j$ is defined as $\sigma_{i}^{k j}=\epsilon_{i}^{k j}-\epsilon_{i}^{j j}$, where $\epsilon_{i}^{k j}$ is firm $i$ 's constantoutput elasticity of demand for input $k$ with respect to changes in the price of input $j$.

${ }^{24}$ However, recall that (3) implies that the generic and customized variants produced by any given industry are always perfect substitutes.
} 
where $\hat{p}_{i j}$ is the unique solution to equation

$$
f_{i j}\left(\hat{p}_{i j}\right)=\delta_{i j} \pi_{i} \frac{\partial \pi_{j}}{\partial p_{i j}^{a}}+\left(1-\delta_{i j}\right) \pi_{j} \frac{\partial \pi_{i}}{\partial p_{i j}^{a}}=0
$$

where $\pi_{i}$ and $\pi_{j}$ denote the profits of firms $i$ and $j$ gross of fixed operating costs.

This theorem is a direct generalization of Theorem 1, with the only significant difference being in the way markups and prices are determined: generic and customized prices are now given by the system of equations (15)-(17). This disparity is a consequence of the fact that the nature of input complementarities alters the strategic interactions in pairwise bargaining games. To see the intuition for equation (17), recall that in their pairwise bargaining game, supplier $j$ and customer $i$ can make offers with probabilities $\delta_{i j}$ and $1-\delta_{i j}$, respectively. As a result, when the outside options do not bind (and taking all other prices as given), a pair of prices $p_{i j}^{\prime}$ and $p_{i j}^{\prime \prime}$ corresponds to a SPNE of the pairwise bargaining game if and if they satisfy the following indifference conditions for $j$ and $i$,

$$
\begin{aligned}
& \pi_{j}\left(p_{i j}^{\prime}\right)=\eta \delta_{i j} \pi_{j}\left(p_{i j}^{\prime \prime}\right)+\eta\left(1-\delta_{i j}\right) \pi_{j}\left(p_{i j}^{\prime}\right) \\
& \pi_{i}\left(p_{i j}^{\prime \prime}\right)=\eta \delta_{i j} \pi_{i}\left(p_{i j}^{\prime \prime}\right)+\eta\left(1-\delta_{i j}\right) \pi_{i}\left(p_{i j}^{\prime}\right),
\end{aligned}
$$

respectively, where $\eta$ is the firms' common discount factor. ${ }^{25}$ This system of equations reduces to (17) as $\eta \rightarrow 1$ and the equilibrium offers satisfy $\lim _{\eta \rightarrow 1} p_{i j}^{\prime}=\lim _{\eta \rightarrow 1} p_{i j}^{\prime \prime}=\hat{p}_{i j}$, where $\hat{p}_{i j}$ is the unique solution to equation (17). This observation further illustrates that the non-linearity in equation (17) is because the pairwise bargaining game between the two firms is a game of non-transferable utility: the two parties bargain over fixed-price contracts, even though their pairwise surplus and their payoffs are non-linear in the agreed price. ${ }^{26}$ In the special case where the customer's production function is Leontief, equation (17) reduces to equation (5) in Theorem 1.

We next show that all of our comparative static results from Theorems 2-8 generalize to economies with complementarities between inputs provided that Assumption $3^{\prime}$ is satisfied. For tractability, we also restrict attention to the special case in which $\delta_{i j}=1$ for all $(j, i) \in \mathbf{G}$-that is, all bargaining power is vested in suppliers. The economics of these comparative statics is identical to those in the economy with Leontief technologies, though the quantitative effects differ depending on the degree of complementarity between various inputs.

Theorem 11. Suppose Assumptions 1, 2, and $3^{\prime}$ hold. Furthermore, suppose that $\delta_{i j}=1$ for all $(j, i) \in \mathcal{G}$. Then, Theorems 2 and 4-8 apply.

\subsection{Entry and Matching}

Our analysis thus far focused on the implications of negative shocks-modeled via comparative statics-on exits and aggregate output, taking network of technological compatibilities $\mathcal{G}$ between

\footnotetext{
${ }^{25}$ Note that we do not restrict the firms' strategies to be stationary, as each firm's offers and reactions to offers may depend on the entire history of actions. Nonetheless, as in Rubinstein (1982), the unique SPNE of the pairwise bargaining game turns out to be stationary.

${ }^{26}$ This non-linearity may also lead to a non-convex payoff set in the bargaining game, which may then result in multiple SPNEs. However, even when the set of payoffs is non-convex, the curvature restrictions imposed in part (iii) of Assumption $3^{\prime}$ guarantee that the game between $i$ and $j$ has a unique SPNE and is given by the unique solution to equation (17).
} 
various customized firms as given. This raises two related questions: (i) where does $\mathcal{G}$ comes from and how does it adjust in the face of shocks? And (ii) in what sense can increases in fixed costs or reductions in productivity be viewed as "negative shocks" that hit the economy at business cycle frequencies?

While a systematic analysis of these questions is beyond the scope of this paper, in this subsection we provide a brief outline of how the model can be generalized to speak to these issues.

Consider an extension of the model with two more time periods, $t \in\{-2,-1\}$. At $t=-2$, a large set of potential entrants decide to enter as customized producers into any one of the sectors of the economy by paying a fixed cost of $\chi_{J}$ for sector $J$ (there is no costly entry stage for generic producers, since they always make zero profits in equilibrium). Upon entry at $t=-1$, customized producer $j$ in industry $J$ decides whether to pay another fixed cost, denoted by $\gamma_{j}$, to match randomly with potential suppliers and customers. This is meant to capture the costs involved in developing trust, reputation, and the requisite knowledge for long-term customized relationships. The outcome of this random matching process is the network of technological compatibilities $\mathcal{G}$. When making their entry and matching decisions at $t=-2$ and $t=-1$, respectively, firms face incomplete information about the outcome of the matching process, productivities, and fixed costs of operation $z_{j}$, all of which are revealed at $t=0$. Free entry thus requires that, for each industry $J$, the entry of one more firm would lead to

$$
\mathbb{E}_{-2}\left[\max \left\{\mathbb{E}_{-1} \max \left\{\pi_{j}\left(\overline{\mathbf{G}}^{*}\right)-w z_{j}, 0\right\}-\gamma_{j}, 0\right\}\right]-\chi_{J}<0,
$$

while this expression is nonnegative for the firms that enter in equilibrium. The inner max operator captures the fact that after the matching pattern, productivities, and fixed costs of operation are revealed at $t=0$, the firm would exit if it is more profitable to do so, in which case it has a zero continuation profit (though it has already incurred the entry and matching fixed costs). The outer max operator recognizes that after entry at $t=-2$, firms can still decide not to pay the fixed cost of matching. Finally, the expectation operator $\mathbb{E}_{t}$ incorporates the information available to firms at time $t$ about the outcome of the matching process, productivities, and fixed costs of operation. In general, $\mathbb{E}_{-1}$ may be different from $\mathbb{E}_{-2}$, allowing for additional information to be revealed to firms between the entry and matching stages.

This formalization is useful for clarifying a few points. First, recall that our comparative statics are carried out under complete information at time $t=0$, but they can be thought of as being due to "realizations of negative shocks" from the viewpoint of $t=-2$ or -1 . Second, firm exit at time $t=0$ is qualitatively different from firm entry, which occurs at earlier points on the basis of expectations of productivities, fixed costs, and matching patterns.

Third, this formalism enables us to discuss different types of shocks and responses thereto. The first type of shock is the one we have discussed so far, taking place at $t=0$. These should be thought of as shocks that happen after medium-term entry and customization/matching decisions have been made. At this point, there is no further adjustment in the economy's production network except for the exit decisions. In contrast, there can be other margins of adjustments in response to a time $t=-1$ negative shock, which may reveal that some firms will end up with a higher fixed costs than expected, some suppliers have low productivity, or certain matches may not form. For example, if the fixed costs of operation for a set of firms in an industry turn out to be high, so that they will not take part in 
matching or drop out with a high likelihood after matching, then other firms may make up this slack by paying the cost for matching and forming new relationships. Conversely, new information that arrives at this point may discourage the formation of customized relationships. This highlights that negative shocks at $t=-1$ may generate dampening or amplifying responses through the matching process. Finally, shocks that arrive at time $t=-2$ may increase or reduce entry. We think of these last two types of responses as taking place in the medium run (say, within horizon of a few years), while what we focused on in the rest of the paper corresponds to shorter-run responses (say, a horizon of a few quarters). The full analysis of comparative statics in this extended model is an area for future research.

\section{Conclusions}

Most modern economies rely on complex supply chains, where multiple layers of suppliers enter into specific, customized relationships with one other. This type of customization often enables greater efficiency in production, but may also create fragilities as relatively small shocks can result in significant supply chain disruptions.

A key contribution of our paper is to develop a novel and tractable (non-competitive) model of the distribution of relationship-specific surplus over supply chains. Our paper studies how this distribution is shaped by the structure of the production network and differences in productivities, and how it regulates and amplifies the response of the macroeconomy to recessionary shocks.

Our analysis shows that changes in productivity alter the distribution of surplus throughout the production network and determine which firms are at the margin of failure. Via a number of general comparative statics theorems, we demonstrate that a negative shock propagates in the production network as a firm's failure reduces the profits of its suppliers, customers, and possibly other firms in the economy, bringing them to the brink of failure as well. At the root of these results is a new fixed point theorem, which enables us to show that, although our economy does not feature strategic complementarities, exit of a previously profitable firm tends to cascade into additional exits.

Lack of general strategic complementarities can also be seen from the fact that even positive shocks may be recessionary. This is because of the non-monotonic impact of productivity increases, which may reduce demand for upstream firms and force their exit. These exits, and the costs associated with the disruption of productive supply chains, can outweigh the direct positive impact of the initial shock.

Our general comparative statics are established under some key simplifying assumptions, however. Most importantly, we assume that each customized firm can source each input from a single customized supplier (though may have multiple suppliers if it uses multiple inputs) and each customized firm can only have a single customized customer. We also abstract from relationshipspecific investments that increase the efficiency of customized relationships, the formation of supplier-customer matches, and the substitution of new suppliers for those that fail. These constitute important and challenging areas for future theoretical analysis.

An even more important direction for future research would be a systematic analysis of the empirics of supply chains in economic fluctuations. A first step would be a detailed quantitative 
evaluation of the amplification generated by supply chain disruptions. While recent work, including Jacobson and von Schedvin (2015) and Carvalho et al. (2017), documents the propagation of shocks over firm-level supply chains, there is still much that we do not know about the role of supply chains in the macroeconomy. These include: how prices and quantities in supply chains vary over the business cycle, how supply chains become disrupted and repaired following negative shocks, and where the most major systemic supply chain fragilities lie. Such analyses could be relevant for policy as well, since, as the recent global economic crisis triggered by the COVID-19 pandemic illustrates, the collapse of national and global supply chains can be a critical threat to the economy.

\section{Appendix}

\section{A A Set-Valued Fixed Point Theorem}

This appendix states and proves a new fixed point theorem for set-valued mappings. We apply this result in the subsequent proofs to establish the existence of the economy's full equilibrium, show that the set of full equilibria has a greatest element, and obtain comparative static results when the economy's production network is endogenous. This fixed point theorem may be of more general use in economies with entry and exit and/or aggregate demand externalities.

Let $\mathcal{S}$ denote a finite set and $\phi: 2^{\mathcal{S}} \rightarrow 2^{\mathcal{S}}$ denote a mapping from the subsets of $\mathcal{S}$ to itself.

Assumption A.1 (weak monotonicity). For any $S_{1}, S_{2} \subseteq \mathcal{S}$, if $S_{1} \subseteq \phi\left(S_{2}\right)$, then $S_{1} \subseteq \phi\left(S_{1} \cup S_{2}\right)$.

Assumption A.2 (weak superadditivity). If $S_{1} \subseteq \phi\left(S_{1}\right)$ and $S_{2} \subseteq \phi\left(S_{2}\right)$, then $S_{1} \cup S_{2} \subseteq \phi\left(S_{1} \cup S_{2}\right)$.

It is easy to verify that Assumptions A.1 and A.2 are weaker than monotonicity with respect to the set inclusion order, which requires that $\phi\left(S_{1}\right) \subseteq \phi\left(S_{2}\right)$ whenever $S_{1} \subseteq S_{2}$. We have the following result:

Theorem A.1. Suppose mapping $\phi: 2^{\mathcal{S}} \rightarrow 2^{\mathcal{S}}$ satisfies Assumptions A.1 and A.2. Then,

(a) $\phi$ has a fixed point, i.e., there exists $S^{*} \subseteq \mathcal{S}$ such that $S^{*}=\phi\left(S^{*}\right)$;

(b) $\phi$ has a greatest fixed point, i.e., there exists $\bar{S}^{*}$ such that $\bar{S}^{*}=\phi\left(\bar{S}^{*}\right)$ and $\bar{S}^{*} \supseteq S^{*}$ for all $S^{*}=\phi\left(S^{*}\right)$;

(c) the greatest fixed point of $\phi$ is given by $\bar{S}^{*}=\bigcup_{S \in \mathcal{X}} S$, where $\mathcal{X}=\{S \subseteq \mathcal{S}: S \subseteq \phi(S)\}$.

Statements (a) and (b) of Theorem A.1 extend Tarski's fixed point theorem (which only applies to monotone mappings over a complete lattice) to non-monotone maps that satisfy the weaker requirements of Assumptions A.1 and A.2. Statement (c) then provides a characterization of $\phi$ 's greatest fixed point in terms of set $\mathcal{X}=\{S \subseteq \mathcal{S}: S \subseteq \phi(S)\}$, which will prove useful in our subsequent results. In particular, it leads to the following corollary, which generalizes Theorem 3 of Milgrom and Roberts (1994):

Corollary A.1. Suppose $\phi_{1}, \phi_{2}: 2^{\mathcal{S}} \rightarrow 2^{\mathcal{S}}$ satisfy Assumptions A.1 and A.2. If $\phi_{1}(S) \subseteq \phi_{2}(S)$ for all $S \subseteq \mathcal{S}$, then $\bar{S}_{1}^{*} \subseteq \bar{S}_{2}^{*}$, where $\bar{S}_{1}^{*}$ and $\bar{S}_{2}^{*}$ are the greatest fixed points of $\phi_{1}$ and $\phi_{2}$, respectively. 


\section{Proof of Theorem A.1}

Proof of part (a) Consider the sequence of sets $S_{k+1}=\phi\left(S_{k}\right)$ with the initial condition $S_{0}=\varnothing$. If $S_{k} \subseteq S_{k+1}$ for all $k$, then it is immediate that $S_{\infty}=\lim _{k \rightarrow \infty} \phi^{(k)}\left(S_{0}\right)=\cup_{k \geq 0} S_{k}$ exists and is a fixed point of $\phi$. It is therefore sufficient to show that $S_{k} \subseteq S_{k+1}$ for all $k$. We prove this claim by induction. The base of the induction holds trivially as $S_{0}=\varnothing$. As the induction hypothesis, suppose that $S_{k-1} \subseteq S_{k}=$ $\phi\left(S_{k-1}\right)$. Given that $S_{k} \subseteq \phi\left(S_{k-1}\right)$, Assumption A.1 implies that $S_{k} \subseteq \phi\left(S_{k} \cup S_{k-1}\right)$. But note that, by the induction hypothesis, $S_{k} \cup S_{k-1}=S_{k}$, thus implying that $S_{k} \subseteq \phi\left(S_{k}\right)=S_{k+1}$.

Proof of part (b) Define $\mathcal{X}=\{S \subseteq \mathcal{S}: S \subseteq \phi(S)\}$. The fact that $\varnothing \in \mathcal{X}$ implies that $\mathcal{X}$ is non-empty. We can therefore define $S_{0}=\bigcup_{S \in \mathcal{X}} S$ and consider the sequence $S_{k}=\phi\left(S_{k-1}\right)$. We claim that

$$
S_{k-1} \subseteq S_{k}
$$

for all $k \geq 1$. If this claim is true, then it is immediate that $\bar{S}^{*}$ defined as $\bar{S}^{*}=\lim _{k \rightarrow \infty} \phi^{(k)}\left(S_{0}\right)=$ $\bigcup_{k \geq 0} S_{k}$ exists, is a fixed point of $\phi$, and satisfies $\bar{S}^{*} \supseteq S_{0}$, where recall that $S_{0}=\bigcup_{S \in \mathcal{X}} S$. But note that $\mathcal{X}$ contains all fixed points of $\phi$. As a result, any fixed point $S^{*}=\phi\left(S^{*}\right)$ satisfies $S^{*} \subseteq S_{0} \subseteq \bar{S}^{*}$. Consequently, $\bar{S}^{*}$ is the greatest fixed point of $\phi$, containing all other fixed points as subsets.

The proof is therefore complete once we establish (A.1) for all $k \geq 1$. We do so by induction. Since set $\mathcal{X}$ is finite, Assumption A.2 guarantees that

$$
S_{0}=\bigcup_{S \in \mathcal{X}} S \subseteq \phi\left(\bigcup_{S \in \mathcal{X}} S\right)=\phi\left(S_{0}\right)=S_{1},
$$

thus establishing the base of the induction. Now, as the induction hypothesis, suppose $S_{k-1} \subseteq S_{k}$. On the other hand, since $S_{k} \subseteq \phi\left(S_{k-1}\right)$, Assumption A.1 implies that $S_{k} \subseteq \phi\left(S_{k} \cup S_{k-1}\right)$. Hence, by the induction hypothesis, $S_{k} \subseteq \phi\left(S_{k}\right)=S_{k+1}$. This establishes (A.1) and completes the proof.

Proof of part (c) Recall from the proof of part (b) that the greatest fixed point of $\phi$ is given by $\bar{S}^{*}=$ $\bigcup_{k \geq 0} S_{k}$, where $S_{k}=\phi\left(S_{k-1}\right)$ and $S_{0}=\bigcup_{S \in \mathcal{X}} S$. This immediately implies that $S_{0} \subseteq \bar{S}^{*}$. At the same time, since $\bar{S}^{*}$ is a fixed point, it also must be the case that $\bar{S}^{*} \in \mathcal{X}$, and hence, $\bar{S}^{*} \subseteq S_{0}$. Therefore, $\bar{S}^{*}=S_{0}=\bigcup_{S \in \mathcal{X}} S$.

\section{Proof of Corollary A.1}

Let $\mathcal{X}_{1}=\left\{S \subseteq \mathcal{S}: S \subseteq \phi_{1}(S)\right\}$ and $\mathcal{X}_{2}=\left\{S \subseteq \mathcal{S}: S \subseteq \phi_{2}(S)\right\}$. The assumption that $\phi_{1}(S) \subseteq \phi_{2}(S)$ for all $S \subseteq \mathcal{S}$ implies that $\mathcal{X}_{1} \subseteq \mathcal{X}_{2}$. By part (c) of Theorem A.1, the greatest fixed points of $\phi_{1}$ and $\phi_{2}$ are given by $\bar{S}_{1}^{*}=\bigcup_{S \in \mathcal{X}_{1}} S$ and $\bar{S}_{2}^{*}=\bigcup_{S \in \mathcal{X}_{2}} S$, respectively. Therefore, $\bar{S}_{1}^{*} \subseteq \bar{S}_{2}^{*}$.

\section{B Proofs}

This appendix provides the proofs of the main results stated in the text. We start with a straightforward proposition, which shows that, regardless of the structure of the underlying network of technological compatibilities $\mathcal{G}$, any full equilibrium network $\mathbf{G}^{*}$ consists of a collection of trees terminating at a root 
vertex in the final good industry. In the remainder of the proofs, we rely on this result and restrict our attention to such simple structures. Note that we provide the proof of Theorem 2 after that of Theorem 4 , since the former relies on a result established in the proof of the latter theorem.

\section{Proof of Theorem 1}

Proof of part (a) Since Assumption 3 implies Assumption 3', this statement follows from Theorem 10 (a).

Proof of part (c) Equation (4) follows from marginal cost pricing. More specifically, since generic producers are competitive, it must be the case that $p_{i}^{b}=c_{i}^{b}$ for all generic producers $i^{b}$. On the other hand, Assumption 3 implies that the marginal cost of generic producer $i^{b}$ is $c_{i}^{b}=w+\sum_{j=1}^{n} p_{j}^{b} / B_{i j}$. Putting the two together establishes (4).

Next, we show that customized prices in any bargaining equilibrium satisfy the system of equation (5). By Theorem 10(c), the vector of customized prices satisfies (16) and (17). Rewriting the function $f_{i j}\left(p_{i j}\right)$ in equation (17), we have

$$
f_{i j}\left(p_{i j}\right)=\delta_{i j} \mu_{i} y_{i} \frac{\partial\left(\mu_{j} y_{j}\right)}{\partial p_{i j}}+\left(1-\delta_{i j}\right) \mu_{j} y_{j} \frac{\partial\left(\mu_{i} y_{i}\right)}{\partial p_{i j}}=0,
$$

where we are using the fact that $\pi_{i}=\mu_{i} y_{i}$, in which $\mu_{i}$ and $y_{i}$ denote the markup and output of firm $i$. Since $i$ 's production function is Leontief (Assumption 3), $y_{j}=y_{i} / A_{i j}$. As a result,

$$
f_{i j}\left(p_{i j}\right)=\delta_{i j} \mu_{i} y_{i}^{2} / A_{i j}-\left(1-\delta_{i j}\right) \mu_{j} y_{i}^{2} / A_{i j}^{2}=0,
$$

where we are using the fact $\partial \mu_{j} / \partial p_{i j}=1$ and $\partial \mu_{i} / \partial p_{i j}=-1 / A_{i j}$. This implies that, as long as the outside option does not bind, $\delta_{i j} \mu_{i} A_{i j}=\left(1-\delta_{i j}\right) \mu_{j}$. However, the agreement price between $i$ and $j$ cannot exceed $i$ 's outside option of using the generic input $j^{b}$, i.e., $p_{i j}^{a} \leq p_{j}^{b} A_{i j} / B_{i j}$. Hence, pairwise customized prices in any bargaining equilibrium satisfy (5) for all $(j, i) \in \mathbf{G}$.

Proof of part (b) The existence of the bargaining equilibrium is a special case of Theorem 10(b). The statement that all pairs $(j, i) \in \mathbf{G}$ reach an agreement in any bargaining equilibrium also follows from Theorem 10(b). Furthermore, statement (c) of Theorem 10 establishes that the vector of generic prices $\boldsymbol{p}^{b}$ is unique (up to a scaling). Therefore, to establish the uniqueness of the bargaining equilibrium, it is sufficient to prove that there exists a unique vector of customized prices $\boldsymbol{p}^{a}$ that solves system of equations (5).

We start by defining a few objects. Let $\Delta$ denote a diagonal matrix indexed by the set of active customized firms in $\mathbf{G}$, with the corresponding diagonal entries given by the $\delta_{i j} .{ }^{27}$ Let $\mathbf{A}$ and $\mathbf{A}^{\dagger}$ denote square matrices with rows and columns indexed by the set of active customized firms, with the $(i, j)$ elements given by $A_{i j}$ and $1 / A_{i j}$, respectively, if $(j, i) \in \mathbf{G}$ and 0 otherwise. Finally, we define the square matrix $\mathbf{S}$ with zero diagonal as $S_{j k}=\mathbf{1}_{\{(0, j) \in \mathbf{G}\}} \mathbf{1}_{\{(0, k) \in \mathbf{G}\}}$, that is, $S_{j k}=1$ if $j$ and $k$ have a common customer in the final good industry.

\footnotetext{
${ }^{27}$ Recall that, by Assumption 1, each firm $j$ faces a single customer. As a result, there is a one-to-one correspondence between the set of customized firms in $\mathbf{G}$ and the directed edges.
} 
Having defined the above objects, now consider a pair of supplier-customer firms $(j, i) \in \mathbf{G}$. By equation (5), the agreement price, $p_{i j}^{a}$, between this pair of firms in any bargaining equilibrium satisfies

$$
\min \left\{\delta_{i j} A_{i j} \mu_{i}-\left(1-\delta_{i j}\right) \mu_{j}, \bar{p}_{i j}-p_{i j}^{a}\right\}=0
$$

where $\bar{p}_{i j}=p_{j}^{b} A_{i j} / B_{i j}$ and $\mu_{i}$ and $\mu_{j}$ are the markups of $i$ and $j$, respectively. Let $k$ denote $i$ 's customer in G. We have, $\min \left\{\delta_{i j} A_{i j}\left(p_{k i}^{a}-c_{i}\right)-\left(1-\delta_{i j}\right)\left(p_{i j}^{a}-c_{j}\right), \bar{p}_{i j}-p_{i j}^{a}\right\}=0$, in which $c_{i}$ and $c_{j}$ denote marginal costs of $i$ and $j$, respectively. Consequently,

$$
p_{i j}^{a}=\min \left\{\delta_{i j} p_{i j}^{a}+\delta_{i j} A_{i j} p_{k i}^{a}+\left(1-\delta_{i j}\right) c_{j}-\delta_{i j} c_{i} A_{i j}, \bar{p}_{i j}\right\}
$$

for all $(j, i) \in \mathbf{G}$. Hence, $\boldsymbol{p}^{a}=\left(p_{i j}^{a}\right)_{(j, i) \in \mathbf{G}}$ is a vector of customized prices in a bargaining equilibrium if and only if it satisfies

$$
\boldsymbol{p}^{a}=\min \left\{\varphi\left(\boldsymbol{p}^{a}\right), \overline{\boldsymbol{p}}\right\}
$$

where

$$
\varphi\left(\boldsymbol{p}^{a}\right)=\Delta \boldsymbol{p}^{a}+\Delta \mathbf{A}^{\prime} \boldsymbol{p}^{a}+(I-\Delta) \mathbf{A}^{\dagger} \boldsymbol{p}^{a}-\Delta\left(\mathbf{A}^{\prime} \mathbf{A}^{\dagger}+\mathbf{S}\right) \boldsymbol{p}^{a}+e,
$$

$e$ is some vector that is independent of $\boldsymbol{p}^{a}$, and we are using the fact that $c_{i}=\left(\mathbf{A}^{\dagger} \boldsymbol{p}^{a}\right)_{i}+w$. Therefore, to establish that the bargaining equilibrium is unique, it is sufficient to prove that equation (B.1) has a unique solution $\boldsymbol{p}^{a}$. We establish this claim using a series of lemmas stated and proved below.

Lemma B.1. Equation (B.1) has a unique solution $\boldsymbol{p}^{a}$ if and only if the complementarity problem

$$
q_{i j} \geq 0 \quad, \quad \tilde{\varphi}_{i j}(\boldsymbol{q}) \geq 0 \quad, \quad q_{i j} \tilde{\varphi}_{i j}(\boldsymbol{q})=0 \quad \forall(j, i) \in \mathbf{G}
$$

has a unique solution $\boldsymbol{q}$, where $\tilde{\varphi}(\boldsymbol{q})=\boldsymbol{q}-\overline{\boldsymbol{p}}+\varphi(\overline{\boldsymbol{p}}-\boldsymbol{q})$.

Proof. We prove this result by establishing a one-to-one correspondence between the solutions of (B.1) and (B.3). Note that, by (B.1), $\overline{\boldsymbol{p}}-\boldsymbol{p}^{a}=\max \left\{\overline{\boldsymbol{p}}-\varphi\left(\boldsymbol{p}^{a}\right), 0\right\}$. Let $\boldsymbol{q}=\overline{\boldsymbol{p}}-\boldsymbol{p}^{a}$. This implies that $\boldsymbol{q}=\max \{\overline{\boldsymbol{p}}-\varphi(\overline{\boldsymbol{p}}-\boldsymbol{q}), 0\}$. Hence, $\boldsymbol{p}^{a}$ is a fixed point of (B.1) if and only if

$$
\boldsymbol{q}=\max \{\boldsymbol{q}-\tilde{\varphi}(\boldsymbol{q}), 0\} .
$$

Therefore, it is sufficient to show that $\boldsymbol{q}$ satisfies (B.4) if and only if it is a solution to the complementarity problem (B.3).

First, suppose $\boldsymbol{q}$ solves (B.4). It is trivial that $\boldsymbol{q} \geq 0$. Furthermore, the fact that $\boldsymbol{q} \geq \boldsymbol{q}-\tilde{\varphi}(\boldsymbol{q})$ implies that $\tilde{\varphi}(\boldsymbol{q}) \geq 0$. Finally, note that if $q_{i j}>0$ for some $(j, i) \in \mathbf{G}$, then (B.4) implies that $q_{i j}=q_{i j}-\tilde{\varphi}_{i j}(\boldsymbol{q})$, which guarantees that $q_{i j} \tilde{\varphi}_{i j}(\boldsymbol{q})=0$. Therefore, $\boldsymbol{q}$ is a solution to (B.3).

To prove the converse implication, suppose $\boldsymbol{q}$ is a solution to (B.3). If $q_{i j}=0$ for some $(j, i) \in \mathbf{G}$, then it is immediate that $q_{i j}=\max \left\{q_{i j}-\tilde{\varphi}_{i j}(\boldsymbol{q}), 0\right\}$, where we are using the fact that any solution to (B.3) satisfies $\tilde{\varphi}_{i j}(\boldsymbol{q}) \geq 0$. On the other hand, if $q_{i j}>0$, then (B.3) implies that $\tilde{\varphi}_{i j}(\boldsymbol{q})=0$, hence once again guaranteeing that $q_{i j}=\max \left\{q_{i j}-\tilde{\varphi}_{i j}(\boldsymbol{q}), 0\right\}$. Thus, $\boldsymbol{q}$ is also a solution to (B.4).

Lemma B.1 implies that the bargaining equilibrium is unique if there is a unique $q$ that satisfies (B.3). Our next lemma provides a sufficient condition for (B.3) to have a unique solution. 
Lemma B.2. Complementarity problem (B.3) has a unique solution q if

$$
\Omega=(I-\Delta)\left(I-\mathbf{A}^{\dagger}\right)-\Delta \mathbf{A}^{\prime}+\Delta\left(\mathbf{A}^{\prime} \mathbf{A}^{\dagger}+\mathbf{S}\right),
$$

is a P-matrix. ${ }^{28}$

Proof. Recall that mapping $\varphi\left(\boldsymbol{p}^{a}\right)$ is given by (B.2). Therefore, the mapping $\tilde{\varphi}(\boldsymbol{q})=\boldsymbol{q}-\overline{\boldsymbol{p}}+\varphi(\overline{\boldsymbol{p}}-\boldsymbol{q})$ in Lemma B.1 is given by

$$
\tilde{\varphi}(\boldsymbol{q})=\Omega \boldsymbol{q}+\tilde{e}
$$

where $\Omega$ is given by (B.5) and $\tilde{e}$ is some vector that is independent of $\boldsymbol{q}$. The linearity of $\tilde{\varphi}(\boldsymbol{q})$ in $\boldsymbol{q}$ implies that (B.3) is a linear complementarity problem (Berman, 1981). Thus, by Berman (1981, p. 251), it has a unique solution if $\Omega$ is a P-matrix.

The juxtaposition of Lemmas B.1 and B.2 establishes that the economy has a unique bargaining equilibrium as long as $\Omega$ in (B.5) is a P-matrix. The following lemma establishes this claim and hence completes the proof.

Lemma B.3. Matrix $\Omega$ in (B.5) is a P-matrix.

Proof. Recall that the rows and columns of $\Omega$ are indexed by the pairs of active customized firms $(j, i) \in$ G. For the purpose of this proof, we find it more convenient to index the rows and columns of $\Omega$ by the index of the corresponding supplier firm $j$. Similarly, we use $\delta_{j}$ to denote $\delta_{i j}$. Note that we can do so unambiguously, as each customized producer $j$ has exactly one customer $i$ in G.

To prove $\Omega$ is a P-matrix, suppose to the contrary that it is not. By Berman (1981, p. 251), there exists a non-zero vector $\xi$ such that $\xi_{j}(\Omega \xi)_{j} \leq 0$ for all $j$. In particular, for any $(j, i) \in \mathbf{G}$,

$$
\xi_{j}(\Omega \xi)_{j}=\xi_{j}\left(\xi_{j}-\left(1-\delta_{j}\right) \sum_{r:(r, j) \in \mathbf{G}} \frac{\xi_{r}}{A_{j r}}-\delta_{j} A_{i j} \xi_{i}+\delta_{j} \sum_{\substack{(k, i) \in \mathbf{G} \\ k \neq j}} \frac{A_{i j}}{A_{i k}} \xi_{k}\right) \leq 0 .
$$

Given firm $j$, consider the sequence of downstream customized firms $\left(j, k_{1}, k_{2}, \ldots, k_{s}, 0\right)$ in $\mathbf{G}$ that connect $j$ to the corresponding firm in the final good producing industry and consider the following change of variables:

$$
\hat{\xi}_{j}=\xi_{j} \frac{\sqrt{1 / \delta_{j}} \sqrt{1 / \delta_{k_{1}}-1} \ldots \sqrt{1 / \delta_{k_{s}}-1}}{A_{k_{1} j} A_{k_{2} k_{1}} \ldots A_{k_{s} k_{s-1}}},
$$

where note that $k_{1}=i$ as $j$ has a single customer. Rewriting (B.6) in terms of $\hat{\xi}$ then implies that

$$
\hat{\xi}_{j}\left(\hat{\xi}_{j}-\sum_{r:(r, j) \in \mathbf{G}} \hat{\xi}_{r} \sqrt{\delta_{r}\left(1-\delta_{j}\right)}-\hat{\xi}_{i} \sqrt{\delta_{j}\left(1-\delta_{i}\right)}+\sum_{\substack{(k, i) \in \mathbf{G} \\ k \neq j}} \hat{\xi}_{k} \sqrt{\delta_{j} \delta_{k}}\right) \leq 0
$$

\footnotetext{
${ }^{28} \mathrm{~A}$ square matrix is a $P$-matrix if all its principal minors are strictly positive.
} 
for all $j$. Summing both sides of the above inequality therefore leads to

$$
\sum_{j:(j, i) \in \mathbf{G}} \hat{\xi}_{j}^{2}-2 \sum_{j:(j, i) \in \mathbf{G}} \hat{\xi}_{j} \hat{\xi}_{i} \sqrt{\delta_{j}\left(1-\delta_{i}\right)}+\sum_{j:(j, i) \in \mathbf{G}} \sum_{\substack{(k, i) \in \mathbf{G} \\ k \neq j}} \hat{\xi}_{k} \hat{\xi}_{j} \sqrt{\delta_{j} \delta_{k}} \leq 0
$$

which in turn implies that

$$
\sum_{i:(i, 0) \in \mathbf{G}} \delta_{i} \hat{\xi}_{i}^{2}+\sum_{j:(j, i) \in \mathbf{G}}\left(\hat{\xi}_{j} \sqrt{1-\delta_{j}}-\sum_{r:(r, j) \in \mathbf{G}} \hat{\xi}_{r} \sqrt{\delta_{r}}\right)^{2} \leq 0,
$$

where the first sum is over all firms $i$ that sell to the firm in the final good producing industry 0 . It is immediate that both terms on the left-hand side of the above inequality are non-negative, which implies that they both must be equal to zero. As a result, $\hat{\xi}_{i}=0$ for all firms $i$ that sell to the firm in the final good producing industry and

$$
\hat{\xi}_{j} \sqrt{1-\delta_{j}}=\sum_{r:(r, j) \in \mathbf{G}^{*}} \hat{\xi}_{r} \sqrt{\delta_{r}}
$$

for all firms $j$. In particular, (B.7) implies that $\hat{\xi}_{j}=0$ for all firms $j$ with no customized suppliers in G. Now using (B.7) iteratively over the production network $\mathbf{G}$ implies that the only solution to the above system of equations is that $\hat{\xi}_{j}=0$ for all $j \in \mathbf{G}$, thus contradicting the assumption that $\xi$ is non-zero. This establishes that $\Omega$ is a P-matrix.

\section{Proof of Theorem 3}

We start with a preliminary lemma, which establishes that all firm-level prices (and hence markups) are continuous in bargaining powers and productivity shocks.

Lemma B.4. For any given production network of active firms $\mathbf{G}$, the bargaining equilibrium $\left(\boldsymbol{p}^{a}, \boldsymbol{p}^{b}\right)$ is continuous in bargaining powers and productivity shocks.

Proof. Let $\delta=\left(\delta_{i j}\right)_{(j, i) \in \mathbf{G}}$ denote the vector of bargaining powers in the production network $\mathbf{G}$ and define the correspondence $\Phi$ that maps the vector of bargaining powers $\delta$ to the set of all bargaining equilibria consistent with $\delta$, i.e.,

$$
\Phi(\delta)=\left\{\left(\boldsymbol{p}^{a}, \boldsymbol{p}^{b}\right):\left(\boldsymbol{p}^{a}, \boldsymbol{p}^{b}\right) \text { is a bargaining equilibrium given } \delta\right\} .
$$

Since the graph of this correspondence is closed, $\Phi$ is upper hemi-continuous. Furthermore, recall from Theorem 1(b) that the bargaining equilibrium is always unique, implying that $\Phi$ is a single-valued function. This coupled with upper hemi-continuity of $\Phi$ guarantees that the mapping from the vector of bargaining powers to the bargaining equilibrium is continuous.

The proof of the continuity of the bargaining equilibrium in productivities is analogous.

With the above lemma in hand, we are now ready to present the proof of Theorem 3. For brevity, we only present the proof for firms that are upstream to $j$. The result for downstream and horizontal firms follows from a similar argument. 
Proof of part (a) Let $\delta=\left(\delta_{i j}\right)_{(j, i) \in \mathbf{G}}$ denote the vector of bargaining powers in the production network G. Recall from equation (B.1) that, for any $\delta$, the vector of equilibrium customized prices $\boldsymbol{p}^{a}$ is the unique solution to the system of equations $\boldsymbol{p}^{a}=\min \left\{\varphi\left(\boldsymbol{p}^{a}, \delta\right), \overline{\boldsymbol{p}}\right\}$. For any given $\delta$, define the partition $\mathcal{S}(\delta)$ of the set of active firms in $\mathbf{G}$ such that the parties in a supplier-customer pair $(j, i) \in \mathbf{G}$ belong to the same partition element if and only if the outside option in their pairwise bargaining game is strictly loose in equilibrium, that is, $p_{i j}^{a}=\varphi_{i j}(\boldsymbol{p}, \delta)<\bar{p}_{i j}$. Thus, the two firms belong to two different partition elements if $p_{i j}^{a}=\bar{p}_{i j}$.

Fix a supplier-customer pair of firms $(j, i) \in \mathbf{G}$ and all bargaining powers $\delta_{-i j}$ other than $\delta_{i j}$. Define the sequence $0=\delta_{i j}^{(1)}<\delta_{i j}^{(2)}<\cdots<\delta_{i j}^{(m)}=1$ and the resulting collection of non-overlapping intervals $I_{k}=\left(\delta_{i j}^{(k)}, \delta_{i j}^{(k+1)}\right)$ such that

$$
\begin{array}{ll}
\mathcal{S}\left(\delta_{i j}, \delta_{-i j}\right)=\mathcal{S}\left(\delta_{i j}^{\prime}, \delta_{-i j}\right) & \text { if } \delta_{i j}, \delta_{i j}^{\prime} \in I_{k} \\
\mathcal{S}\left(\delta_{i j}, \delta_{-i j}\right) \neq \mathcal{S}\left(\delta_{i j}^{\prime}, \delta_{-i j}\right) & \text { if } \delta_{i j} \in I_{k}, \delta_{i j}^{\prime} \in I_{k}^{\prime} \text { and } k \neq k^{\prime} .
\end{array}
$$

Thus, the partition $\mathcal{S}\left(\delta_{i j}, \delta_{-i j}\right)$-and hence, the collection of pairwise bargaining problems in $\mathbf{G}$ for which the outside option constraint binds-remains unchanged for all $\delta_{i j}$ within the same interval $I_{k}$. Since there are only finitely many such intervals, if all markups upstream to $j$ are weakly increasing in $\delta_{i j}$ in the interval $I_{k}$ for all $1 \leq k<m$, then Lemma B.4 guarantees that they are weakly increasing in $\delta_{i j}$ over the entire $[0,1]$ interval.

To establish that all markups upstream to $j$ are weakly increasing within interval $I_{k}$ for all $1 \leq k<$ $m$, fix an interval $I_{k}=\left(\delta_{i j}^{(k)}, \delta_{i j}^{(k+1)}\right)$ and let $\mathcal{S}^{(k)}=\mathcal{S}\left(\delta_{i j}, \delta_{-i j}\right)$ denote the corresponding partition of $\mathbf{G}$ for all $\delta_{i j} \in I_{k}$. We consider two separate cases. First, suppose $i$ and $j$ belong to two separate partition elements. By definition, $p_{i j}^{a}=\bar{p}_{i j}$. Therefore, it is immediate that changes in $\delta_{i j}$ within the interval $I_{k}$ has no impact on any of the prices and markups in the economy, thus establishing the desired result that all markups are weakly increasing in that interval. As the second case, suppose that $i$ and $j$ belong to the same element $S$ within the partition $\mathcal{S}^{(k)}$. Once again, all prices outside of $S$ remain unchanged. On the other hand, for any pair of firms $(l, r) \in \mathbf{G}$ such that $r, l \in S$-including the pair $(j, i)$-equation (6) implies that

$$
\delta_{r l} A_{r l} \mu_{r}=\left(1-\delta_{r l}\right) \mu_{l},
$$

i.e., the markup charged by each firm is proportional to that charged by its customer. Therefore, an increase in $\delta_{i j}$ strictly increases the markup charged by $j$ and all its upstream suppliers within the partition element $S$. Taken together, the two cases establish that all markups upstream to $j$ are increasing in $\delta_{i j}$ for all $\delta_{i j} \in I_{k}$, which in turn guarantees that all upstream markups are weakly increasing in $\delta_{i j}$ over the entire interval $[0,1]$.

Proof of part (b) As a first observation, note that, when all production technologies are Leontief, all quantities are invariant to prices and hence bargaining powers. Therefore, monotonicity of markups established in part (a) of the theorem guarantees that the profits of all firms upstream to $j$ also increase in $\delta_{i j}$ over the entire interval $[0,1]$. 
Proof of part (c) By continuity of bargaining equilibrium established in Lemma B.4, it is sufficient to show that all upstream prices are increasing in $\delta_{i j}$ within a given interval $I_{k}=\left(\delta_{i j}^{(k)}, \delta_{i j}^{(k+1)}\right) \cdot{ }^{29}$ Let $\mathcal{S}^{(k)}=\mathcal{S}\left(\delta_{i j}, \delta_{-i j}\right)$ denote the corresponding partition of the set of active firms G for any $\delta_{i j} \in I_{k}$. We consider two separate cases. First, if $i$ and $j$ belong to two distinct partition elements, then all prices are invariant to the value of $\delta_{i j} \in I_{k}$, thus establishing the desired claim. Second, suppose $i$ and $j$ belong to the same partition element $S \in \mathcal{S}^{(k)}$. Once again, all prices upstream to $j$ that are outside of $S$ remain invariant to the value of $\delta_{i j} \in I_{k}$. Therefore, the proof is complete once we show that the price of all firms in $S$ that are also upstream to $j$ are increasing in $\delta_{i j}$ for all $\delta_{i j} \in I_{k}$. We prove this claim by induction. Consider the set of firms $S_{0} \subseteq S$ that are upstream to $j$ and have no customized supplier in $S$. The marginal cost of any firm in $S_{0}$ is invariant to $\delta_{i j}$, while at the same time, its markup is increasing in $\delta_{i j}$ as established in part (a). Therefore, it must be the case that its output price is increasing in $\delta_{i j}$. Next define the set of upstream firms $S_{1} \subseteq S$ such that they have at least one customized supplier in $S_{0}$ and no customized suppliers in $S \backslash S_{0}$. The fact that the output price of firms in $S_{0}$ are increasing in $\delta_{i j}$ implies that the marginal cost of firms in $S_{1}$ are also increasing in $\delta_{i j}$. But recall that the markup of any firm that are upstream to $j$ (including those in $S_{1}$ ) are increasing in $\delta_{i j}$. Therefore, it must be the case that the output price of firms in $S_{1}$ are increasing in $\delta_{i j}$. Iterating this argument over set $S$ establishes the desired claim and completes the proof.

\section{Proof of Theorem 4}

Proof of part (a) Consider two feasible production networks $\underline{\mathbf{G}}, \overline{\mathbf{G}} \subseteq \mathcal{G}$ such that $\underline{\mathbf{G}} \subseteq \overline{\mathbf{G}}$. Denote the vectors of customized prices in the two economies as functions of pairwise bargaining powers by $\boldsymbol{p}^{a}(\underline{\mathbf{G}}, \underline{\delta})$ and $\boldsymbol{p}^{a}(\overline{\mathbf{G}}, \bar{\delta})$, respectively, where $\underline{\delta}=\left(\delta_{i j}\right)_{(j, i) \in \underline{\mathbf{G}}}$ and $\bar{\delta}=\left(\delta_{i j}\right)_{(j, i) \in \overline{\mathbf{G}}}$ are the corresponding vectors of bargaining powers. Since $\underline{\mathbf{G}} \subseteq \overline{\mathbf{G}}$, we can define the boundary of $\underline{\mathbf{G}}$ as

$$
\partial \underline{\mathbf{G}}=\{(j, i) \in \overline{\mathbf{G}}: i \in \underline{\mathbf{G}} \text { and } j \notin \underline{\mathbf{G}}\}
$$

that is, the set of supplier-customer pairs in $\overline{\mathbf{G}}$ such that the customer belongs to $\mathbf{G}$ but the supplier does not. Accordingly, we can express the vector of bargaining powers in $\overline{\mathbf{G}}$ as $\bar{\delta}=\left(\underline{\delta}, \delta^{\partial}, \delta^{e}\right)$, where $\delta^{\partial}=\left(\delta_{i j}\right)_{(j, i) \in \partial \underline{\mathbf{G}}}$ is the vector of bargaining powers between pairs of firms on the boundary of $\underline{\mathbf{G}}$ and $\delta^{e}$ is the vector of bargaining powers between pairs of firms where both parties belong to $\overline{\mathbf{G}}$ but not $\underline{\mathbf{G}}$.

The key observation is that

$$
p_{i j}^{a}(\underline{\mathbf{G}}, \underline{\delta})=p_{i j}^{a}\left(\overline{\mathbf{G}},\left(\underline{\delta}, 1, \delta^{e}\right)\right) \quad \text { for all }(j, i) \in \underline{\mathbf{G}},
$$

that is, pairwise equilibrium prices in $\mathbf{G}$ coincide with pairwise prices in $\overline{\mathbf{G}}$ if all bargaining powers between the pairs of firms on the boundary of $\underline{G}$ are set to 1 . This is a consequence of the fact that, in any pairwise bargaining, the customer firm is indifferent between the customized and generic variant of a good if the corresponding customized supplier has all the bargaining power. Equation (B.8) thus implies that

$$
\mu_{i}(\underline{\mathbf{G}}, \underline{\delta})=\mu_{i}\left(\overline{\mathbf{G}},\left(\underline{\delta}, 1, \delta^{e}\right)\right) \quad \text { for all } i \in \underline{\mathbf{G}} .
$$

\footnotetext{
${ }^{29}$ See the proof of part (a) of the theorem for the definition of these intervals.
} 
The above equality, in juxtaposition with the monotonicity of markups with respect to the bargaining powers established in Theorem 3(a), then leads to

$$
\mu_{i}(\underline{\mathbf{G}}, \underline{\delta})=\mu_{i}\left(\overline{\mathbf{G}},\left(\underline{\delta}, 1, \delta^{e}\right)\right) \leq \mu_{i}(\overline{\mathbf{G}}, \bar{\delta}) \quad \text { for all } i \in \underline{\mathbf{G}},
$$

where we are using the fact that all firms in $\underline{\mathbf{G}}$ are either downstream or horizontal to $\partial \underline{\mathbf{G}}$.

Proof of part (b) The representative household's budget constraints under the two production networks are given by

$$
\begin{aligned}
& y_{0}(\overline{\mathbf{G}})=w L+\sum_{j \in \overline{\mathbf{G}}}\left(\pi_{j}(\overline{\mathbf{G}})-w z_{j}\right) \\
& y_{0}(\underline{\mathbf{G}})=w L+\sum_{j \in \underline{\mathbf{G}}}\left(\pi_{j}(\underline{\mathbf{G}})-w z_{j}\right),
\end{aligned}
$$

where $y_{0}$ denotes the household's demand for the final good and we are choosing the final good as the numeraire. Therefore,

$$
y_{0}(\overline{\mathbf{G}}) \geq y_{0}(\underline{\mathbf{G}})+\sum_{j \in \underline{\mathbf{G}}}\left(\pi_{j}(\overline{\mathbf{G}})-\pi_{j}(\underline{\mathbf{G}})\right),
$$

where the inequality follows from condition (8). Since all production functions are Leontief, firm-level outputs are proportional to the household's demand for the final good, i.e., $y_{j}=\alpha_{j} y_{0}$, where $\alpha_{j}$ is a constant that is independent of prices and quantities (though it may depend on the productivities). Consequently,

$$
y_{0}(\overline{\mathbf{G}}) \geq y_{0}(\underline{\mathbf{G}})+\sum_{j \in \underline{\mathbf{G}}}\left(\alpha_{j} \mu_{j}(\overline{\mathbf{G}}) y_{0}(\overline{\mathbf{G}})-\alpha_{j} \mu_{j}(\underline{\mathbf{G}}) y_{0}(\underline{\mathbf{G}})\right) \text {. }
$$

By part (a) of Theorem $4, \mu_{j}(\overline{\mathbf{G}}) \geq \mu_{j}(\underline{\mathbf{G}})$ for all $j \in \underline{\mathbf{G}}$. Therefore,

$$
\left(y_{0}(\overline{\mathbf{G}})-y_{0}(\underline{\mathbf{G}})\right)\left(1-\sum_{j \in \underline{\mathbf{G}}} \alpha_{j} \mu_{j}(\overline{\mathbf{G}})\right) \geq 0 .
$$

Since $\overline{\mathbf{G}}$ is feasible by assumption, (B.9) guarantees that

$$
y_{0}(\overline{\mathbf{G}})>\sum_{j \in \overline{\mathbf{G}}} \pi_{j}(\overline{\mathbf{G}})=y_{0}(\overline{\mathbf{G}}) \sum_{j \in \overline{\mathbf{G}}} \alpha_{j} \mu_{j}(\overline{\mathbf{G}}) \geq y_{0}(\overline{\mathbf{G}}) \sum_{j \in \underline{\mathbf{G}}} \alpha_{j} \mu_{j}(\overline{\mathbf{G}}) .
$$

The juxtaposition of the last two inequalities therefore implies that $y_{0}(\overline{\mathbf{G}}) \geq y_{0}(\underline{\mathbf{G}})$.

Proof of part (c) Since all production functions are Leontief, the gross profit of firm $i$ is given by $\pi_{i}=$ $\alpha_{i} \mu_{i} y_{0}$, where $\alpha_{i}$ is a constant that is independent of prices and quantities. In part (a), we established that $\mu_{i}(\underline{\mathbf{G}}) \leq \mu_{i}(\overline{\mathbf{G}})$ for all $i \in \underline{\mathbf{G}}$, whereas in part (b), we established that $y_{0}(\underline{\mathbf{G}}) \leq y_{0}(\overline{\mathbf{G}})$. Therefore, it is immediate that $\pi_{i}(\underline{\mathbf{G}}) \leq \pi_{i}(\overline{\mathbf{G}})$ for all $i \in \underline{\mathbf{G}}$. 


\section{Proof of Theorem 2}

Proof of parts (a) and (b) By Definition $3, \mathbf{G}^{*} \subseteq \mathcal{G}$ corresponds to a full equilibrium if and only if it is the fixed point of the mapping $\phi: 2^{\mathcal{G}} \rightarrow 2^{\mathcal{G}}$, defined as

$$
\phi(\mathbf{G})=\left\{i \in \mathcal{G}: \pi_{i}(\{i\} \cup \mathbf{G}) \geq w z_{i}\right\} .
$$

If this mapping satisfies Assumptions A.1 and A.2, then Theorem A.1 guarantees that $\phi$ has a greatest fixed point, thus establishing parts (a) and (b) of Theorem 2. Therefore, we first verify that $\phi$ satisfies Assumptions A.1 and A.2 and then prove part (c) of Theorem 2.

Assumption A.1: To establish that $\phi$ satisfies Assumption A.1, take two production networks of active firms $\mathbf{G}_{1}, \mathbf{G}_{2} \subseteq \mathcal{G}$ and suppose that $\mathbf{G}_{1} \subseteq \phi\left(\mathbf{G}_{2}\right)$. Hence,

$$
\pi_{i}\left(\{i\} \cup \mathbf{G}_{2}\right) \geq w z_{i} \quad \text { for all } i \in \mathbf{G}_{1} .
$$

As in the proof of Theorem 4(b), the fact that all production technologies are Leontief implies that the above inequality can be rewritten as

$$
\alpha_{i} \mu_{i}\left(\mathbf{G}_{2} \cup\{i\}\right) y_{0}\left(\mathbf{G}_{2} \cup\{i\}\right) \geq w z_{i} \quad \text { for all } i \in \mathbf{G}_{1},
$$

where $\mu_{i}$ is the markup of firm $i$ and $\alpha_{i}$ is a constant independent of prices and quantities that relates $i$ 's output to household's demand for consumption good via $y_{i}=\alpha_{i} y_{0}$. Furthermore, when the set of active firms is $\mathbf{G}_{2} \cup\{i\}$, the household's budget constraint implies that

$$
y_{0}\left(\mathbf{G}_{2} \cup\{i\}\right)=w L+y_{0}\left(\mathbf{G}_{2} \cup\{i\}\right) \sum_{j \in \mathbf{G}_{2} \cup\{i\}} \alpha_{j} \mu_{j}\left(\mathbf{G}_{2} \cup\{i\}\right)-\sum_{j \in \mathbf{G}_{2} \cup\{i\}} w z_{j},
$$

where the first term on the right-hand side above is the household's labor income and the other two terms are the customized firms' profits net of their fixed costs. Solving for $y_{0}\left(\mathbf{G}_{2} \cup\{i\}\right)$ from the above equation and plugging it into (B.11) implies that whenever $\mathbf{G}_{1} \subseteq \phi\left(\mathbf{G}_{2}\right)$, then ${ }^{30}$

$$
z_{i} \leq \frac{\alpha_{i} \mu_{i}\left(\mathbf{G}_{2} \cup\{i\}\right)}{1-\sum_{j \in \mathbf{G}_{2}} \alpha_{j} \mu_{j}\left(\mathbf{G}_{2} \cup\{i\}\right)}\left(L-\sum_{j \in \mathbf{G}_{2}} z_{j}\right) \quad \text { for all } i \in \mathbf{G}_{1} .
$$

Furthermore, Theorem 4(a) guarantees that $\mu_{j}\left(\mathbf{G}_{1} \cup \mathbf{G}_{2}\right) \geq \mu_{j}\left(\mathbf{G}_{2} \cup\{i\}\right)$ for any pair $i$ and $j$ such that $j \in \mathbf{G}_{2} \cup\{i\}$ and all $i \in \mathbf{G}_{1}$. Consequently,

$$
z_{i} \leq \frac{\alpha_{i} \mu_{i}\left(\mathbf{G}_{1} \cup \mathbf{G}_{2}\right)}{1-\sum_{j \in \mathbf{G}_{2}} \alpha_{j} \mu_{j}\left(\mathbf{G}_{1} \cup \mathbf{G}_{2}\right)}\left(L-\sum_{j \in \mathbf{G}_{2}} z_{j}\right) \quad \text { for all } i \in \mathbf{G}_{1} .
$$

Next, consider the economy in which the set of active firms is instead $\mathbf{G}_{1} \cup \mathbf{G}_{2}$. In this case, firm $i$ 's gross profit is given by $\pi_{i}\left(\mathbf{G}_{1} \cup \mathbf{G}_{2}\right)=\alpha_{i} \mu_{i}\left(\mathbf{G}_{1} \cup \mathbf{G}_{2}\right) y_{0}\left(\mathbf{G}_{1} \cup \mathbf{G}_{2}\right)$. Therefore, solving for $y_{0}$ from the household's budget constraint and plugging it back into the expression for $\pi_{i}\left(\mathbf{G}_{1} \cup \mathbf{G}_{2}\right)$ leads to

$$
\pi_{i}\left(\mathbf{G}_{1} \cup \mathbf{G}_{2}\right)-w z_{i}=\frac{w \alpha_{i} \mu_{i}\left(\mathbf{G}_{1} \cup \mathbf{G}_{2}\right)}{1-\sum_{j \in \mathbf{G}_{1} \cup \mathbf{G}_{2}} \alpha_{j} \mu_{j}\left(\mathbf{G}_{1} \cup \mathbf{G}_{2}\right)}\left(L-\sum_{j \in \mathbf{G}_{1} \cup \mathbf{G}_{2}} z_{j}\right)-w z_{i}
$$

\footnotetext{
${ }^{30}$ Note that (B.12) holds for all $i \in \mathbf{G}_{1}$ irrespective of whether $i \in \mathbf{G}_{2}$ or not.
} 
for all $i \in \mathbf{G}_{1}$. Consequently, the upper bound on $z_{i}$ in (B.13) implies that

$$
\pi_{i}\left(\mathbf{G}_{1} \cup \mathbf{G}_{2}\right)-w z_{i} \geq w \alpha_{i} \mu_{i}\left(\mathbf{G}_{1} \cup \mathbf{G}_{2}\right)\left(\frac{L-\sum_{j \in \mathbf{G}_{1} \cup \mathbf{G}_{2}} z_{j}}{1-\sum_{j \in \mathbf{G}_{1} \cup \mathbf{G}_{2}} \alpha_{j} \mu_{j}\left(\mathbf{G}_{1} \cup \mathbf{G}_{2}\right)}-\frac{L-\sum_{j \in \mathbf{G}_{2}} z_{j}}{1-\sum_{j \in \mathbf{G}_{2}} \alpha_{j} \mu_{j}\left(\mathbf{G}_{1} \cup \mathbf{G}_{2}\right)}\right) .
$$

Simplifying the right-hand side of the above inequality, we obtain,

$$
\pi_{i}\left(\mathbf{G}_{1} \cup \mathbf{G}_{2}\right)-w z_{i} \geq \frac{w \alpha_{i} \mu_{i}\left(\mathbf{G}_{1} \cup \mathbf{G}_{2}\right)}{1-\sum_{j \in \mathbf{G}_{1} \cup \mathbf{G}_{2}} \alpha_{j} \mu_{j}\left(\mathbf{G}_{1} \cup \mathbf{G}_{2}\right)} Q
$$

for all $i \in \mathbf{G}_{1}$, where

$$
Q=\frac{\sum_{j \in \mathbf{G}_{1} \backslash \mathbf{G}_{2}} \alpha_{j} \mu_{j}\left(\mathbf{G}_{1} \cup \mathbf{G}_{2}\right)}{1-\sum_{j \in \mathbf{G}_{2}} \alpha_{j} \mu_{j}\left(\mathbf{G}_{1} \cup \mathbf{G}_{2}\right)}\left(L-\sum_{j \in \mathbf{G}_{2}} z_{j}\right)-\sum_{j \in \mathbf{G}_{1} \backslash \mathbf{G}_{2}} z_{j}
$$

On the other hand, summing both sides of inequality (B.13) over all $i \in \mathbf{G}_{1} \backslash \mathbf{G}_{2}$ guarantees that $Q \geq 0$. Therefore, it is immediate that $\pi_{i}\left(\mathbf{G}_{1} \cup \mathbf{G}_{2}\right) \geq w z_{i}$ for all $i \in \mathbf{G}_{1}$, and hence, $\mathbf{G}_{1} \subseteq \phi\left(\mathbf{G}_{1} \cup \mathbf{G}_{2}\right)$. This establishes that mapping $\phi$ in (B.10) satisfies Assumption A.1.

Assumption A.2: We now establish that mapping $\phi$ in (B.10) also satisfies Assumption A.2. Let $\mathbf{G}_{1}, \mathbf{G}_{2} \subseteq \mathcal{G}$ denote a pair of feasible production networks such that $\mathbf{G}_{k} \subseteq \phi\left(\mathbf{G}_{k}\right)$ for $k \in\{1,2\}$. The assumption that $\mathbf{G}_{k} \subseteq \phi\left(\mathbf{G}_{k}\right)$ implies that $\pi_{i}\left(\mathbf{G}_{k}\right)=\alpha_{i} \mu_{i}\left(\mathbf{G}_{k}\right) y_{0}\left(\mathbf{G}_{k}\right) \geq w z_{i}$ for all $i \in \mathbf{G}_{k}$. Solving for $y_{0}\left(\mathbf{G}_{k}\right)$ from the household's budget constraint, $y_{0}\left(\mathbf{G}_{k}\right)=w L+\sum_{j \in \mathbf{G}_{k}}\left(\alpha_{j} \mu_{j}\left(\mathbf{G}_{k}\right) y_{0}\left(\mathbf{G}_{k}\right)-w z_{j}\right)$, therefore implies that

$$
z_{i} \leq \frac{\alpha_{i} \mu_{i}\left(\mathbf{G}_{k}\right)}{1-\sum_{j \in \mathbf{G}_{k}} \alpha_{j} \mu_{j}\left(\mathbf{G}_{k}\right)}\left(L-\sum_{j \in \mathbf{G}_{k}} z_{j}\right)
$$

for all $i \in \mathbf{G}_{k}$. Furthermore, recall from Theorem 4 (a) that $\mu_{i}\left(\mathbf{G}_{k}\right) \leq \mu_{i}\left(\mathbf{G}_{1} \cup \mathbf{G}_{2}\right)$ for all $i \in \mathbf{G}_{k}$. Hence,

$$
z_{i} \leq \frac{\alpha_{i} \mu_{i}\left(\mathbf{G}_{1} \cup \mathbf{G}_{2}\right)}{1-\sum_{j \in \mathbf{G}_{k}} \alpha_{j} \mu_{j}\left(\mathbf{G}_{1} \cup \mathbf{G}_{2}\right)}\left(L-\sum_{j \in \mathbf{G}_{k}} z_{j}\right)
$$

for all $i \in \mathbf{G}_{k}$. Setting $k=1$ and summing over all $i \in \mathbf{G}_{1} \backslash \mathbf{G}_{2}$ implies that

$$
\left(\sum_{j \in \mathbf{G}_{1} \backslash \mathbf{G}_{2}} z_{j}\right)\left(1-\sum_{j \in \mathbf{G}_{1} \cap \mathbf{G}_{2}} \alpha_{j} \mu_{j}\left(\mathbf{G}_{1} \cup \mathbf{G}_{2}\right)\right) \leq\left(L-\sum_{j \in \mathbf{G}_{1} \cap \mathbf{G}_{2}} z_{j}\right)\left(\sum_{j \in \mathbf{G}_{1} \backslash \mathbf{G}_{2}} \alpha_{j} \mu_{j}\left(\mathbf{G}_{1} \cup \mathbf{G}_{2}\right)\right) .
$$

Similarly, setting $k=2$ in (B.14) and summing over all $i \in \mathbf{G}_{2} \backslash \mathbf{G}_{1}$ implies that

$$
\left(\sum_{j \in \mathbf{G}_{2} \backslash \mathbf{G}_{1}} z_{j}\right)\left(1-\sum_{j \in \mathbf{G}_{1} \cap \mathbf{G}_{2}} \alpha_{j} \mu_{j}\left(\mathbf{G}_{1} \cup \mathbf{G}_{2}\right)\right) \leq\left(L-\sum_{j \in \mathbf{G}_{1} \cap \mathbf{G}_{2}} z_{j}\right)\left(\sum_{j \in \mathbf{G}_{2} \backslash \mathbf{G}_{1}} \alpha_{j} \mu_{j}\left(\mathbf{G}_{1} \cup \mathbf{G}_{2}\right)\right) .
$$

Therefore, as long as $\mathbf{G}_{1} \subseteq \phi\left(\mathbf{G}_{1}\right)$ and $\mathbf{G}_{2} \subseteq \phi\left(\mathbf{G}_{2}\right)$, inequalities (B.15) and (B.16) are satisfied. 
Next, consider the profit $\pi_{i}\left(\mathbf{G}_{1} \cup \mathbf{G}_{2}\right)$ of an arbitrary firm $i \in \mathbf{G}_{1}$ when the production network of active firms is $\mathbf{G}_{1} \cup \mathbf{G}_{2}$. Once again, solving for household demand from her budget constraint and plugging it back to firm $i$ 's profit function implies that

$$
\begin{aligned}
\pi_{i}\left(\mathbf{G}_{1} \cup \mathbf{G}_{2}\right)-w z_{i} & =\frac{w \alpha_{i} \mu_{i}\left(\mathbf{G}_{1} \cup \mathbf{G}_{2}\right)}{1-\sum_{j \in \mathbf{G}_{1} \cup \mathbf{G}_{2}} \alpha_{j} \mu_{j}\left(\mathbf{G}_{1} \cup \mathbf{G}_{2}\right)}\left(L-\sum_{j \in \mathbf{G}_{1} \cup \mathbf{G}_{2}} z_{j}\right)-w z_{i} \\
& \geq w \alpha_{i} \mu_{i}\left(\mathbf{G}_{1} \cup \mathbf{G}_{2}\right)\left(\frac{L-\sum_{j \in \mathbf{G}_{1} \cup \mathbf{G}_{2}} z_{j}}{1-\sum_{j \in \mathbf{G}_{1} \cup \mathbf{G}_{2}} \alpha_{j} \mu_{j}\left(\mathbf{G}_{1} \cup \mathbf{G}_{2}\right)}-\frac{L-\sum_{j \in \mathbf{G}_{1}} z_{j}}{1-\sum_{j \in \mathbf{G}_{1}} \alpha_{j} \mu_{j}\left(\mathbf{G}_{1} \cup \mathbf{G}_{2}\right)}\right)
\end{aligned}
$$

for all $i \in \mathbf{G}_{1}$, where the inequality is a consequence of (B.14). Simplifying the right-hand side of the above inequality therefore implies that $\pi_{i}\left(\mathbf{G}_{1} \cup \mathbf{G}_{2}\right)-w z_{i} \geq 0$ for all $i \in \mathbf{G}_{1}$ as long as $Q^{\prime}>0$, where

$$
Q^{\prime}=\left(L-\sum_{j \in \mathbf{G}_{1}} z_{j}\right)\left(\sum_{j \in \mathbf{G}_{2} \backslash \mathbf{G}_{1}} \alpha_{j} \mu_{j}\left(\mathbf{G}_{1} \cup \mathbf{G}_{2}\right)\right)-\left(1-\sum_{j \in \mathbf{G}_{1}} \alpha_{j} \mu_{j}\left(\mathbf{G}_{1} \cup \mathbf{G}_{2}\right)\right)\left(\sum_{j \in \mathbf{G}_{2} \backslash \mathbf{G}_{1}} z_{j}\right) .
$$

By inequality (B.16), we have

$$
Q^{\prime} \geq\left(\sum_{j \in \mathbf{G}_{2} \backslash \mathbf{G}_{1}} z_{j}\right)\left(\frac{L-\sum_{j \in \mathbf{G}_{1}} z_{j}}{L-\sum_{j \in \mathbf{G}_{1} \cap \mathbf{G}_{2}} z_{j}}\left(1-\sum_{j \in \mathbf{G}_{1} \cap \mathbf{G}_{2}} \alpha_{j} \mu_{j}\left(\mathbf{G}_{1} \cup \mathbf{G}_{2}\right)\right)-\left(1-\sum_{j \in \mathbf{G}_{1}} \alpha_{j} \mu_{j}\left(\mathbf{G}_{1} \cup \mathbf{G}_{2}\right)\right)\right),
$$

and as a result

$$
\begin{aligned}
Q^{\prime} \geq\left(\frac{\sum_{j \in \mathbf{G}_{2} \backslash \mathbf{G}_{1}} z_{j}}{L-\sum_{j \in \mathbf{G}_{1} \cap \mathbf{G}_{2}} z_{j}}\right) & {\left[\left(L-\sum_{j \in \mathbf{G}_{1} \cap \mathbf{G}_{2}} z_{j}\right)\left(\sum_{j \in \mathbf{G}_{1} \backslash \mathbf{G}_{2}} \alpha_{j} \mu_{j}\left(\mathbf{G}_{1} \cup \mathbf{G}_{2}\right)\right)\right.} \\
& \left.-\left(1-\sum_{j \in \mathbf{G}_{1} \cap \mathbf{G}_{2}} \alpha_{j} \mu_{j}\left(\mathbf{G}_{1} \cup \mathbf{G}_{2}\right)\right)\left(\sum_{j \in \mathbf{G}_{1} \backslash \mathbf{G}_{2}} z_{j}\right)\right] .
\end{aligned}
$$

Now (B.15) guarantees that the right-hand side of the above inequality is non-negative. Therefore, we just established that $\pi_{i}\left(\mathbf{G}_{1} \cup \mathbf{G}_{2}\right)-w z_{i} \geq 0$ for all $i \in \mathbf{G}_{1}$. An identical argument establishes that $\pi_{i}\left(\mathbf{G}_{1} \cup \mathbf{G}_{2}\right)-w z_{i} \geq 0$ for all $i \in \mathbf{G}_{2}$.

Taken together, we have, $\pi_{i}\left(\mathbf{G}_{1} \cup \mathbf{G}_{2}\right)-w z_{i} \geq 0$ for all $i \in \mathbf{G}_{1} \cup \mathbf{G}_{2}$. Therefore $\mathbf{G}_{1} \cup \mathbf{G}_{2} \subseteq \phi\left(\mathbf{G}_{1} \cup \mathbf{G}_{2}\right)$ for any arbitrary pair of feasible production networks $\mathbf{G}_{1}$ and $\mathbf{G}_{2}$ such that $\mathbf{G}_{1} \subseteq \phi\left(\mathbf{G}_{1}\right)$ and $\mathbf{G}_{2} \subseteq$ $\phi\left(\mathbf{G}_{2}\right)$, thus implying that Assumption A.2 is satisfied.

Having proven mapping $\phi$ in (B.10) satisfies Assumptions A.1 and A.2, Theorem A.1 establishes parts (a) and (b) of Theorem 2.

Proof of part (c) Let $\mathbf{G}^{*}$ denote the production network of active firms corresponding to an arbitrary full equilibrium. The household's budget constraint under $\mathrm{G}^{*}$ is given by

$$
y_{0}\left(\mathbf{G}^{*}\right)=w L+\sum_{i \in \mathbf{G}^{*}}\left(\pi_{i}\left(\mathbf{G}^{*}\right)-w z_{i}\right)
$$


Similarly, if $\mathbf{G}_{\max }^{*}$ denotes the greatest full equilibrium (so that $\mathbf{G}^{*} \subseteq \mathbf{G}_{\max }^{*}$ for any full equilibrium $\mathbf{G}^{*}$ ), we have

$$
\begin{aligned}
y_{0}\left(\mathbf{G}_{\max }^{*}\right) & =w L+\sum_{i \in \mathbf{G}_{\max }^{*}}\left(\pi_{i}\left(\mathbf{G}_{\max }^{*}\right)-w z_{i}\right) \\
& \geq w L+\sum_{i \in \mathbf{G}^{*}}\left(\pi_{i}\left(\mathbf{G}_{\max }^{*}\right)-w z_{i}\right)
\end{aligned}
$$

where the inequality is a consequence of the fact that $\pi_{i}\left(\mathbf{G}_{\max }^{*}\right) \geq w z_{i}$ for all $i \in \mathbf{G}_{\max }^{*}$. Subtracting equation (B.17) from both sides of the above inequality leads to

$$
y_{0}\left(\mathbf{G}_{\max }^{*}\right)-y_{0}\left(\mathbf{G}^{*}\right) \geq \sum_{i \in \mathbf{G}^{*}} \pi_{i}\left(\mathbf{G}_{\max }^{*}\right)-\sum_{i \in \mathbf{G}^{*}} \pi_{i}\left(\mathbf{G}^{*}\right) .
$$

Recall that, when all production technologies are Leontief, the output of any customized firm $i$ satisfies $y_{i}=\alpha_{i} y_{0}$, where $y_{0}$ is the aggregate output and $\alpha_{i}$ is a constant that depends neither on prices nor quantities. Therefore,

$$
\begin{aligned}
y_{0}\left(\mathbf{G}_{\text {max }}^{*}\right)-y_{0}\left(\mathbf{G}^{*}\right) & \geq y_{0}\left(\mathbf{G}_{\text {max }}^{*}\right) \sum_{i \in \mathbf{G}^{*}} \alpha_{i} \mu_{i}\left(\mathbf{G}_{\text {max }}^{*}\right)-y_{0}\left(\mathbf{G}^{*}\right) \sum_{i \in \mathbf{G}^{*}} \alpha_{i} \mu_{i}\left(\mathbf{G}^{*}\right) \\
& \geq\left(y_{0}\left(\mathbf{G}_{\text {max }}^{*}\right)-y_{0}\left(\mathbf{G}^{*}\right)\right) \sum_{i \in \mathbf{G}^{*}} \alpha_{i} \mu_{i}\left(\mathbf{G}_{\text {max }}^{*}\right),
\end{aligned}
$$

where $\mu_{i}$ denotes firm $i$ 's markup and the second inequality is a consequence of Theorem 4 (a) and the fact that $\mathbf{G}^{*} \subseteq \mathbf{G}_{\max }^{*}$. Consequently,

$$
\left(y_{0}\left(\mathbf{G}_{\max }^{*}\right)-y_{0}\left(\mathbf{G}^{*}\right)\right)\left(1-\sum_{i \in \mathbf{G}^{*}} \alpha_{i} \mu_{i}\left(\mathbf{G}_{\max }^{*}\right)\right) \geq 0 .
$$

To establish that $y_{0}\left(\mathbf{G}_{\text {max }}^{*}\right) \geq y_{0}\left(\mathbf{G}^{*}\right)$, it is sufficient to show that the second term on the left-hand side of the above inequality is strictly positive. To this end, note that (B.18) can be rewritten as

$$
y_{0}\left(\mathbf{G}_{\max }^{*}\right)=w L+\sum_{i \in \mathbf{G}_{\max }^{*}} \alpha_{i} \mu_{i}\left(\mathbf{G}_{\max }^{*}\right) y_{0}\left(\mathbf{G}_{\max }^{*}\right)-\sum_{i \in \mathbf{G}_{\max }^{*}} w z_{i}>y_{0}\left(\mathbf{G}_{\max }^{*}\right) \sum_{i \in \mathbf{G}_{\max }^{*}} \alpha_{i} \mu_{i}\left(\mathbf{G}_{\max }^{*}\right)
$$

where the inequality follows from the fact that any full equilibrium is feasible and thus $L>\sum_{i \in \mathbf{G}_{\max }^{*}} z_{i}$. Dividing both sides of (B.20) by $y_{0}\left(\mathbf{G}_{\max }^{*}\right)$ and using the fact that $\mathbf{G}^{*} \subseteq \mathbf{G}_{\text {max }}^{*}$ establishes that

$$
1>\sum_{i \in \mathbf{G}_{\max }^{*}} \alpha_{i} \mu_{i}\left(\mathbf{G}_{\max }^{*}\right) \geq \sum_{i \in \mathbf{G}^{*}} \alpha_{i} \mu_{i}\left(\mathbf{G}_{\max }^{*}\right)
$$

This in juxtaposition with (B.19) guarantees that $y_{0}\left(\mathbf{G}_{\max }^{*}\right) \geq y_{0}\left(\mathbf{G}^{*}\right)$.

\section{Proof of Theorem 5}

Proof of part (a) The proof follows steps similar to those in the proof of Theorem 3(a). Let $A=$ $\left(A_{i j}\right)_{(j, i) \in \mathbf{G}}$ denote the vector of all pairwise customized productivities. Define the partition $\mathcal{S}(A)$ of the set of active firms $\mathbf{G}$ such that the parties in a supplier-customer pair $(j, i) \in \mathbf{G}$ belong to the same partition element if and only if the outside option in their pairwise bargaining game is strictly loose 
in equilibrium, that is, $p_{i j}^{a}<\bar{p}_{i j}$. Thus, the two firms belong to two different partition elements if $p_{i j}^{a}=\bar{p}_{i j}$.

Fix a supplier-customer pair of firms $(j, i) \in \mathbf{G}$ and all productivities $A_{-i j}$ other than $A_{i j}$. Define the sequence $0=A_{i j}^{(1)}<A_{i j}^{(2)}<\cdots<A_{i j}^{(m)}=\infty$ and the resulting collection of non-overlapping intervals $I_{k}=\left(A_{i j}^{(k)}, A_{i j}^{(k+1)}\right)$ such that

$$
\begin{array}{ll}
\mathcal{S}\left(A_{i j}, A_{-i j}\right)=\mathcal{S}\left(A_{i j}^{\prime}, A_{-i j}\right) & \text { if } A_{i j}, A_{i j}^{\prime} \in I_{k} \\
\mathcal{S}\left(A_{i j}, A_{-i j}\right) \neq \mathcal{S}\left(A_{i j}^{\prime}, A_{-i j}\right) & \text { if } A_{i j} \in I_{k}, A_{i j}^{\prime} \in I_{k}^{\prime} \text { and } k \neq k^{\prime} .
\end{array}
$$

Thus, the partition $\mathcal{S}\left(A_{i j}, A_{-i j}\right)$-and hence, the collection of pairwise bargaining problems in $\mathbf{G}$ for which the outside option constraint binds-remains unchanged for all $A_{i j}$ within the same interval $I_{k}$. Since there are only finitely many such intervals, if all markups in $S$ are weakly increasing in $A_{i j}$ in each interval $I_{k}$ for $1 \leq k<m$, then Lemma B.4 guarantees that they are weakly increasing in $A_{i j}$ over the entire $[0,1]$ interval as well. The fact that markups are monotonically increasing in $A_{i j}$ over interval $I_{k}$ then follows from equation (6) and steps similar to the proof of Theorem 3(a).

Proof of part (b) For a supplier-customer pair of active firms $(j, i) \in \mathbf{G}$, let $c_{\text {agg }}\left(A_{i j}\right)$ denote the equilibrium cost of transforming labor into one unit of the consumption good as a function of the productivity level $A_{i j}$, holding the set of active firms $\mathbf{G}$, the bargaining powers, and all other productivities constant. Similarly, let $y_{0}\left(A_{i j}\right)$ denote the equilibrium aggregate output as a function of $A_{i j}$. It is immediate that

$$
y_{0}\left(A_{i j}\right)=\frac{w}{c_{\mathrm{agg}}\left(A_{i j}\right)}\left(L-\sum_{k \in \mathbf{G}} z_{k}\right),
$$

where note that the wage $w$ does not depend on the productivity $A_{i j}$. Since all production functions are Leontief, the marginal cost $c_{\mathrm{agg}}\left(A_{i j}\right)$ of producing one unit of consumption good is decreasing in $A_{i j}$. Therefore, aggregate output $y_{0}\left(A_{i j}\right)$ is increasing in $A_{i j}$.

Proof of part (c) For a supplier-customer pair of active firms $(j, i) \in \mathbf{G}$, let $\pi_{k}\left(A_{i j}\right)$ denote the gross profit of firm $k \in \mathbf{G}$ as a function of the productivity level $A_{i j}$, holding the production network, the bargaining powers, and all other productivities constant. Since all production functions are Leontief,

$$
\pi_{k}\left(A_{i j}\right)=\alpha_{k}\left(A_{i j}\right) \mu_{k}\left(A_{i j}\right) y_{0}\left(A_{i j}\right)
$$

where $\alpha_{k}$ is a constant that only depends on productivities, $\mu_{k}$ is the markup of firm $k$, and $y_{0}$ is the aggregate output in the economy. The key observation is that, due to the assumption of Leontief technologies, $\alpha_{k}$ is independent of $A_{i j}$ as long as firm $k$ is not upstream to $j$, that is,

$$
\pi_{k}\left(A_{i j}\right)=\alpha_{k} \mu_{k}\left(A_{i j}\right) y_{0}\left(A_{i j}\right)
$$

In parts (a) and (b), we established that $\mu_{k}\left(A_{i j}\right)$ and $y_{0}\left(A_{i j}\right)$ are both increasing in $A_{i j}$. Therefore, as long as $k=j$ or $k$ is either downstream or horizontal to $j$, then an increase in $A_{i j}$ increases the profits of firm $k$. 
Proof of part (d) Consider a firm $k \in \mathbf{G}$ that is upstream to firm $j$. Since $\delta_{r j}=1$ for all firms $r$ such that $(r, j) \in \mathbf{G}$, $k$ 's markup $\mu_{k}$ is independent of the productivity $A_{i j}$ between the supplier-customer pair $(j, i) \in \mathbf{G}$. Therefore, $k$ 's profit as a function of $A_{i j}$ is given by

$$
\pi_{k}\left(A_{i j}\right)=\mu_{k} \alpha_{k}\left(A_{i j}\right) y_{0}\left(A_{i j}\right)
$$

where $y_{0}$ denotes the economy's aggregate output and $\alpha_{k}$ is independent of prices and quantities and is equal to the reciprocal of the product of customized productivities along the production chain from $k$ to the final good industry. As a result, $\alpha_{k}\left(A_{i j}\right)=\tilde{\alpha}_{k} / A_{i j}$, where $\tilde{\alpha}_{k}$ is a constant that is independent of $A_{i j}$. Consequently,

$$
\pi_{k}\left(A_{i j}\right)=\mu_{k} \tilde{\alpha}_{k} A_{i j}^{-1} y_{0}\left(A_{i j}\right) .
$$

Since all production technologies are Leontief and all firms use labor as an input for production,

$$
y_{0}\left(A_{i j}\right)=\frac{\zeta_{1}}{A_{i j}^{-1}+\zeta_{2}}
$$

for some $\zeta_{1}, \zeta_{2}>0$ that are independent of $A_{i j}$. The juxtaposition of the above two equations then guarantees that $\pi_{k}\left(A_{i j}\right)$ is decreasing in $A_{i j}$.

\section{Proof of Theorem 6}

Proof of part (a) Let $\bar{z}$ and $\underline{z}$ denote two vectors of fixed costs such that $\underline{z}_{i} \leq \bar{z}_{i}$ for all firms $i \in \mathcal{G}$. Define the mappings $\bar{\phi}, \underline{\phi}: 2^{\mathcal{G}} \rightarrow 2^{\mathcal{G}}$ as

$$
\begin{aligned}
& \bar{\phi}(\mathbf{G})=\left\{i \in \mathcal{G}: \pi_{i}(\{i\} \cup \mathbf{G}) \geq w \bar{z}_{i}\right\} \\
& \underline{\phi}(\mathbf{G})=\left\{i \in \mathcal{G}: \pi_{i}(\{i\} \cup \mathbf{G}) \geq w \underline{z}_{i}\right\} .
\end{aligned}
$$

It is immediate that the fixed points of $\bar{\phi}$ and $\underline{\phi}$ correspond to full equilibria of the economies with fixed costs given by $\bar{z}$ and $\underline{z}$, respectively. Furthermore, recall from the proof of Theorem 2 that $\bar{\phi}$ and $\phi$ satisfy Assumptions A.1 and A.2. Therefore, by Theorem A.1, both mappings have a greatest fixed point, which we denote by $\overline{\mathbf{G}}^{*}$ and $\underline{\mathbf{G}}^{*}$, respectively. Thus, $\overline{\mathbf{G}}^{*}$ and $\underline{\mathbf{G}}^{*}$ are also the greatest full equilibria of the economies with fixed costs $\bar{z}$ and $\underline{z}$, respectively.

On the other hand, the fact that $\underline{z}_{i} \leq \bar{z}_{i}$ for all $i \in \mathcal{G}$ implies that $\bar{\phi}(\mathbf{G}) \subseteq \underline{\phi}(\mathbf{G})$ for all $\mathbf{G} \subseteq \mathcal{G}$. Therefore, Corollary A.1 guarantees that $\overline{\mathbf{G}}^{*} \subseteq \underline{\mathbf{G}}^{*}$. This establishes that increasing the vector of fixed costs shrinks the set of active firms in the economy's greatest full equilibrium.

Proof of part (b) Let $\bar{z}$ and $\underline{z}$ denote two vectors of fixed costs such that $\underline{z}_{i} \leq \bar{z}_{i}$ for all $i \in \mathcal{G}$. Let $\overline{\mathbf{G}}^{*}$ and $\underline{G}^{*}$ denote the corresponding greatest full equilibria in the economies with fixed costs $\bar{z}$ and $\underline{z}$, respectively. In part (a), we already established that $\overline{\mathbf{G}}^{*} \subseteq \underline{\mathbf{G}}^{*}$. Furthermore, the household's budget constraint in the two economies is given by

$$
\begin{aligned}
& y_{0}(\bar{z})=w L+\sum_{k \in \overline{\mathbf{G}}^{*}}\left(\pi_{k}\left(\overline{\mathbf{G}}^{*}\right)-w \bar{z}_{k}\right) \\
& y_{0}(\underline{z})=w L+\sum_{k \in \underline{\mathbf{G}}^{*}}\left(\pi_{k}\left(\underline{\mathbf{G}}^{*}\right)-w \underline{z}_{k}\right) .
\end{aligned}
$$


Therefore,

$$
y_{0}(\underline{z})-y_{0}(\bar{z})=\sum_{k \in \underline{\mathbf{G}}^{*}}\left(\pi_{k}\left(\underline{\mathbf{G}}^{*}\right)-w \underline{z}_{k}\right)-\sum_{k \in \overline{\mathbf{G}}^{*}}\left(\pi_{k}\left(\overline{\mathbf{G}}^{*}\right)-w \bar{z}_{k}\right) .
$$

Since $\underline{G}^{*}$ is a full equilibrium, all active firms in the economy make positive net profits, i.e., $\pi_{k}\left(\underline{\mathbf{G}}^{*}\right) \geq$ $w z_{k}$ for all $k \in \underline{\mathbf{G}}^{*}$. Therefore,

$$
y_{0}(\underline{z})-y_{0}(\bar{z}) \geq \sum_{k \in \overline{\mathbf{G}}^{*}}\left(\pi_{k}\left(\underline{\mathbf{G}}^{*}\right)-w \underline{z}_{k}\right)-\sum_{k \in \overline{\mathbf{G}}^{*}}\left(\pi_{k}\left(\overline{\mathbf{G}}^{*}\right)-w \bar{z}_{k}\right) \geq \sum_{k \in \overline{\mathbf{G}}^{*}}\left(\pi_{k}\left(\underline{\mathbf{G}}^{*}\right)-\pi_{k}\left(\overline{\mathbf{G}}^{*}\right)\right),
$$

where the first inequality is a consequence of the fact that $\overline{\mathbf{G}}^{*} \subseteq \underline{\mathbf{G}}^{*}$ and the second inequality follows from the assumption that $\underline{z}_{k} \leq \bar{z}_{k}$. Since all production functions are Leontief, $\pi_{k}=\alpha_{k} \mu_{k} y_{0}$, where $\alpha_{k}>0$ is a constant that is independent of quantities and prices and $\mu_{k}$ is the markup of firm $k$. Hence:

$$
y_{0}(\underline{z})-y_{0}(\bar{z}) \geq \sum_{k \in \overline{\mathbf{G}}^{*}} \alpha_{k}\left(\mu_{k}\left(\underline{\mathbf{G}}^{*}\right) y_{0}(\underline{z})-\mu_{k}\left(\overline{\mathbf{G}}^{*}\right) y_{0}(\bar{z})\right) \geq\left(y_{0}(\underline{z})-y_{0}(\bar{z})\right) \sum_{k \in \overline{\mathbf{G}}^{*}} \alpha_{k} \mu_{k}\left(\underline{\mathbf{G}}^{*}\right) .
$$

The second inequality follows from the fact that $\mu_{k}\left(\overline{\mathbf{G}}^{*}\right) \leq \mu_{k}\left(\underline{\mathbf{G}}^{*}\right)$ for all $k \in \overline{\mathbf{G}}^{*}$ whenever $\overline{\mathbf{G}}^{*} \subseteq \underline{\mathbf{G}}^{*}$ (as established in Theorem 4(a)). Consequently,

$$
\left(y_{0}(\underline{z})-y_{0}(\bar{z})\right)\left(1-\sum_{k \in \overline{\mathbf{G}}^{*}} \alpha_{k} \mu_{k}\left(\underline{\mathbf{G}}^{*}\right)\right) \geq 0 .
$$

Therefore, as long as the second term on the left-hand side above is strictly positive, it is immediate that $y_{0}(\underline{z}) \geq y_{0}(\bar{z})$, which completes the proof. To establish this last claim, note that the household's budget constraint in (B.21) can be rewritten as

$$
y_{0}(\underline{z})=y_{0}(\underline{z}) \sum_{k \in \underline{\mathbf{G}}^{*}} \alpha_{k} \mu_{k}\left(\underline{\mathbf{G}}^{*}\right)+w\left(L-\sum_{k \in \underline{\mathbf{G}}^{*}} \underline{z}_{k}\right)>y_{0}(\underline{z}) \sum_{k \in \overline{\mathbf{G}}^{*}} \alpha_{k} \mu_{k}\left(\underline{\mathbf{G}}^{*}\right),
$$

where the inequality follows from the fact that the production network corresponding to any full equilibrium is feasible and that $\overline{\mathbf{G}}^{*} \subseteq \underline{\mathbf{G}}^{*}$. Consequently, $1-\sum_{k \in \overline{\mathbf{G}}^{*}} \alpha_{k} \mu_{k}\left(\underline{\mathbf{G}}^{*}\right)>0$, which in juxtaposition with (B.22) guarantees that $y_{0}(\underline{z}) \geq y_{0}(\bar{z})$.

Proof of part (c) Once again, let $\overline{\mathbf{G}}^{*}$ and $\underline{\mathbf{G}}^{*}$ denote the greatest full equilibria corresponding to fixed cost vectors $\bar{z} \geq \underline{z}$, respectively. In part (a), we established that $\overline{\mathbf{G}}^{*} \subseteq \underline{\mathbf{G}}^{*}$. By Theorem $4(\mathrm{a}), \mu_{k}\left(\overline{\mathbf{G}}^{*}\right) \leq$ $\mu_{k}\left(\underline{\mathbf{G}}^{*}\right)$ for all $k \in \overline{\mathbf{G}}^{*}$. Thus, all firm-level markups decrease in fixed costs. This observation also immediately implies that the same relationship also holds for firm-level profits. More specifically, recall that $\pi_{k}=\alpha_{k} \mu_{k} y_{0}$ for any firm $k$, where $\alpha_{k}$ only depends on productivities. Therefore, as fixed costs increase from $\underline{z}$ to $\bar{z}$, each firm $k \in \overline{\mathbf{G}}^{*}$ produces a smaller quantity (as established in part (b)), at a lower markup, while paying a weakly higher fixed cost. Thus, its net profit is decreasing in the vector of fixed costs. 


\section{Proof of Theorem 7}

Proof of part (a) Fix a supplier-customer pair of customized firms $(j, i) \in \mathcal{G}$ and consider two economies that are identical across all dimensions except for the productivity levels $\bar{A}_{i j}$ and $\underline{A}_{i j}$, where $\bar{A}_{i j} \geq \underline{A}_{i j}$. Denote the greatest full equilibria corresponding to the two economies by $\overline{\mathbf{G}}^{*}$ and $\underline{\mathbf{G}}^{*}$, respectively. Equivalently, $\overline{\mathbf{G}}^{*}$ and $\underline{\mathbf{G}}^{*}$ are, respectively, the greatest fixed points of mappings $\bar{\phi}, \underline{\phi}: 2^{\mathcal{G}} \rightarrow 2^{\mathcal{G}}$ defined as

$$
\begin{aligned}
& \bar{\phi}(\mathbf{G})=\left\{i \in \mathcal{G}: \pi_{i}\left(\{i\} \cup \mathbf{G} ; \bar{A}_{i j}\right) \geq w z_{i}\right\} \\
& \underline{\phi}(\mathbf{G})=\left\{i \in \mathcal{G}: \pi_{i}\left(\{i\} \cup \mathbf{G} ; \underline{A}_{i j}\right) \geq w z_{i}\right\} .
\end{aligned}
$$

Finally, suppose that both $i$ and $j$ are active in $\underline{\mathbf{G}}^{*}$ (i.e., $i, j \in \underline{\mathbf{G}}^{*}$ ) but $j$ has no active suppliers in $\underline{\mathbf{G}}^{*}$.

We first show that the set of active firms in the economy's greatest full equilibrium grows as we increase the productivity from $\underline{A}_{i j}$ to $\bar{A}_{i j}$, that is, $\underline{\mathbf{G}}^{*} \subseteq \overline{\mathbf{G}}^{*}$. To establish this, let $k$ denote an arbitrary firm in $\mathbf{G}^{*}$. By assumption, $k$ is either downstream, horizontal, or equal to $j$. Therefore, by Theorem 5(c), as long as the production network is held constant, $k$ 's profit is increasing in $A_{i j}$. In particular, $\pi_{k}\left(\mathbf{G}^{*} ; \bar{A}_{i j}\right) \geq \pi_{k}\left(\underline{\mathbf{G}}^{*} ; \underline{A}_{i j}\right)$. At the same time, the assumption that $k \in \mathbf{G}^{*}$ implies that $k$ makes nonnegative net profits in $\underline{\mathbf{G}}^{*}$, i.e., $\pi_{k}\left(\underline{\mathbf{G}}^{*} ; \underline{A}_{i j}\right) \geq w z_{k}$. Consequently, $\pi_{k}\left(\mathbf{G}^{*} ; \bar{A}_{i j}\right) \geq w z_{k}$, and as a result, $k \in \bar{\phi}\left(\underline{\mathbf{G}}^{*}\right)$ for all $k \in \underline{\mathbf{G}}^{*}$. We therefore just established that

$$
\underline{\mathbf{G}}^{*} \subseteq \bar{\phi}\left(\underline{\mathbf{G}}^{*}\right) .
$$

On the other hand, by part (c) of Theorem A.1, the greatest fixed point of $\bar{\phi}$ is given by

$$
\overline{\mathbf{G}}^{*}=\bigcup_{\mathbf{G} \in \overline{\mathcal{X}}} \mathbf{G},
$$

where $\overline{\mathcal{X}}=\{\mathbf{G} \subseteq \mathcal{G}: \mathbf{G} \subseteq \bar{\phi}(\mathbf{G})\}$. By (B.23), $\underline{\mathbf{G}}^{*} \in \overline{\mathcal{X}}$. Therefore, (B.24) implies that $\underline{\mathbf{G}}^{*} \subseteq \overline{\mathbf{G}}^{*}$.

Proof of part (b) We next show that an increase in productivity from $\underline{A}_{i j}$ to $\bar{A}_{i j}$ increases the profits of all already active firms under $\underline{A}_{i j}$. More specifically, we show that

$$
\pi_{k}\left(\overline{\mathbf{G}}^{*} ; \bar{A}_{i j}\right) \geq \pi_{k}\left(\underline{\mathbf{G}}^{*} ; \underline{A}_{i j}\right) \quad \text { for all } k \in \underline{\mathbf{G}}^{*} .
$$

To this end, recall that $\underline{G}^{*} \subseteq \overline{\mathbf{G}}^{*}$. Furthermore, the fact that $\overline{\mathbf{G}}^{*}$ is a full equilibrium implies that all firms in $\overline{\mathbf{G}}^{*}$ make positive net profits. Thus, $\sum_{k \in \overline{\mathbf{G}}^{*} \backslash \mathbf{G}^{*}}\left(\pi_{k}\left(\overline{\mathbf{G}}^{*} ; \bar{A}_{i j}\right)-w z_{k}\right) \geq 0$. Therefore, by Theorem 4(c), $\pi_{k}\left(\overline{\mathbf{G}}^{*} ; \bar{A}_{i j}\right) \geq \pi_{k}\left(\mathbf{G}^{*} ; \bar{A}_{i j}\right)$ for all $k \in \underline{\mathbf{G}}^{*}$. On the other hand, by assumption, $k \in \underline{\mathbf{G}}^{*}$ is either downstream, horizontal, or equal to $j$. Therefore, by Theorem $5(\mathbf{c}), \pi_{k}\left(\underline{\mathbf{G}}^{*} ; \bar{A}_{i j}\right) \geq \pi_{k}\left(\underline{\mathbf{G}}^{*} ; \underline{A}_{i j}\right)$ for all $k \in \underline{\mathbf{G}}^{*}$. Hence,

$$
\pi_{k}\left(\overline{\mathbf{G}}^{*} ; \bar{A}_{i j}\right) \geq \pi_{k}\left(\underline{\mathbf{G}}^{*} ; \bar{A}_{i j}\right) \geq \pi_{k}\left(\underline{\mathbf{G}}^{*} ; \underline{A}_{i j}\right),
$$

for all $k \in \underline{\mathbf{G}}^{*}$, thus establishing (B.25). 
Proof of part (c) Let $y_{0}\left(\underline{A}_{i j}\right)$ and $y_{0}\left(\bar{A}_{i j}\right)$ denote aggregate output under the two productivity levels. From the household's budget constraint, we have

$$
\begin{aligned}
& y_{0}\left(\bar{A}_{i j}\right)=w L+\sum_{k \in \overline{\mathbf{G}}^{*}}\left(\pi_{k}\left(\overline{\mathbf{G}}^{*} ; \bar{A}_{i j}\right)-w z_{k}\right) \\
& y_{0}\left(\underline{A}_{i j}\right)=w L+\sum_{k \in \underline{\mathbf{G}}^{*}}\left(\pi_{k}\left(\underline{\mathbf{G}}^{*} ; \underline{A}_{i j}\right)-w z_{k}\right) .
\end{aligned}
$$

Therefore,

$$
\begin{aligned}
y_{0}\left(\bar{A}_{i j}\right)-y_{0}\left(\underline{A}_{i j}\right) & =\sum_{k \in \overline{\mathbf{G}}^{*}}\left(\pi_{k}\left(\overline{\mathbf{G}}^{*} ; \bar{A}_{i j}\right)-w z_{k}\right)-\sum_{k \in \underline{\mathbf{G}}^{*}}\left(\pi_{k}\left(\underline{\mathbf{G}}^{*} ; \underline{A}_{i j}\right)-w z_{k}\right) \\
& \geq \sum_{k \in \underline{\mathbf{G}}^{*}}\left(\pi_{k}\left(\overline{\mathbf{G}}^{*} ; \bar{A}_{i j}\right)-w z_{k}\right)-\sum_{k \in \underline{\mathbf{G}}^{*}}\left(\pi_{k}\left(\underline{\mathbf{G}}^{*} ; \underline{A}_{i j}\right)-w z_{k}\right) \\
& =\sum_{k \in \underline{\mathbf{G}}^{*}}\left(\pi_{k}\left(\overline{\mathbf{G}}^{*} ; \bar{A}_{i j}\right)-\pi_{k}\left(\underline{\mathbf{G}}^{*} ; \underline{A}_{i j}\right)\right),
\end{aligned}
$$

where the inequality follows from the fact that $\overline{\mathbf{G}}^{*}$ is a full equilibrium, and as a result, all firms $k \in$ $\overline{\mathbf{G}}^{*} \backslash \underline{\mathbf{G}}^{*}$ make non-negative net profits, i.e., $\pi_{k}\left(\overline{\mathbf{G}}^{*} ; \bar{A}_{i j}\right) \geq w z_{k}$ for all $k \in \overline{\mathbf{G}}^{*} \backslash \underline{\mathbf{G}}^{*}$. Now (B.25) guarantees that the right-hand side of the above inequality is non-negative.

\section{Proof of Theorem 9}

The decomposition in (11) follows immediately from the definitions of $\left.\Delta \mathrm{GDP}\right|_{\underline{z} \text { fixed }}$ and $\left.\Delta \mathrm{GDP}\right|_{\overline{\mathrm{G}}^{*} \text { fixed }}$. It is therefore sufficient to show that both of these terms are non-negative. The fact that $\left.\Delta \mathrm{GDP}\right|_{\overline{\mathrm{G}}^{*} \text { fixed }} \geq 0$ is an immediate consequence of the observation that, holding all else (including the set of active firms) constant, a decrease in fixed costs can only increase aggregate output. To show that $\left.\Delta \mathrm{GDP}\right|_{\underline{z} \text { fixed }} \geq 0$, first note that Theorem 6(a) guarantees that the $\overline{\mathbf{G}}^{*} \subseteq \underline{\mathbf{G}}^{*}$. Furthermore, since $\underline{\mathbf{G}}^{*}$ is part of an equilibrium, all active firms $j \in \underline{\mathbf{G}}^{*}$ make non-negative net profits under $\underline{z}$. Hence,

$$
\sum_{j \in: \underline{\mathbf{G}}^{*} \backslash \overline{\mathbf{G}}^{*}}\left(\pi_{j}\left(\underline{\mathbf{G}}^{*}\right)-w \underline{z}_{j}\right) \geq 0 .
$$

Consequently, statement (b) of Theorem 4 implies that $\operatorname{GDP}\left(\underline{\mathbf{G}}^{*}, \underline{z}\right) \geq \operatorname{GDP}\left(\overline{\mathbf{G}}^{*}, \underline{z}\right)$. 


\section{References}

Acemoglu, Daron, Ufuk Akcigit, and William Kerr (2016), "Networks and the macroeconomy: An empirical exploration.” In National Bureau of Economic Research Macroeconomics Annual (Martin Eichenbaum and Jonathan Parker, eds.), volume 30, 276-335, University of Chicago Press.

Acemoglu, Daron and Pablo D. Azar (2020), “Endogenous production networks.” Econometrica, 88(1), 33-82.

Acemoglu, Daron, Vasco M. Carvalho, Asuman Ozdaglar, and Alireza Tahbaz-Salehi (2012), "The network origins of aggregate fluctuations.” Econometrica, 80(5), 1977-2016.

Acemoglu, Daron, Asuman Ozdaglar, and Alireza Tahbaz-Salehi (2015), "Systemic risk and stability in financial networks." American Economic Review, 105(2), 564-608.

Allen, Franklin and Douglas Gale (2000), “Financial contagion.” Journal of Political Economy, 108(1), $1-33$.

Baqaee, David R. (2018), “Cascading failures in production networks.” Econometrica, 86(5), 18191838.

Baqaee, David R. and Emmanuel Farhi (2020a), “Entry vs. rents.” NBER Working Paper No. 27140.

Baqaee, David R. and Emmanuel Farhi (2020b), "Productivity and misallocation in general equilibrium." Quarterly Journal of Economics, 135(1), 105-163.

Barrot, Jean-Noël and Julien Sauvagnat (2016), "Input specificity and the propagation of idiosyncratic shocks in production networks.” Quarterly Journal of Economics, 131(3), 1543-1592.

Berman, Abraham (1981), "Matrices and the linear complementarity problem." Linear Algebra and its Applications, 40, 249-256.

Bigio, Saki and Jennifer La'O (2020), “Distortions in production networks.” Quarterly Journal of Economics (forthcoming).

Bilbiie, Florin O., Fabio Ghironi, and Marc J. Melitz (2012), "Endogenous entry, product variety, and business cycles.” Journal of Political Economy, 120(2), 304-345.

Boehm, Christoph E., Aaron Flaaen, and Nitya Pandalai-Nayar (2019), "Input linkages and the transmission of shocks: Firm-level evidence from the 2011 Tohoku Earthquake." Review of Economics and Statistics, 101(1), 60-75.

Campbell, Jeffrey R. (1998), "Entry, exit, embodied technology, and business cycles." Review of Economic Dynamics, 1(2), 371-408.

Carvalho, Vasco M., Matthew Elliott, and John Spray (2020), “Network bottlenecks and market power.” Unpublished manuscript. 
Carvalho, Vasco M. and Xavier Gabaix (2013), “The Great Diversification and its undoing.” American Economic Review, 103(5), 1697-1727.

Carvalho, Vasco M. and Basile Grassi (2019), “Large firm dynamics and the business cycle.” American Economic Review, 109(4), 1375-1425.

Carvalho, Vasco M., Makoto Nirei, Yukiko Saito, and Alireza Tahbaz-Salehi (2017), "Supply chain disruptions: Evidence from the Great East Japan Earthquake.” Becker-Friedman Institute working paper 2017-01.

Carvalho, Vasco M. and Alireza Tahbaz-Salehi (2019), “Production networks: A primer.” Annual Review of Economics, 11(1), 635-663.

Carvalho, Vasco M. and Nico Voigtländer (2015), "Input diffusion and the evolution of production networks." NBER Working Paper No. 20025.

Chen, Hui (2010), "Macroeconomic conditions and the puzzles of credit spreads and capital structure." Journal of Finance, 65(6), 2171-2212.

Clementi, Gian Luca and Berardino Palazzo (2016), "Entry, exit, firm dynamics, and aggregate fluctuations." American Economic Journal: Macroeconomics, 8(3), 1-41.

Dunne, Timothy, Mark J. Roberts, and Larry Samuelson (1988), "Patterns of firm entry and exit in U.S. manufacturing industries.” The RAND Journal of Economics, 19(4), 495-515.

Elliott, Matthew, Benjamin Golub, and Matthew O. Jackson (2014), "Financial networks and contagion." American Economic Review, 104(10), 3115-3153.

Elliott, Matthew, Benjamin Golub, and Matthew V. Leduc (2020), "Supply network formation and fragility." Working paper.

Foster, Lucia, Cheryl Grim, and John Haltiwanger (2016), "Reallocation in the Great Recession: Cleansing or not?” Journal of Labor Economics, 34(S1), S293-S331.

Foster, Lucia, John Haltiwanger, and Chad Syverson (2008), "Reallocation, firm turnover, and efficiency: Selection on productivity or profitability?” American Economic Review, 98(1), 394-425.

Gabaix, Xavier (2011), “The granular origins of aggregate fluctuations.” Econometrica, 79(3), 733-772.

Hopenhayn, Hugo A. (1992), “Entry, exit, and firm dynamics in long run equilibrium.” Econometrica, 60, 1127-1150.

Huneeus, Federico (2019), "Production network dynamics and the propagation of shocks.” Working paper.

Jacobson, Tor and Erik von Schedvin (2015), "Trade credit and the propagation of corporate failure: An empirical analysis." Econometrica, 83(4), 1315-1371. 
Jones, Charles I. (2013), “Misallocation, economic growth, and input-output economics.” In Proceedings of Econometric Society World Congress (Daron Acemoglu, Manuel Arellano, and Eddie Dekel, eds.), 419-455, Cambridge University Press.

Jovanovic, Boyan (1982), “Selection and the evolution of industry.” Econometrica, 50(3), 649-670.

Kikkawa, Ayumu Ken, Glenn Magerman, and Emmanuel Dhyne (2020), "Imperfect competition in firm-to-firm trade." National Bank of Belgium Working paper No. 363.

Lim, Kevin (2018), "Endogenous production networks and the business cycle.” Working paper.

Liu, Ernest (2019), "Industrial policies in production networks.” Quarterly Journal of Economics, 134(4), 1883-1948.

Long, John B. and Charles I. Plosser (1983), “Real business cycles.” Journal of Political Economy, 91(1), 39-69.

Milgrom, Paul and John Roberts (1994), “Comparing equilibria.” The American Economic Review, 84(3), 441-459.

Oberfield, Ezra (2018), “A theory of input-output architecture.” Econometrica, 86(2), 559-589.

Osborne, Martin J. and Ariel Rubinstein (1990), Bargaining and Markets. Academic Press Inc.

Ravn, Morten O. and Harald Uhlig (2002), "On adjusting the Hodrick-Prescott filter for the frequency of observations.” The Review of Economics and Statistics, 84(2), 371-380.

Rubinstein, Ariel (1982), “Perfect equilibrium in a bargaining model.” Econometrica, 50(1), 97-109.

Samuelson, Paul A. (1951), "Abstract of a theorem concerning substitutability in open Leontief models.” In Activity Analysis of Production and Allocation (Tjalling C. Koopmans, ed.), volume 13, 142-146, Wiley, New York.

Shaked, Avner (1987), “Opting out: Bazaars versus “Hi Tech” markets.” Theoretical Economics Paper Series 159, Suntory and Toyota International Centres for Economics and Related Disciplines, LSE.

Taschereau-Dumouchel, Mathieu (2020), "Cascades and fluctuations in an economy with an endogenous production network." Working paper.

The White House (2012), "National strategy for global supply chain security." https:

//obamawhitehouse.archives.gov/sites/default/files/national_strategy_for_global_ supply_chain_security.pdf.

Tian, Can (2018), "Firm-level entry and exit dynamics over the business cycles." European Economic Review, 102, 298-326.

Topkis, Donald M. (1998), Supermodularity and Complementarity. Princeton University Press. 


\section{Online Appendix: Pairwise Bargaining}

In this appendix, we characterize the outcome of bargaining between a supplier-customer pair of active firms $(j, i) \in \mathbf{G}$, taking all other prices and the representative household's income as given. This characterization serves as the basis of the proofs of Theorems 1 and 10. Throughout, we suppress the dependence of variables on all other (customized and generic) prices $\left(\boldsymbol{p}_{-i j}^{a}, \boldsymbol{p}^{b}\right)$ for notational simplicity.

Let $\pi_{i}\left(p_{i j}^{a}\right)$ and $\pi_{j}\left(p_{i j}^{a}\right)$ denote, respectively, the customer's and supplier's profits (gross of fixed costs of operation) as functions of $p_{i j}^{a}$, holding all other prices $\left(\boldsymbol{p}_{-i j}^{a}, \boldsymbol{p}^{b}\right)$ fixed. We also use $c_{j}$ to denote the supplier's marginal cost. Note that $c_{j}$ does not depend on $p_{i j}^{a}$. It is immediate that there are positive gains from trade between $i$ and $j$ if and only if

$$
\pi_{i}\left(c_{j}\right) \geq 0 .
$$

If inequality (C.1) is violated, the bargaining game results in no agreement. In the proof of Theorem 10 , we show that the above inequality holds for any pair of active firms in any bargaining equilibrium. For the purposes of this appendix, however, we simply impose inequality (C.1) for the pair $(j, i) \in \mathbf{G}$, thus ensuring agreement in equilibrium.

To characterize the agreement price, we start with the following simple lemma:

Lemma C.1. $\pi_{i}\left(p_{i j}^{a}\right)$ is strictly decreasing in $p_{i j}^{a}$ and $\pi_{j}\left(p_{i j}^{a}\right)$ is strictly increasing in $p_{i j}^{a}$.

Proof. The monotonicity of $\pi_{i}$ is an immediate consequence of the fact that $i$ 's marginal cost is strictly increasing in any input price. The monotonicity of $j$ 's profit in its output price follows from Assumption $3^{\prime}$, which guarantees that all inputs in $i$ 's production function are gross complements.

Lemma C.2. If inequality (C.1) is satisfied, then equation $f_{i j}\left(p_{i j}\right)=0$ has at least one solution in the interval $\left[c_{j}, p_{i j}^{\dagger}\right]$, where $f_{i j}$ is given by equation (17) and $p_{i j}^{\dagger}$ is the unique price at which $\pi_{i}\left(p_{i j}^{\dagger}\right)=0$.

Proof. As a first observation, note that monotonicity of $\pi_{i}$ established in Lemma C.1 guarantees that there exists a unique $p_{i j}^{\dagger}$ such that $\pi_{i}\left(p_{i j}^{\dagger}\right)=0$. The same lemma also implies that, whenever inequality (C.1) is satisfied, then $p_{i j}^{\dagger} \geq c_{j}$. Therefore, $\left[c_{j}, p_{i j}^{\dagger}\right]$ is a non-empty interval.

Next, note that $f_{i j}\left(c_{j}\right)=\delta_{i j} \pi_{i}\left(c_{j}\right) \frac{\mathrm{d} \pi_{j}}{\mathrm{~d} p_{i j}}\left(c_{j}\right) \geq 0$, where the inequality follows from inequality (C.1) and monotonicity of $\pi_{j}$ established in Lemma C.1. Similarly, $f_{i j}\left(p_{i j}^{\dagger}\right)=(1-\delta) \pi_{j}\left(p_{i j}^{\dagger}\right) \frac{\mathrm{d} \pi_{i}}{\mathrm{~d} p_{i j}}\left(p_{i j}^{\dagger}\right) \leq 0$, where the inequality follows from the monotonicity of $\pi_{i}$ established in Lemma C.1. The two inequalities together imply that $f_{i j}\left(c_{j}\right) \geq 0 \geq f_{i j}\left(p_{i j}^{\dagger}\right)$. Therefore, equation $f_{i j}\left(p_{i j}\right)=0$ has at least one solution in $\left[c_{j}, p_{i j}^{\dagger}\right]$.

Lemma C.3. If inequality (C.1) is satisfied, then equation $f_{i j}\left(p_{i j}\right)=0$ has a unique solution in $\left[c_{j}, p_{i j}^{\dagger}\right]$, where $f_{i j}$ is given by equation (17).

Proof. The result is immediate if $c_{j}=p_{i j}^{\dagger}$. Therefore, in the rest of the proof we assume that the interval $\left[c_{j}, p_{i j}^{\dagger}\right]$ is not a singleton. We start with the observation that $f_{i j}\left(p_{i j}\right)=0$ for some $p_{i j} \in\left(c_{j}, p_{i j}^{\dagger}\right]$ if and only if $g_{i j}\left(p_{i j}\right)=1-1 / \delta_{i j}$, where

$$
g_{i j}\left(p_{i j}\right)=\left(\pi_{i} \frac{\mathrm{d} \pi_{j}}{\mathrm{~d} p_{i j}}\right) /\left(\pi_{j} \frac{\mathrm{d} \pi_{i}}{\mathrm{~d} p_{i j}}\right) .
$$


Since $g_{i j}\left(c_{j}\right)=-\infty$ and $g_{i j}\left(p_{i j}^{\dagger}\right)=0$, it is sufficient to show that $g_{i j}\left(p_{i j}\right)$ is strictly increasing over the interval $\left(c_{j}, p_{i j}^{\dagger}\right]$. Differentiating $g_{i j}$ with respect to $p_{i j}$ implies that

$$
\begin{aligned}
g_{i j}^{\prime}\left(p_{i j}\right) & =\left(\pi_{j} \frac{\mathrm{d} \pi_{i}}{\mathrm{~d} p_{i j}}\right)^{-2}\left(\pi_{i} \pi_{j} \frac{\mathrm{d}^{2} \pi_{j}}{\mathrm{~d} p_{i j}^{2}} \frac{\mathrm{d} \pi_{i}}{\mathrm{~d} p_{i j}}-\pi_{i} \pi_{j} \frac{\mathrm{d}^{2} \pi_{i}}{\mathrm{~d} p_{i j}^{2}} \frac{\mathrm{d} \pi_{j}}{\mathrm{~d} p_{i j}}-\pi_{i} \frac{\mathrm{d} \pi_{i}}{\mathrm{~d} p_{i j}}\left(\frac{\mathrm{d} \pi_{j}}{\mathrm{~d} p_{i j}}\right)^{2}+\pi_{j} \frac{\mathrm{d} \pi_{j}}{\mathrm{~d} p_{i j}}\left(\frac{\mathrm{d} \pi_{i}}{\mathrm{~d} p_{i j}}\right)^{2}\right) \\
& \geq \pi_{i}\left(\pi_{j} \frac{\mathrm{d} \pi_{i}}{\mathrm{~d} p_{i j}}\right)^{-2}\left(\pi_{j} \frac{\mathrm{d}^{2} \pi_{j}}{\mathrm{~d} p_{i j}^{2}} \frac{\mathrm{d} \pi_{i}}{\mathrm{~d} p_{i j}}-\pi_{j} \frac{\mathrm{d}^{2} \pi_{i}}{\mathrm{~d} p_{i j}^{2}} \frac{\mathrm{d} \pi_{j}}{\mathrm{~d} p_{i j}}-\frac{\mathrm{d} \pi_{i}}{\mathrm{~d} p_{i j}}\left(\frac{\mathrm{d} \pi_{j}}{\mathrm{~d} p_{i j}}\right)^{2}\right),
\end{aligned}
$$

where the inequality follows from the fact that $\pi_{j}$ is non-negative and increasing in $p_{i j}$ for all $p_{i j} \geq c_{j}$. Note that $\pi_{i}=\left(p_{r i}-c_{i}\right) y_{i}$ and $\pi_{j}=\left(p_{i j}-c_{j}\right) x_{i j}$, where $p_{r i}$ is the output price of firm $i, y_{i}$ is the quantity demanded from firm $i$ by its customer, and $x_{i j}$ is the quantity demanded by $i$ from $j$. Therefore, by Shephard's lemma,

$$
g_{i j}^{\prime}\left(p_{i j}\right) \geq \pi_{i} y_{i}^{3}\left(\frac{\mathrm{d} c_{i}}{\mathrm{~d} p_{i j}}\right)\left(\pi_{j} \frac{\mathrm{d} \pi_{i}}{\mathrm{~d} p_{i j}}\right)^{-2} h_{i j}\left(p_{i j}\right)
$$

where

$$
h_{i j}\left(p_{i j}\right)=\left(p_{i j}-c_{j}\right)^{2}\left[2\left(\frac{\mathrm{d}^{2} c_{i}}{\mathrm{~d} p_{i j}^{2}}\right)^{2}-\left(\frac{\mathrm{d} c_{i}}{\mathrm{~d} p_{i j}}\right)\left(\frac{\mathrm{d}^{3} c_{i}}{\mathrm{~d} p_{i j}^{3}}\right)\right]+\left(p_{i j}-c_{j}\right)\left(\frac{\mathrm{d} c_{i}}{\mathrm{~d} p_{i j}}\right)\left(\frac{\mathrm{d}^{2} c_{i}}{\mathrm{~d} p_{i j}^{2}}\right)+\left(\frac{\mathrm{d} c_{i}}{\mathrm{~d} p_{i j}}\right)^{2}
$$

Note that $\pi_{i} \geq 0$ for all $p_{i j} \in\left[c_{j}, p_{i j}^{\dagger}\right]$. Therefore, if $h_{i j}\left(p_{i j}\right) \geq 0$ for all $p_{i j} \in\left[c_{j}, p_{i j}^{\dagger}\right]$, it is then immediate that $g_{i j}\left(p_{i j}\right)$ is increasing in $p_{i j}$ and hence $f_{i j}\left(p_{i j}\right)=0$ has a unique solution in $\left[c_{j}, p_{i j}^{\dagger}\right]$. It is therefore sufficient to show that $h_{i j}\left(p_{i j}\right) \geq 0$. We consider two separate cases.

First, suppose the first term on the right-hand side of (C.2) is non-negative. In such a case, $h_{i j}\left(p_{i j}\right) \geq 0$ because the sum of the other two terms on the right-hand side of (C.2) is also non-negative:

$$
\left(p_{i j}-c_{j}\right)\left(\frac{\mathrm{d} c_{i}}{\mathrm{~d} p_{i j}}\right)\left(\frac{\mathrm{d}^{2} c_{i}}{\mathrm{~d} p_{i j}^{2}}\right)+\left(\frac{\mathrm{d} c_{i}}{\mathrm{~d} p_{i j}}\right)^{2}=\frac{1}{y_{i}} \frac{\mathrm{d} c_{i}}{\mathrm{~d} p_{i j}} \frac{\mathrm{d} \pi_{j}}{\mathrm{~d} p_{i j}} \geq 0,
$$

where the inequality follows from the monotonicity of $\pi_{j}$ established in Lemma C. 1 .

Next, suppose the first term on the right-hand side of (C.2) is strictly negative. Note that, in such a case, the right-hand side of (C.2) is concave in $c_{j}$ and is strictly positive when $c_{j}=p_{i j}$. Therefore, $h_{i j}\left(p_{i j}\right)$ is guaranteed to be non-negative for all $p_{i j} \geq c_{j}$ if the right-hand side of (C.2) is non-negative when $c_{j}=0$. Hence, the proof is complete if $\hat{h}_{i j}\left(p_{i j}\right) \geq 0$, where

$$
\hat{h}_{i j}\left(p_{i j}\right)=p_{i j}^{2}\left[2\left(\frac{\mathrm{d}^{2} c_{i}}{\mathrm{~d} p_{i j}^{2}}\right)^{2}-\left(\frac{\mathrm{d} c_{i}}{\mathrm{~d} p_{i j}}\right)\left(\frac{\mathrm{d}^{3} c_{i}}{\mathrm{~d} p_{i j}^{3}}\right)\right]+p_{i j}\left(\frac{\mathrm{d}^{2} c_{i}}{\mathrm{~d} p_{i j}^{2}}\right)\left(\frac{\mathrm{d} c_{i}}{\mathrm{~d} p_{i j}}\right)+\left(\frac{\mathrm{d} c_{i}}{\mathrm{~d} p_{i j}}\right)^{2} .
$$

Let $\sigma_{i}^{k j}$ denote the Morishima elasticity of substitution between inputs $j$ and $k$ in firm $i$ 's production technology, defined in footnote 23. Since firm $i$ 's cost function is homogenous of degree 1 , one can show that

$$
\frac{\mathrm{d}^{2} c_{i}}{\mathrm{~d} p_{i j}^{2}} / \frac{\mathrm{d} c_{i}}{\mathrm{~d} p_{i j}}=-\frac{1}{p_{i j} c_{i}} \sum_{k \neq j} \sigma_{i}^{k j} p_{i k} \frac{\mathrm{d} c_{i}}{\mathrm{~d} p_{i k}}
$$


where the sum is over all inputs $k \neq j$ (including labor input) of firm $i$ and we are using the fact that

$$
\sigma_{i}^{k j}=p_{i j}\left(\frac{\mathrm{d}^{2} c_{i}}{\mathrm{~d} p_{i j} \mathrm{~d} p_{i k}} / \frac{\mathrm{d} c_{i}}{\mathrm{~d} p_{i k}}-\frac{\mathrm{d}^{2} c_{i}}{\mathrm{~d} p_{i j}^{2}} / \frac{\mathrm{d} c_{i}}{\mathrm{~d} p_{i j}}\right) .
$$

Differentiating equation (C.4) with respect to $p_{i j}$ and rearranging terms implies that

$$
\begin{aligned}
p_{i j}^{2}\left(2 \frac{\mathrm{d}^{2} c_{i}}{\mathrm{~d} p_{i j}^{2}}-\frac{\mathrm{d} c_{i}}{\mathrm{~d} p_{i j}} \frac{\mathrm{d}^{3} c_{i}}{\mathrm{~d} p_{i j}^{3}}\right) /\left(\frac{\mathrm{d} c_{i}}{\mathrm{~d} p_{i j}}\right)^{2}= & \frac{p_{i j}}{c_{i}} \sum_{k \neq j} p_{i k} \frac{\mathrm{d} c_{i}}{\mathrm{~d} p_{i k}} \frac{\mathrm{d} \sigma_{i}^{k j}}{\mathrm{~d} p_{i j}}+\frac{1}{c_{i}} \sum_{k \neq j}\left(\sigma_{i}^{k j}\right)^{2} p_{i k} \frac{\mathrm{d} c_{i}}{\mathrm{~d} p_{i k}} \\
& -\left(\frac{1}{c_{i}}+\frac{p_{i j}}{c_{i}^{2}} \frac{\mathrm{d} c_{i}}{\mathrm{~d} p_{i j}}\right) \sum_{k \neq j} \sigma_{i}^{k j} p_{i k} \frac{\mathrm{d} c_{i}}{\mathrm{~d} p_{i k}} .
\end{aligned}
$$

Plugging (C.4) and (C.5) into the expression for $\hat{h}_{i j}\left(p_{i j}\right)$ in equation (C.3) implies that

$$
\begin{gathered}
\hat{h}\left(p_{i j}\right)=\left(\frac{\mathrm{d} c_{i}}{\mathrm{~d} p_{i j}}\right)^{2}\left[\sum_{k \neq j}\left(1-\sigma_{i}^{k j}\right)^{2} \frac{\mathrm{d} \log c_{i}}{\mathrm{~d} \log p_{i k}}+\frac{\mathrm{d} \log c_{i}}{\mathrm{~d} \log p_{i j}} \sum_{k \neq j}\left(1-\sigma_{i}^{k j}\right) \frac{\mathrm{d} \log c_{i}}{\mathrm{~d} \log p_{i k}}\right. \\
\left.+\left(\frac{\mathrm{d} \log c_{i}}{\mathrm{~d} \log p_{i j}}\right)^{2}+\sum_{k \neq j} \frac{\mathrm{d} \log c_{i}}{\mathrm{~d} \log p_{i k}} \frac{\mathrm{d} \sigma_{i}^{k j}}{\mathrm{~d} \log p_{i j}}\right] .
\end{gathered}
$$

The fact that $i$ 's marginal cost is increasing in all input prices, alongside the assumption that $\sigma_{j k} \leq 1$, guarantees that the second and third terms on the right-hand side of (C.6) are non-negative, while inequality (14) guarantees that the sum of the first and the last term is also non-negative. Together, these observations imply that $\hat{h}_{i j}\left(p_{i j}\right) \geq 0$ for all $p_{i j}$, which completes the proof.

Using the previous lemmas, our next result characterizes the outcome of pairwise bargaining between $i$ and $j$ as $\eta \rightarrow 1$, taking all other prices and the household's income as given.

Proposition C.1. Suppose inequality (C.1) is satisfied. Then, $i$ and $j$ reach an agreement in the pairwise bargaining game. Furthermore, as $\eta \rightarrow 1$, the agreement price converges to

$$
p_{i j}^{a}=\min \left\{\hat{p}_{i j}, \bar{p}_{i j}\right\}
$$

where $\hat{p}_{i j}$ is the unique solution to $f_{i j}\left(\hat{p}_{i j}\right)=0$ in the interval $\left[c_{j}, p_{i j}^{\dagger}\right]$ and $\bar{p}_{i j}=p_{j}^{b} A_{i j} / B_{i j}$.

Proof. The assumption that inequality (C.1) is satisfied guarantees that there are positive gains from trade. Therefore, the two firms reach an agreement in the SPNE of the pairwise bargaining game.

Next, we characterize the agreement price. Following standard arguments (say, as in Osborne and Rubinstein (1990)), any SPNE of the pairwise bargaining game has a stationary structure, with firms $i$ and $j$ respectively offering $p_{i j}^{\prime}$ and $p_{i j}^{\prime \prime}$ in any subgame that they have the opportunity to propose a price. Furthermore, the equilibrium price pair $\left(p_{i j}^{\prime}, p_{i j}^{\prime \prime}\right)$ satisfies the following indifference conditions:

$$
\begin{aligned}
& \pi_{j}\left(p_{i j}^{\prime}\right)=\eta \delta_{i j} \pi_{j}\left(p_{i j}^{\prime \prime}\right)+\eta\left(1-\delta_{i j}\right) \pi_{j}\left(p_{i j}^{\prime}\right) \\
& \pi_{i}\left(p_{i j}^{\prime \prime}\right)=\max \left\{\eta \delta_{i j} \pi_{i}\left(p_{i j}^{\prime \prime}\right)+\eta\left(1-\delta_{i j}\right) \pi_{i}\left(p_{i j}^{\prime}\right), \pi_{i}\left(\bar{p}_{i j}\right)\right\},
\end{aligned}
$$

where recall that firm $i$ always has the outside option of using the generic variant of the input produced by firm $j$ and obtain a profit of $\pi_{i}\left(\bar{p}_{i j}\right)$. 
To determine the agreement price as $\eta \rightarrow 1$, note that the first indifference condition in (C.8) guarantees that $\lim _{\eta \rightarrow 1} p_{i j}^{\prime}-p_{i j}^{\prime \prime}=0$. Therefore, it is sufficient to determine $p_{i j}^{\prime \prime}$ as $\eta \rightarrow 1$. Furthermore, since $\pi_{i}$ is monotone (Lemma C.1), the second indifference condition in (C.8) guarantees that the agreement price can never exceed $\bar{p}_{i j}$. Therefore, in what follows, we determine the agreement price while ignoring the customer's outside option and then impose the condition that the agreement price cannot exceed $\bar{p}_{i j}$. Solving for $p_{i j}^{\prime}$ from the two indifference conditions in (C.8) and equating the solutions implies that

$$
\pi_{j}^{-1}\left(\frac{\eta \delta_{i j}}{1-\eta\left(1-\delta_{i j}\right)} \pi_{j}\left(p_{i j}^{\prime \prime}\right)\right)=\pi_{i}^{-1}\left(\frac{1-\eta \delta_{i j}}{\eta\left(1-\delta_{i j}\right)} \pi_{i}\left(p_{i j}^{\prime \prime}\right)\right),
$$

where recall from Lemma C. 1 that $\pi_{i}$ and $\pi_{j}$ are strictly monotone in $p_{i j}$ and are hence invertible. It is then easy to verify that dividing both sides of the above equation by $1-\eta$ and taking the limit as $\eta \rightarrow 1$ reduces the above equation to equation (17). Therefore, as long as the customer's outside option does not bind, the price in the subgame perfect Nash equilibrium of the bargaining game between $i$ and $j$ is given by the solution to the equation $f_{i j}\left(p_{i j}\right)=0$, where $f_{i j}$ is defined in (17). Crucially, Lemma C.3 guarantees that this equation has a unique solution $\hat{p}_{i j}$ in the interval $\left[c_{j}, p_{i j}^{\dagger}\right]$. Imposing the condition that the agreement price cannot exceed the $\bar{p}_{i j}$ then establishes (C.7).

\section{Online Appendix: Omitted Proofs}

\section{Proof of Theorem 8}

Denote the greatest full equilibria corresponding to productivity levels $\bar{A}_{i j}$ and $\underline{A}_{i j}$ by $\overline{\mathbf{G}}^{*}$ and $\underline{\mathbf{G}}^{*}$, respectively. By definition, $\overline{\mathbf{G}}^{*}$ and $\underline{\mathbf{G}}^{*}$ are the greatest fixed points of mappings

$$
\begin{aligned}
& \bar{\phi}(\mathbf{G})=\left\{i \in \mathcal{G}: \pi_{i}\left(\{i\} \cup \mathbf{G} ; \bar{A}_{i j}\right) \geq w z_{i}\right\} \\
& \underline{\phi}(\mathbf{G})=\left\{i \in \mathcal{G}: \pi_{i}\left(\{i\} \cup \mathbf{G} ; \underline{A}_{i j}\right) \geq w z_{i}\right\},
\end{aligned}
$$

respectively. Also define $\tilde{\theta}=\pi_{k_{1}}\left(\overline{\mathbf{G}}^{*} \cup\left\{k_{1}\right\}, \bar{A}_{i j}\right) / w z_{k_{1}}$ and $\epsilon=\underline{A}_{i j} / \bar{A}_{i j}$. By assumption, $\tilde{\theta}<1$ and $\epsilon<1$. We start by stating and proving a series of lemmas.

Lemma D.1. For all $s \geq 1$,

$$
\underline{\phi}^{(s-1)}\left(\overline{\mathbf{G}}^{*}\right) \subseteq \underline{\phi}^{(s)}\left(\overline{\mathbf{G}}^{*}\right) .
$$

Proof. We prove the lemma using an inductive argument. To establish the induction base, note that, by assumption, $\pi_{k}\left(\overline{\mathbf{G}}^{*}, \underline{A}_{i j}\right) \geq w z_{k}$ for all $k \in \overline{\mathbf{G}}^{*}$. Therefore, $\overline{\mathbf{G}}^{*} \subseteq \underline{\phi}\left(\overline{\mathbf{G}}^{*}\right)$. Next, as the induction hypothesis, suppose (D.1) holds for some $s$. Since $\underline{\phi}^{(s)}\left(\overline{\mathbf{G}}^{*}\right) \subseteq \underline{\phi}^{(s)}\left(\overline{\mathbf{G}}^{*}\right)$ and the mapping $\underline{\phi}$ satisfies Assumption A.1 (established as part of proof of Theorem 2), we have $\underline{\phi}^{(s)}\left(\overline{\mathbf{G}}^{*}\right) \subseteq \underline{\phi}\left(\underline{\phi}^{(s)}\left(\overline{\mathbf{G}}^{*}\right) \cup\right.$ $\left.\underline{\phi}^{(s-1)}\left(\overline{\mathbf{G}}^{*}\right)\right)=\underline{\phi}^{s+1}\left(\overline{\mathbf{G}}^{*}\right)$, thus completing the inductive argument.

Lemma D.2. For any $\epsilon<1$, there exists $\vartheta(\epsilon)<1$ such that if $\tilde{\theta}>\vartheta(\epsilon)$, then $\underline{\phi}\left(\overline{\mathbf{G}}^{*}\right)=\overline{\mathbf{G}}^{*} \cup\left\{k_{1}\right\}$.

Proof. By Theorem 5(c), $\pi_{k}\left(\overline{\mathbf{G}}^{*} \cup\{k\}, \underline{A}_{i j}\right) \leq \pi_{k}\left(\overline{\mathbf{G}}^{*} \cup\{k\}, \bar{A}_{i j}\right)$ for any firm $k$ that is either downstream or horizontal to $i$. Therefore, if $k \in \underline{\phi}\left(\overline{\mathbf{G}}^{*}\right) \backslash \overline{\mathbf{G}}^{*}$, then $k$ has to be upstream to $j$. But since the sequence of 
firms $\left(k_{1}, \ldots, k_{s}, j\right)$ each have a single supplier, the only firm upstream to $j$ that can make non-negative profits is $k_{1}$, therefore establishing that $\phi\left(\overline{\mathbf{G}}^{*}\right) \subseteq \overline{\mathbf{G}}^{*} \cup\left\{k_{1}\right\}$.

Next, note that since $k_{1}$ is upstream to $j$, Theorem 5 (d) implies that $\pi_{k_{1}}\left(\overline{\mathbf{G}}^{*} \cup\left\{k_{1}\right\}, A_{i j}\right)$ is strictly decreasing in $A_{i j}$. Since $\pi_{k_{1}}\left(\overline{\mathbf{G}}^{*} \cup\left\{k_{1}\right\}, \bar{A}_{i j}\right)=\tilde{\theta} w z_{k_{1}}$, for any $\epsilon<1$, there exists a $\vartheta(\epsilon)$ close enough to 1 such that $\pi_{k_{1}}\left(\overline{\mathbf{G}}^{*} \cup\left\{k_{1}\right\}, \underline{A}_{i j}\right) \geq w z_{k_{1}}$ if $\tilde{\theta}>\vartheta(\epsilon)$. Consequently, $k_{1} \in \underline{\phi}\left(\overline{\mathbf{G}}^{*}\right)$. Therefore, by equation (D.1), $\overline{\mathbf{G}}^{*} \cup\left\{k_{1}\right\} \subseteq \underline{\phi}\left(\mathbf{G}^{*}\right)$.

Lemma D.3. Suppose $\tilde{\theta}>\vartheta(\epsilon)$, where $\vartheta(\epsilon)$ is defined in Lemma D.2. Then, there exist $\tilde{z}_{k_{0}}$ such that if $z_{k_{0}}<\tilde{z}_{k_{0}}$, then $y_{0}\left(\overline{\mathbf{G}}^{*} \cup\left\{k_{0}, k_{1}\right\}, \underline{A}_{i j}\right) \leq y_{0}\left(\underline{\phi}^{(2)}\left(\overline{\mathbf{G}}^{*}\right), \underline{A}_{i j}\right)$.

Proof. The lemma immediately follows if $\overline{\mathbf{G}}^{*} \cup\left\{k_{0}, k_{1}\right\}=\underline{\phi}^{(2)}\left(\overline{\mathbf{G}}^{*}\right)$. We therefore assume that $\overline{\mathbf{G}}^{*} \cup$ $\left\{k_{0}, k_{1}\right\} \neq \underline{\phi}^{(2)}\left(\overline{\mathbf{G}}^{*}\right)$. In the previous lemma, we already established that, if $\tilde{\theta}>\vartheta(\epsilon)$, then $\underline{\phi}\left(\overline{\mathbf{G}}^{*}\right)=$ $\overline{\mathbf{G}}^{*} \cup\left\{k_{1}\right\}$. Since $k_{0}$ is the only potential customized supplier of $k_{1}$, for small enough value of $z_{k_{0}}$, $k_{0} \in \underline{\phi}^{(2)}\left(\overline{\mathbf{G}}^{*}\right)$. This, coupled with (D.1) implies that $\overline{\mathbf{G}}^{*} \cup\left\{k_{0}, k_{1}\right\} \subsetneq \underline{\phi}^{(2)}\left(\overline{\mathbf{G}}^{*}\right)$.

Consider an arbitrary $k \in \underline{\phi}^{(2)}\left(\overline{\mathbf{G}}^{*}\right) \backslash \overline{\mathbf{G}}^{*} \cup\left\{k_{0}, k_{1}\right\}$. Since $\underline{\phi}^{(2)}\left(\overline{\mathbf{G}}^{*}\right) \subseteq \underline{\phi}^{(3)}\left(\overline{\mathbf{G}}^{*}\right)$-guaranteed by Lemma D.2- $\pi_{k}\left(\underline{\phi}^{(2)}\left(\overline{\mathbf{G}}^{*}\right), \underline{A}_{i j}\right) \geq w z_{k}$. Hence,

$$
\sum_{k \in \underline{\phi}^{(2)}\left(\overline{\mathbf{G}}^{*}\right) \backslash \overline{\mathbf{G}}^{*} \cup\left\{k_{0}, k_{1}\right\}}\left(\pi_{k}\left(\underline{\phi}^{(2)}\left(\overline{\mathbf{G}}^{*}\right), \underline{A}_{i j}\right)-w z_{k}\right) \geq 0 .
$$

Therefore, Theorem 4 (b) guarantees that $y_{0}\left(\overline{\mathbf{G}}^{*} \cup\left\{k_{0}, k_{1}\right\}, \underline{A}_{i j}\right) \leq y_{0}\left(\underline{\phi}^{(2)}\left(\overline{\mathbf{G}}^{*}\right), \underline{A}_{i j}\right)$.

Lemma D.4. There exist constants $\vartheta_{2}<1, \tilde{A}_{k_{1} k_{0}}$, and $\tilde{z}_{k_{0}}$ such that if $\tilde{\theta} \in\left(\vartheta_{2}, 1\right), A_{k_{1} k_{0}}>\tilde{A}_{k_{1} k_{0}}$, and $z_{k_{0}}<\tilde{z}_{k_{0}}$, then $y_{0}\left(\overline{\mathbf{G}}^{*}, \bar{A}_{i j}\right) \leq y_{0}\left(\overline{\mathbf{G}}^{*} \cup\left\{k_{0}, k_{1}\right\}, \underline{A}_{i j}\right)$.

Proof. We start with the observation that since all production functions are Leontief and $\delta_{k_{2} k_{1}}=1$,

$$
\pi_{k_{1}}\left(\overline{\mathbf{G}}^{*} \cup\left\{k_{1}\right\}, \bar{A}_{i j}\right)=c_{k_{1}}\left(B_{k_{2} k_{1}}^{-1}-A_{k_{2} k_{1}}^{-1}\right) \frac{y_{0}\left(\overline{\mathbf{G}}^{*} \cup\left\{k_{1}\right\}, \bar{A}_{i j}\right)}{A^{d} \bar{A}_{i j} A^{u}},
$$

where $c_{k_{1}}$ is the marginal cost of firm $k_{1}$ and $A^{u}$ and $A^{d}$ are positive constants that only depend on the productivities of active customized firms that are upstream and downstream to the pair $(j, i)$ in $\overline{\mathbf{G}}^{*}$, respectively, as well as generic productivities. Next, note that aggregate output is given by

$$
y_{0}\left(\overline{\mathbf{G}}^{*} \cup\left\{k_{1}\right\}, \bar{A}_{i j}\right)=\frac{L-z_{k_{1}}-\sum_{k \in \overline{\mathbf{G}}^{*}} z_{k}}{\tilde{A}^{-1}+\left(A^{d} \bar{A}_{i j} A^{u} A_{k_{2} k_{1}} w / c_{k_{1}}\right)^{-1}},
$$

where $\tilde{A}$ is a positive constant that only depends on productivities of active customized firms that are either downstream or horizontal to $j$ and the generic productivities. Solving for $z_{k_{1}}$ from the above equations and using the definition of $\tilde{\theta}$ imply that

$$
\frac{z_{k_{1}}}{L-\sum_{k \in \overline{\mathbf{G}}^{*}} z_{k}}=\frac{c_{k_{1}}\left(B_{k_{2} k_{1}}^{-1}-A_{k_{2} k_{1}}^{-1}\right)}{c_{k_{1}}\left(B_{k_{2} k_{1}}^{-1}-(1-\tilde{\theta}) A_{k_{1} k_{2}}^{-1}\right)+\tilde{\theta} w A^{d} \bar{A}_{i j} A^{u} \tilde{A}^{-1}} .
$$

On the other hand, using expressions similar to equation (D.2) for $y_{0}\left(\overline{\mathbf{G}}^{*}, \bar{A}_{i j}\right)$ and $y_{0}\left(\overline{\mathbf{G}}^{*} \cup\right.$ $\left.\left\{k_{0}, k_{1}\right\}, \underline{A}_{i j}\right)$ implies that

$$
\frac{y_{0}\left(\overline{\mathbf{G}}^{*} \cup\left\{k_{0}, k_{1}\right\}, \underline{A}_{i j}\right)}{y_{0}\left(\overline{\mathbf{G}}^{*}, \bar{A}_{i j}\right)}=\left(\frac{L-z_{k_{1}}-z_{k_{0}}-\sum_{k \in \overline{\mathbf{G}}^{*}} z_{k}}{L-\sum_{k \in \overline{\mathbf{G}}^{*}} z_{k}}\right)\left(\frac{\tilde{A}^{-1}+\left(A^{d} \bar{A}_{i j} A^{u} B_{k_{2} k_{1}} w / c_{k_{1}}\right)^{-1}}{\tilde{A}^{-1}+\left(A^{d} \underline{A}_{i j} A^{u} A_{k_{2} k_{1}} A_{k_{1} k_{0}} w / c_{k_{0}}\right)^{-1}}\right) .
$$


As a result,

$$
\lim _{A_{k_{1} k_{0}} \rightarrow \infty} \lim _{z_{k_{0}} \rightarrow 0} \frac{y_{0}\left(\overline{\mathbf{G}}^{*} \cup\left\{k_{0}, k_{1}\right\}, \underline{A}_{i j}\right)}{y_{0}\left(\overline{\mathbf{G}}^{*}, \bar{A}_{i j}\right)}=\left(1-\frac{z_{k_{1}}}{L-\sum_{k \in \overline{\mathbf{G}}^{*}} z_{k}}\right)\left(1+\frac{c_{k_{1}} B_{k_{2} k_{1}}^{-1}}{w A^{d} \bar{A}_{i j} A^{u} \tilde{A}^{-1}}\right) .
$$

The proof is complete once we show that there exists $\vartheta_{2}<1$ such that if $\tilde{\theta} \in\left(\vartheta_{2}, 1\right)$, the right-hand side of the above equation is greater than or equal to 1 . Equivalently, it is sufficient to show that for $\tilde{\theta}$ sufficiently close to 1 ,

$$
\frac{z_{k_{1}}}{L-\sum_{k \in \overline{\mathbf{G}}^{*}} z_{k}} \leq \frac{c_{k_{1}} B_{k_{2} k_{1}}^{-1}}{c_{k_{1}} B_{k_{2} k_{1}}^{-1}+w \tilde{A}^{-1} A^{d} \bar{A}_{i j} A^{u}} .
$$

Replacing for the right-hand side of the above inequality from equation (D.3) immediately implies that the inequality is satisfied for $\tilde{\theta}$ close enough to 1 .

Proof of part (a) By Lemma D.2, there exists $\vartheta(\epsilon)<1$ such that if $\tilde{\theta}>\vartheta(\epsilon)$, then $\overline{\mathbf{G}}^{*} \subsetneq \phi\left(\overline{\mathbf{G}}^{*}\right)$. Thus, for any $\underline{A}_{i j}<\bar{A}_{i j}$ such that $\pi_{k}\left(\overline{\mathbf{G}}^{*}, \underline{A}_{i j}\right) \geq w z_{k}$ for all $k \in \overline{\mathbf{G}}^{*}$, there exists $\theta<1$, such that if $\pi_{k_{1}}\left(\overline{\mathbf{G}}^{*} \cup\right.$ $\left.\left\{k_{1}\right\}, \bar{A}_{i j}\right)>\theta w z_{k_{1}}$, then $\overline{\mathbf{G}}^{*} \subsetneq \underline{\phi}\left(\overline{\mathbf{G}}^{*}\right)$. Furthermore, by equation (D.1), $\underline{\phi}\left(\overline{\mathbf{G}}^{*}\right) \subseteq \underline{\phi}^{(2)}\left(\overline{\mathbf{G}}^{*}\right)$, which implies that $\underline{\phi}\left(\overline{\mathbf{G}}^{*}\right) \in \underline{\mathcal{X}}$, where $\underline{\mathcal{X}}=\{\mathbf{G} \subseteq \mathcal{G}: \mathbf{G} \subseteq \underline{\phi}(\mathbf{G})\}$. Therefore, $\underline{\phi}\left(\overline{\mathbf{G}}^{*}\right) \subseteq \bigcup_{\mathbf{G} \in \mathcal{X}} \mathbf{G}=\underline{\mathbf{G}}^{*}$, where the equality follows from part (c) of Theorem A.1. Thus, it must be the case that $\overline{\mathbf{G}}^{*} \subsetneq \underline{\mathbf{G}}^{*}$.

Proof of part (b) Let $\vartheta_{3}=\max \left\{\vartheta_{1}(\epsilon), \vartheta_{2}\right\}<1$, where $\vartheta_{1}(\epsilon)$ and $\vartheta_{2}$ are defined in Lemmas D.3 and D.4, respectively. The juxtaposition of Lemmas D.3 and D.4 implies that there exist $\tilde{A}_{k_{1} k_{0}}$ and $\tilde{z}_{k_{0}}$ such that as long $\tilde{\theta} \in\left(\vartheta_{3}, 1\right), A_{k_{1} k_{0}}>\tilde{A}_{k_{1} k_{0}}$, and $z_{k_{0}}<\tilde{z}_{k_{0}}$, then

$$
y_{0}\left(\overline{\mathbf{G}}^{*}, \bar{A}_{i j}\right) \leq y_{0}\left(\overline{\mathbf{G}}^{*} \cup\left\{k_{0}, k_{1}\right\}, \underline{A}_{i j}\right) \leq y_{0}\left(\underline{\phi}^{(2)}\left(\overline{\mathbf{G}}^{*}\right), \underline{A}_{i j}\right) .
$$

On the other hand, by (D.1), $\underline{\phi}^{(2)}\left(\overline{\mathbf{G}}^{*}\right) \in \underline{\mathcal{X}}=\{\mathbf{G} \subseteq \mathcal{G}: \mathbf{G} \subseteq \underline{\phi}(\mathbf{G})\}$. Thus, by part (c) of Theorem A.1, $\underline{\phi}^{(2)}\left(\overline{\mathbf{G}}^{*}\right) \subseteq \underline{\mathbf{G}}^{*}$. Furthermore, since $\underline{\mathbf{G}}^{*}$ is a fixed point of $\underline{\phi}$, all firms $k \in \underline{\mathbf{G}}^{*}$ make non-negative profits under productivity $\underline{A}_{i j}$, and in particular, $\sum_{k \in \underline{\mathbf{G}}^{*} \backslash \underline{\phi}^{(2)}\left(\overline{\mathbf{G}}^{*}\right)}\left(\bar{\pi}_{k}\left(\underline{\mathbf{G}}^{*}, \underline{A}_{i j}\right)-w z_{k}\right) \geq 0$. Therefore, part (b) of Theorem 4 implies that

$$
y_{0}\left(\underline{\phi}^{(2)}\left(\overline{\mathbf{G}}^{*}\right), \underline{A}_{i j}\right) \leq y_{0}\left(\underline{\mathbf{G}}^{*}, \underline{A}_{i j}\right) .
$$

The juxtaposition of the above inequality with (D.4) implies that $y_{0}\left(\overline{\mathbf{G}}^{*}, \bar{A}_{i j}\right) \leq y_{0}\left(\underline{\mathbf{G}}^{*}, \underline{A}_{i j}\right)$.

\section{Aggregate Impact of Productivity Shocks}

Our next result is a counterpart to Theorem 9 and provides a decomposition of the aggregate impact of a productivity shock.

Theorem D.1. Consider a reduction in increase from $\underline{A}_{i j}$ to $\bar{A}_{i j}$ for a supplier-customer pair of firms $(j, i)$ and let $\overline{\mathbf{G}}^{*}$ and $\underline{\mathbf{G}}^{*}$ denote the production networks in the greatest full equilibria under $\bar{A}_{i j}$ and $\underline{A}_{i j}$, respectively. If $\underline{\mathbf{G}}^{*} \subseteq \overline{\mathbf{G}}^{*}$, then the change in aggregate output is given by

$$
\Delta \mathrm{GDP}=\left.\Delta \mathrm{GDP}\right|_{\underline{\mathbf{G}}^{*} \text { fixed }}+\left.\Delta \mathrm{GDP}\right|_{\bar{A}_{i j} \text { fixed }},
$$


where

$$
\begin{aligned}
& \Delta \mathrm{GDP}\left.\right|_{\underline{\mathbf{G}}^{*} \text { fixed }} \\
&=\operatorname{GDP}\left(\bar{A}_{i j}, \underline{\mathbf{G}}^{*}\right)-\operatorname{GDP}\left(\underline{A}_{i j}, \underline{\mathbf{G}}^{*}\right) \geq 0 \\
&\left.\Delta \mathrm{GDP}\right|_{\bar{A}_{i j} \text { fixed }}=\operatorname{GDP}\left(\bar{A}_{i j}, \overline{\mathbf{G}}^{*}\right)-\operatorname{GDP}\left(\bar{A}_{i j}, \underline{\mathbf{G}}^{*}\right) \geq 0 .
\end{aligned}
$$

Proof. The decomposition in (D.5) follows immediately from the definitions of $\Delta$ GDP $\left.\right|_{\bar{A}_{i j} \text { fixed }}$ and $\left.\Delta \mathrm{GDP}\right|_{\mathbf{G}^{*} \text { fixed }}$. It is therefore sufficient to show that both of these terms are non-negative. The fact that $\left.\Delta \mathrm{GDP}\right|_{\mathbf{G}^{*} \text { fixed }} \geq 0$ follows from Theorem 5(b): holding the set of active firms constant, an increase in productivity from $\underline{A}_{i j}$ to $\bar{A}_{i j}$ increases aggregate output. To show that $\left.\Delta \mathrm{GDP}\right|_{\bar{A}_{i j} \text { fixed }} \geq 0$, note that, by assumption, $\underline{\mathbf{G}}^{*} \subseteq \overline{\mathbf{G}}^{*}$. Furthermore, since $\overline{\mathbf{G}}^{*}$ is part of an equilibrium, all active firms $j \in \overline{\mathbf{G}}^{*}$ make non-negative net profits when the productivity is $\bar{A}_{i j}$. Therefore,

$$
\sum_{j \in \overline{\mathbf{G}}^{*} \backslash \underline{\mathbf{G}}^{*}}\left(\pi_{j}\left(\bar{A}_{i j}, \overline{\mathbf{G}}^{*}\right)-w \underline{z}_{j}\right) \geq 0 .
$$

Consequently, by Theorem $4(\mathrm{~b}), \operatorname{GDP}\left(\bar{A}_{i j}, \underline{\mathbf{G}}^{*}\right) \leq \operatorname{GDP}\left(\bar{A}_{i j}, \overline{\mathbf{G}}^{*}\right)$, thus implying that $\left.\Delta \mathrm{GDP}\right|_{\bar{A}_{i j} \text { fixed }}$ is non-negative.

In the above theorem, the assumption that $\underline{\mathbf{G}}^{*} \subseteq \overline{\mathbf{G}}^{*}$ guarantees that a positive productivity shock results in fewer failures. However, recall from Theorem 8 that, depending on the production network, positive productivity shocks may also increase failures. In such a case, the second term on the righthand side of (D.5) may no longer be unambiguously positive.

\section{Proof of Theorem 10(a)}

We first show that, given a non-negative level of aggregate demand $y_{0}$, there always exists a unique set of generic and customized quantities that clear all commodity markets.

First, we show that all customized quantities are proportional to $y_{0}$. This follows immediately from Assumption 1 (which guarantees that any feasible production network $\mathbf{G} \subseteq \mathcal{G}$ has no cycles) and the assumption that all production technologies have constant returns. Therefore, the output of any customized firm $i^{a}$ is given by $y_{i}^{a}=\alpha_{i} y_{0}$, for some constant $\alpha_{i}$ that depends on $\mathbf{G}$, the vector of prices $\left(\boldsymbol{p}^{a}, \boldsymbol{p}^{b}\right)$, and customized productivities, but is independent of all quantities.

Next, consider generic quantities. Since customized quantities are proportional to $y_{0}$, each customized producer's demand from a generic producer $j^{b}$ is also proportional to $y_{0}$. Therefore, market clearing for generic good $j^{b}$ is given by

$$
y_{j}^{b}=\beta_{j} y_{0}+\sum_{i=1}^{n} x_{i j}^{b},
$$

where $x_{i j}^{b}$ is the quantity demanded by generic producer $i^{b}$ from $j^{b}, \beta_{j} y_{0}$ is equal to the total demand for $j$ 's output from all customized firms in the economy, and $\beta_{j}$ is a constant that only depends on prices and productivities. Multiplying both sides of the above equation by $p_{j}^{b} / y_{0}$ and using the Shephard's lemma implies that

$$
\tau_{j}=\beta_{j} p_{j}^{b}+\sum_{i=1}^{n} \theta_{i j} \tau_{i}
$$


where $\tau_{j}=p_{j}^{b} y_{j}^{b} / y_{0}, \theta_{i j}=\left(p_{j}^{b} / p_{i}^{b}\right)\left(\mathrm{d} c_{i}^{b} / \mathrm{d} p_{j}^{b}\right)$, and $c_{i}^{b}$ denotes the marginal cost of generic producer $i^{b}$. It is immediate that matrix $\Theta=\left[\theta_{i j}\right]$ is element-wise non-negative. Furthermore, Euler's Theorem implies that the row sums of $\Theta$ satisfy

$$
\sum_{j=1}^{n} \theta_{i j}=\frac{1}{p_{i}^{b}} \sum_{j=1}^{n} p_{j}^{b} \frac{\mathrm{d} c_{i}^{b}}{\mathrm{~d} p_{j}^{b}} \leq \frac{c_{i}^{b}}{p_{i}^{b}}=1,
$$

where the last equality follows from marginal cost pricing of generic producers. Crucially, the assumption that labor is a direct or indirect essential input for production of all goods guarantees that, for any given $i$, either (i) the inequality above holds strictly or (ii) there is a directed path from some $j$ to $i$ such that the inequality holds strictly for $j$. This therefore guarantees that the spectral radius of $\Theta$ is strictly less than 1 , and hence $I-\Theta$ is an M-matrix. Consequently, there exists a unique non-negative vector $\tau$ that satisfies system of equations (D.6). Therefore, all generic quantities are also proportional to $y_{0}$, and in particular, $y_{j}^{b}=\tau_{j} y_{0} / p_{j}^{b}$.

So far, we showed that given aggregate demand $y_{0}$, there always exists a unique collection of quantities that satisfy market clearing for all (generic and customized) goods in the economy. The proof is therefore complete once we show that there exists a unique $y_{0}$ that satisfies labor market clearing. An argument similar to the one in the previous two paragraphs implies that the labor demand by all (generic and customized) firms in the economy is necessarily proportional to $y_{0}$. In particular, there exist constants $\hat{\alpha}_{i}$ and $\hat{\tau}_{i}$ independent of all quantities such that $l_{i}^{a}=\hat{\alpha}_{i} y_{0}$ and $l_{i}^{b}=\hat{\tau}_{i} y_{0}$. Therefore, labor market clearing implies that

$$
\sum_{i^{a} \in \mathbf{G}} \hat{\alpha}_{i} y_{0}+\sum_{i^{b}} \hat{\tau}_{i} y_{0}=L-\sum_{i^{a} \in \mathbf{G}} z_{i^{a}}
$$

Since production network $\mathbf{G}$ is feasible by assumption, there exists a unique and non-negative $y_{0}$ that satisfies labor market clearing.

\section{Proof of Theorem 10(b)-(d)}

We prove the remaining parts of Theorem 10 by showing that, in any bargaining equilibrium, the vector of equilibrium prices $\left(\boldsymbol{p}^{a}, \boldsymbol{p}^{b}\right)$ has to satisfy the system of equations described by equations (15)(17), that such a system of equations always has a solution, and that there exists a unique vector $\boldsymbol{p}^{b}$ that solves equation (15). Since the vector of generic prices $p^{b}$ is determined independently of customized prices, we first focus on characterizing these prices and establish their existence and uniqueness. We then turn to the vector of customized prices $\boldsymbol{p}^{a}$.

Generic prices: Since generic producers are competitive, the price charged by any generic producer always coincides with its marginal cost, irrespective of whether it supplies to customized, generic, or final good producers. Thus, the vector of generic prices $\boldsymbol{p}^{b}=\left(p_{1}^{b}, \ldots, p_{n}^{b}, w\right)$ is part of a bargaining equilibrium if and only if it satisfies the system of equations

$$
p_{i}^{b}=c_{i}\left(\boldsymbol{p}^{b}\right)
$$


where the function $c_{i}\left(\boldsymbol{p}^{b}\right)$ is the marginal cost of generic producer $i^{b}$ as a function of the all generic prices. ${ }^{31}$ This establishes equation (15).

We next show that this system of equations always has a strictly positive solution. Since all production functions are homogenous of degree 1, we can renormalize the vector of generic prices such that it falls within the unit simplex $\Delta^{n}$, i.e., $w+\sum_{i=1}^{n} p_{i}^{b}=1$. Let $\chi: \Delta^{n} \rightarrow \Delta^{n}$ denote the mapping

$$
\chi_{i}\left(\boldsymbol{p}^{b}\right)=\frac{c_{i}\left(\boldsymbol{p}^{b}\right)}{w+\sum_{j=1}^{n} c_{j}\left(\boldsymbol{p}^{b}\right)} .
$$

Brouwer's fixed point theorem guarantees that $\chi$ has at least one fixed point inside the unit simplex $\Delta^{n}$. Thus, there exists a vector of generic prices $p^{b}$ that solves the system of equations (15).

To show that any solution $p^{b}$ to the system of equations (D.7) is strictly positive, suppose to the contrary that there exists $\boldsymbol{p}^{b}=c\left(\boldsymbol{p}^{b}\right)$ such that $p_{i}^{b}=0$ for some generic producer $i^{b}$. This implies that (i) labor is not an essential input for the production technology of $i^{b}$ and (ii) $p_{j}^{b}=0$ for all generic producers $j^{b}$ that are essential for the production technology of $i^{b}$. This in turn implies that labor cannot be an essential input for the production technology of all producers $j^{b}$ that are essential for the production technology of $i^{b}$. Iterating this argument over the network thus implies that labor is neither directly nor indirectly an essential input for production technology of $i^{b}$, which contradicts Assumption $3^{\prime}$. Thus, any solution $p^{b}$ to the system of equations (D.7) must be element-wise strictly positive.

Finally, we establish that, in any bargaining equilibrium, the vector of generic prices is unique (up to a scaling). Suppose to the contrary that there are two distinct vectors of generic prices $\boldsymbol{p}^{b}=$ $\left(p_{1}^{b}, \ldots, p_{n}^{b}, w\right)$ and $\tilde{\boldsymbol{p}}^{b}=\left(\tilde{p}_{1}^{b}, \ldots, \tilde{p}_{n}^{b}, w\right)$ that satisfy system of equations (D.7), where note that we are normalizing the price vectors such that the wage $w$ is the same. As we already established, $p_{i}^{b}, \tilde{p}_{i}^{b}>0$ for all $i^{b}$. Let $\alpha=\min _{i} p_{i}^{b} / \tilde{p}_{i}^{b}$. We consider two separate cases depending on whether $\alpha \leq 1$ or $\alpha>1$. First, suppose $\alpha \leq 1$. By definition, it must be the case that $\alpha \tilde{p}_{j}^{b} \leq p_{j}^{b}$ for all $j^{b}$ and that that there exists $i^{b}$ such that $\alpha \tilde{p}_{i}^{b}=p_{i}^{b}$. Hence,

$$
0=c_{i}\left(p_{1}^{b}, \ldots, p_{n}^{b}, w\right)-p_{i}^{b} \geq c_{i}\left(\alpha \tilde{p}_{1}^{b}, \ldots, \alpha \tilde{p}_{n}^{b}, w\right)-p_{i}^{b}=c_{i}\left(\alpha \tilde{p}_{1}^{b}, \ldots, \alpha \tilde{p}_{n}^{b}, w\right)-\alpha \tilde{p}_{i}^{b},
$$

where the inequality is a consequence of the monotonicity of $c_{i}$ and the fact that $\alpha \tilde{p}_{j}^{b} \leq p_{j}^{b}$ for all $j^{b}$. Since $\alpha \in(0,1]$ and $c_{i}$ is concave, the above inequality implies that

$$
\begin{aligned}
0 & \geq c_{i}\left(\alpha \tilde{p}_{1}^{b}, \ldots, \alpha \tilde{p}_{n}^{b}, w\right)-\alpha \tilde{p}_{i}^{b} \geq \alpha c_{i}\left(\tilde{p}_{1}^{b}, \ldots, \tilde{p}_{n}^{b}, w\right)+(1-\alpha) c_{i}(0, \ldots, 0, w)-\alpha \tilde{p}_{i}^{b} \\
& =(1-\alpha) c_{i}(0, \ldots, 0, w) \geq 0,
\end{aligned}
$$

where the equality is the consequence of the assumption that $\tilde{p}^{b}$ satisfies (D.7). This implies that all inequalities in (D.8) and (D.9) have to hold as equalities. In particular, $c_{i}(0, \ldots, 0, w)=0$ and $c_{i}\left(p_{1}^{b}, \ldots, p_{n}^{b}, w\right)=c_{i}\left(\alpha \tilde{p}_{1}^{b}, \ldots, \alpha \tilde{p}_{n}^{b}, w\right)$. The first equality implies that labor is not an essential input for generic producer $i^{b}$, whereas the second equality implies that $\alpha \tilde{p}_{j}^{b}=p_{j}^{b}$ for all $j^{b}$ that are essential

\footnotetext{
${ }^{31}$ In particular, $c_{i}\left(\boldsymbol{p}^{b}\right)=C_{I}\left(w, p_{1}^{b} / B_{i 1}, \ldots, p_{n}^{b} / B_{i n}\right)$, where for notational simplicity, we have suppressed the productivity shocks.
} 
inputs for $i^{b} .^{32}$ But now we can iterate the above argument for all $j^{b}$ that are essential for $i^{b}$, which in turn implies that labor is not an essential input for any industry, thus contradicting Assumption $3^{\prime}$.

Next, we consider the case that $\alpha>1$, which implies that $p_{i}^{b} \geq \tilde{p}_{i}^{b}$ for all $i^{b}$. Let $\beta=\min _{i} \tilde{p}_{i}^{b} / p_{i}^{b}$. It is immediate that $\beta<1$. Now, repeating the same exact steps as above while swapping $\tilde{\boldsymbol{p}}^{b}$ and $\boldsymbol{p}^{b}$ and replacing $\alpha$ by $\beta$ results in a contradiction with Assumption $3^{\prime}$.

Taken together, the above paragraphs establish that the vector of generic prices in any bargaining equilibrium exists, is unique, and is characterized by the unique solution to the system of equations (15).

Customized prices: We now turn to customized prices. In what follows, we rely on a series of lemmas stated and proved in Online Appendix C, which characterizes the solution of pairwise bargaining between customized firms.

Let $\mathbf{G}$ denote a feasible production network of active firms. For any supplier-customer pair of active customized firms $(j, i) \in \mathbf{G}$, let $\bar{p}_{i j}=p_{j}^{b} A_{i j} / B_{i j}$ denote the price that makes the customer firm $i$ indifferent between the generic and customized variants of the good produced by industry $J$. Also, define the collection of prices $\left(\underline{p}_{i j}\right)_{(j, i) \in \mathbf{G}}$ recursively over the network $\mathbf{G}$ such that all customized firms make zero profits (gross of fixed costs of operation). ${ }^{33}$ Finally, let $P=\prod_{(j, i) \in \mathbf{G}}\left[\underline{p}_{i j}, \bar{p}_{i j}\right]$ and define the mapping $\psi: P \rightarrow P$ as

$$
\psi_{i j}\left(\boldsymbol{p}^{a}\right)= \begin{cases}c_{j} & \text { if } \pi_{i}\left(c_{j}\right)<0 \\ \hat{p}_{i j} & \text { if } \pi_{i}\left(c_{j}\right) \geq 0, f_{i j}\left(\min \left\{\bar{p}_{i j}, p_{i j}^{\dagger}\right\}\right)<0, \\ \min \left\{\bar{p}_{i j}, p_{i j}^{\dagger}\right\} & \text { if } \pi_{i}\left(c_{j}\right) \geq 0, f_{i j}\left(\min \left\{\bar{p}_{i j}, p_{i j}^{\dagger}\right\}\right) \geq 0\end{cases}
$$

where $c_{j}$ is the marginal production cost of firm $j, f_{i j}$ is the function defined in (17), $p_{i j}^{\dagger}$ is the price at which firm $i$ makes zero profits (i.e., $\pi_{i}\left(p_{i j}^{\dagger}\right)=0$ ), and $\hat{p}_{i j}$ is the solution of equation $f_{i j}\left(p_{i j}\right)=0$ in the interval $\left[c_{j}, p_{i j}^{\dagger}\right]{ }^{34}$ In Lemma C.2, we establish that there is indeed a unique $p_{i j}^{\dagger}$ such that $\pi_{i}\left(p_{i j}^{\dagger}\right)=0$ and that the interval $\left[c_{j}, p_{i j}^{\dagger}\right]$ is non-empty, whereas Lemma C.3 establishes the uniqueness of $\hat{p}_{i j} \in\left[c_{j}, p_{i j}^{\dagger}\right]$ that solves $f_{i j}\left(\hat{p}_{i j}\right)=0$.

We make two observations about the mapping defined in (D.10). First we establish that

$$
\psi_{i j}\left(\boldsymbol{p}^{a}\right) \geq c_{j}\left(\boldsymbol{p}_{-i j}^{a}\right)
$$

for any price vector $\boldsymbol{p}^{a} \in P$ and any $(j, i) \in \mathbf{G}$. To establish this claim, we consider three separate cases. First, supposes that $\pi_{i}\left(c_{j}\right)<0$, in which case (D.10) implies (D.11). As the second case, suppose that $\pi_{i}\left(c_{j}\right) \geq 0$ and $f_{i j}\left(\min \left\{\bar{p}_{i j}, p_{i j}^{\dagger}\right\}\right)<0$. Hence, $\psi_{i j}\left(\boldsymbol{p}^{a}\right)=\hat{p}_{i j}$, which, by Lemma C.2, satisfies $\hat{p}_{i j} \in\left[c_{j}, p_{i j}^{\dagger}\right]$, thus once again guaranteeing (D.11). Finally, suppose $\pi_{i}\left(c_{j}\right) \geq 0$ and $f_{i j}\left(\min \left\{\bar{p}_{i j}, p_{i j}^{\dagger}\right\}\right) \geq 0$. The monotonicity of $\pi_{i}$ (established in Lemma C.1) coupled with the fact that

\footnotetext{
${ }^{32}$ In particular, note that $\boldsymbol{p}^{b}$ and $\tilde{\boldsymbol{p}}^{b}$ are strictly positive. Therefore, if the inequality $\alpha \tilde{p}_{j}^{b} \leq p_{j}^{b}$ holds strictly for some essential input $j^{b}$, then, by definition, it must be the case that $c_{i}\left(p_{1}^{b}, \ldots, p_{n}^{b}, w\right)>c_{i}\left(\alpha \tilde{p}_{1}^{b}, \ldots, \alpha \tilde{p}_{n}^{b}, w\right)$, which contradicts the fact that (D.8) has to hold as an equality.

${ }^{33}$ In other words, vectors $\left(\bar{p}_{i j}\right)_{(j, i) \in \mathbf{G}}$ and $\left(\underline{p}_{i j}\right)_{(j, i) \in \mathbf{G}}$ are the prices that would have prevailed had the suppliers and customers had all the bargaining power in all pairwise relationships, respectively.

${ }^{34}$ Note that $p_{i j}^{\dagger}, \hat{p}_{i j}$, and $c_{j}$ all depend on the vector $\boldsymbol{p}=\left(\boldsymbol{p}^{a}, \boldsymbol{p}^{b}\right)$. We suppress this dependence for notational simplicity.
} 
$\pi_{i}\left(c_{j}\right) \geq 0=\pi_{i}\left(p_{i j}^{\dagger}\right)$ guarantees that $c_{j} \leq p_{i j}^{\dagger}$. On the other hand, note that $j$ 's marginal cost can never exceed $p_{j}^{b} \leq p_{j}^{b} A_{i j} / B_{i j}=\bar{p}_{i j}$, thus ensuring that $c_{j} \leq \bar{p}_{i j}$. Hence, $c_{j} \leq \min \left\{\bar{p}_{i j}, p_{i j}^{\dagger}\right\}=\psi_{i j}\left(\boldsymbol{p}^{a}\right)$.

As our second observation, we note that the mapping $\psi$ in (D.10) is continuous in $p^{a}$ and maps the convex and compact set $P$ to itself. Therefore, by the Brouwer's fixed point theorem, there exists a vector $\boldsymbol{p}^{a} \in P$ such that $\boldsymbol{p}^{a}=\psi\left(\boldsymbol{p}^{a}\right)$. In the remainder of the proof, we show that given any fixed point $\boldsymbol{p}^{a}$ of $\psi$, the tuple $\left(\boldsymbol{p}^{a}, \boldsymbol{p}^{b}\right)$ corresponds to a bargaining equilibrium of production network $\mathbf{G}$, where $\boldsymbol{p}^{b}$ is the unique vector of generic prices that solves the system of equations (15). In particular, we show that, under $\left(\boldsymbol{p}^{a}, \boldsymbol{p}^{b}\right)$, no customized producer has an incentive to unilaterally deviate, either by making a different offer, taking the outside option, or terminating an agreement.

Consider a fixed point $\boldsymbol{p}^{a}=\psi\left(\boldsymbol{p}^{a}\right)$. By inequality (D.11), $p_{i j}^{a}=\psi_{i j}\left(\boldsymbol{p}^{a}\right) \geq c_{j}$. Thus, under the candidate price vector $p^{a}$, all customized firms make non-negative profits. This implies that no active firm $j$ has an incentive to terminate its relationship with its downstream customer $i$. Similarly, the fact that $\boldsymbol{p}^{a}$ is a fixed point of (D.10) guarantees that no customer firm $i$ has an incentive to replace any of its customized suppliers with the corresponding generic variant. Therefore, it is sufficient to show that, given the price vector $\boldsymbol{p}^{a}$, neither the customer nor the supplier has an incentive to enter into a bargaining process. But this is an immediate consequence of Proposition C.1. In particular, recall that inequality (D.11) implies that any fixed point of $\psi$ satisfies $p_{i j}^{a}=\psi_{i j}\left(\boldsymbol{p}^{a}\right) \geq c_{j}\left(\boldsymbol{p}_{-i j}^{a}\right)$ for any pair of firms $(j, i)$, which in turn guarantees that inequality (C.1) is satisfied. This implies that the price in any fixed point of $\psi$ satisfies

$$
p_{i j}^{a}=\left\{\begin{array}{ll}
\hat{p}_{i j} & \text { if } f_{i j}\left(\min \left\{\bar{p}_{i j}, p_{i j}^{\dagger}\right\}\right)<0 \\
\min \left\{\bar{p}_{i j}, p_{i j}^{\dagger}\right\} & \text { if } f_{i j}\left(\min \left\{\bar{p}_{i j}, p_{i j}^{\dagger}\right\}\right) \geq 0
\end{array} .\right.
$$

Note that the above equation can be further simplified. In particular, the juxtaposition $f_{i j}\left(p_{i j}^{\dagger}\right)<0$ (established in the proof of Lemma C.2) with the fact that $f_{i j}$ crosses zero only once in the interval $\left[c_{j}, p_{i j}^{\dagger}\right]$ (established in Lemma C.3) guarantees that (D.12) is equivalent to $p_{i j}^{a}=\min \left\{\hat{p}_{i j}, \bar{p}_{i j}\right\}$. But this is identical to equation (C.7), which characterizes the outcome of pairwise bargaining between $i$ and $j$. Therefore, under any fixed point of $\psi$, the price $p_{i j}^{a}$ coincides with the outcome of pairwise bargaining, implying that any fixed point of $\psi$ is a vector of customized prices in a bargaining equilibrium.

\section{Proof of Theorem 11}

We first state two simple lemmas, which are invoked repeatedly throughout. We then present a sequence of results, which together establish Theorem 11. Since the statements of the results and their proofs are very similar to our main results for Leontief production technologies, we only present the counterparts to Theorems 2, 4, and 6. Throughout, we assume that Assumptions 1, 2, and $3^{\prime}$ are satisfied and that, in all pairwise interactions, the supplier has all the bargaining power, i.e., $\delta_{i j}=1$ for all supplier-customer pairs $(j, i)$.

Lemma D.5. For any feasible production network $\mathbf{G}$, there is a unique pricing equilibrium $\left(\boldsymbol{p}^{a}, \boldsymbol{p}^{b}\right)$. Furthermore, $c_{i}^{a}=p_{i}^{b}$ for all $i$ and $p_{i j}^{a}=p_{j}^{b} A_{i j} / B_{i j}$ for any supplier-customer pair $(j, i) \in \mathbf{G}$.

Lemma D.6. Let $\underline{\mathbf{G}} \subseteq \overline{\mathbf{G}}$ denote two feasible production networks and let $\eta_{i}=\pi_{i} / y_{0}$ denote the (gross) profit share of firm $i$ as a fraction of real value added. Then, 
(a) $c_{i}^{a}(\underline{\mathbf{G}})=c_{i}^{a}(\overline{\mathbf{G}})$ for all $i \in \underline{\mathbf{G}}$.

(b) $p_{i j}^{a}(\underline{\mathbf{G}})=p_{i j}^{a}(\overline{\mathbf{G}})$ for all $(j, i) \in \underline{\mathbf{G}}$;

(c) $\eta_{i}(\underline{\mathbf{G}})=\eta_{i}(\overline{\mathbf{G}})$ for all $i \in \underline{\mathbf{G}}$.

Proof. Statements (a) and (b) of the lemma are immediate consequences of Lemma D.5 and the fact that generic prices $\boldsymbol{p}^{b}$ are determined independently of the set of active customized firms. Statement (c) then follows from the previous parts and the assumption that all production technologies exhibit constant returns.

Theorem D.2 (Counterpart to Theorem 4). Let $\underline{\mathbf{G}}, \overline{\mathbf{G}} \subseteq \mathcal{G}$ denote two feasible production networks such that $\underline{\mathbf{G}} \subseteq \overline{\mathbf{G}}$. Then,

(a) $\mu_{i}(\underline{\mathbf{G}})=\mu_{i}(\overline{\mathbf{G}})$ for all $i \in \underline{\mathbf{G}}$;

(b) if condition (8) is satisfied, then $\left.y_{0}(\underline{\mathbf{G}})\right) \leq y_{0}(\overline{\mathbf{G}})$;

(c) if condition (8) is satisfied, then $\pi_{i}(\underline{\mathbf{G}}) \leq \pi_{i}(\overline{\mathbf{G}})$ for all $i \in \underline{\mathbf{G}}$.

Proof. Statement (a) is an immediate consequence of Lemma D.5. To prove statement (b), note that the representative household's budget constraint implies that

$$
\begin{aligned}
& y_{0}(\overline{\mathbf{G}})=w L+\sum_{j \in \overline{\mathbf{G}}}\left(\pi_{j}(\overline{\mathbf{G}})-w z_{j}\right) \\
& y_{0}(\underline{\mathbf{G}})=w L+\sum_{j \in \underline{\mathbf{G}}}\left(\pi_{j}(\underline{\mathbf{G}})-w z_{j}\right) .
\end{aligned}
$$

Therefore,

$$
y_{0}(\overline{\mathbf{G}})-y_{0}(\underline{\mathbf{G}})=\sum_{j \in \underline{\mathbf{G}}}\left(\pi_{j}(\overline{\mathbf{G}})-\pi_{j}(\underline{\mathbf{G}})\right)+\sum_{j \in \overline{\mathbf{G}} \backslash \underline{\mathbf{G}}}\left(\pi_{j}(\overline{\mathbf{G}})-w z_{j}\right) \geq \sum_{j \in \underline{\mathbf{G}}}\left(\eta_{j}(\overline{\mathbf{G}}) y_{0}(\overline{\mathbf{G}})-\eta_{j}(\underline{\mathbf{G}}) y_{0}(\underline{\mathbf{G}})\right),
$$

where the inequality follows from condition (8). On the other hand, Lemma D.6 guarantees that $\eta_{i}(\overline{\mathbf{G}})=\eta_{i}(\underline{\mathbf{G}})$ for all $i \in \underline{\mathbf{G}}$. As a result,

$$
\left(y_{0}(\overline{\mathbf{G}})-y_{0}(\underline{\mathbf{G}})\right)\left(1-\sum_{j \in \underline{\mathbf{G}}} \eta_{j}(\underline{\mathbf{G}})\right) \geq 0 .
$$

Since customized firms' profit shares always add up to a number strictly less than 1, the above inequality guarantees that $y_{0}(\overline{\mathbf{G}}) \geq y_{0}(\underline{\mathbf{G}})$. Finally, to establish statement (c), note that

$$
\pi_{i}(\underline{\mathbf{G}})=\eta_{i}(\underline{\mathbf{G}}) y_{0}(\underline{\mathbf{G}}) \leq \eta_{i}(\overline{\mathbf{G}}) y_{0}(\overline{\mathbf{G}})=\pi_{i}(\overline{\mathbf{G}})
$$

for all $i \in \underline{\mathbf{G}}$, where once again we are using $\eta_{i}(\underline{\mathbf{G}})=\eta_{i}(\overline{\mathbf{G}})$ as established in Lemma D.6.

Theorem D.3 (Counterpart to Theorem 2). For any network $\mathcal{G}$ of technological compatibilities,

(a) a full equilibrium exists; 
(b) the set of full equilibria has a greatest element with respect to the set inclusion order;

(c) aggregate output in the greatest full equilibrium is higher than that of all other full equilibria.

Proof. As in the proof of Theorem 2, it is sufficient to establish that mapping $\phi: 2^{\mathcal{G}} \rightarrow 2^{\mathcal{G}}$, defined as

$$
\phi(\mathbf{G})=\left\{i \in \mathcal{G}: \pi_{i}(\{i\} \cup \mathbf{G}) \geq w z_{i}\right\}
$$

satisfies Assumptions A.1 and A.2. Theorem A.1 then implies all three statements of Theorem D.3.

To establish that $\phi$ satisfies Assumption A.1, take two production networks $\mathbf{G}_{1}$ and $\mathbf{G}_{2}$ and suppose that $\mathbf{G}_{1} \subseteq \phi\left(\mathbf{G}_{2}\right)$. Hence, $\pi_{i}\left(\mathbf{G}_{2} \cup\{i\}\right) \geq w z_{i}$ for all $i \in \mathbf{G}_{1}$. Therefore,

$$
\eta_{i}\left(\mathbf{G}_{2} \cup\{i\}\right) y_{0}\left(\mathbf{G}_{2} \cup\{i\}\right) \geq w z_{i} \quad \text { for all } i \in \mathbf{G}_{1} .
$$

Furthermore, the household's budget constraint implies that

$$
y_{0}\left(\mathbf{G}_{2} \cup\{i\}\right)=w L+y_{0}\left(\mathbf{G}_{2} \cup\{i\}\right) \sum_{j \in \mathbf{G}_{2} \cup\{i\}} \eta_{j}\left(\mathbf{G}_{2} \cup\{i\}\right)-\sum_{j \in \mathbf{G}_{2} \cup\{i\}} w z_{j} .
$$

The juxtaposition of the last two equations leads to

$$
z_{i} \leq \frac{\eta_{i}\left(\mathbf{G}_{2} \cup\{i\}\right)}{1-\sum_{j \in \mathbf{G}_{2}} \eta_{j}\left(\mathbf{G}_{2} \cup\{i\}\right)}\left(L-\sum_{j \in \mathbf{G}_{2}} z_{j}\right) \quad \text { for all } i \in \mathbf{G}_{1} .
$$

On the other hand, Lemma D.6 guarantees that $\eta_{j}\left(\mathbf{G}_{2} \cup\{i\}\right)=\eta_{j}\left(\mathbf{G}_{1} \cup \mathbf{G}_{2}\right)$ for any pair $i$ and $j$ such that $j \in \mathbf{G}_{2} \cup\{i\}$ and all $i \in \mathbf{G}_{1}$. Therefore,

$$
z_{i} \leq \frac{\eta_{i}\left(\mathbf{G}_{1} \cup \mathbf{G}_{2}\right)}{1-\sum_{j \in \mathbf{G}_{2}} \eta_{j}\left(\mathbf{G}_{1} \cup \mathbf{G}_{2}\right)}\left(L-\sum_{j \in \mathbf{G}_{2}} z_{j}\right) \quad \text { for all } i \in \mathbf{G}_{1} .
$$

Next, consider the economy in which the set of active firms is instead $\mathbf{G}_{1} \cup \mathbf{G}_{2}$. In this case, firm $i$ 's gross profit is given by $\pi_{i}\left(\mathbf{G}_{1} \cup \mathbf{G}_{2}\right)=\eta_{i}\left(\mathbf{G}_{1} \cup \mathbf{G}_{2}\right) y_{0}\left(\mathbf{G}_{1} \cup \mathbf{G}_{2}\right)$. Therefore, solving for $y_{0}$ from the household's budget constraint in this economy and plugging it back into the expression for $\pi_{i}\left(\mathbf{G}_{1} \cup \mathbf{G}_{2}\right)$ leads to

$$
\pi_{i}\left(\mathbf{G}_{1} \cup \mathbf{G}_{2}\right)-w z_{i}=\frac{w \eta_{i}\left(\mathbf{G}_{1} \cup \mathbf{G}_{2}\right)}{1-\sum_{j \in \mathbf{G}_{1} \cup \mathbf{G}_{2}} \eta_{j}\left(\mathbf{G}_{1} \cup \mathbf{G}_{2}\right)}\left(L-\sum_{j \in \mathbf{G}_{1} \cup \mathbf{G}_{2}} z_{j}\right)-w z_{i}
$$

for all $i \in \mathbf{G}_{1}$. Consequently, the upper bound on $z_{i}$ in (D.14) implies that

$$
\pi_{i}\left(\mathbf{G}_{1} \cup \mathbf{G}_{2}\right)-w z_{i} \geq w \eta_{i}\left(\mathbf{G}_{1} \cup \mathbf{G}_{2}\right)\left(\frac{L-\sum_{j \in \mathbf{G}_{1} \cup \mathbf{G}_{2}} z_{j}}{1-\sum_{j \in \mathbf{G}_{1} \cup \mathbf{G}_{2}} \eta_{j}\left(\mathbf{G}_{1} \cup \mathbf{G}_{2}\right)}-\frac{L-\sum_{j \in \mathbf{G}_{2}} z_{j}}{1-\sum_{j \in \mathbf{G}_{2}} \eta_{j}\left(\mathbf{G}_{1} \cup \mathbf{G}_{2}\right)}\right) .
$$

Simplifying the right-hand side of the above inequality, we obtain,

$$
\pi_{i}\left(\mathbf{G}_{1} \cup \mathbf{G}_{2}\right)-w z_{i} \geq \frac{w \eta_{i}\left(\mathbf{G}_{1} \cup \mathbf{G}_{2}\right)}{1-\sum_{j \in \mathbf{G}_{1} \cup \mathbf{G}_{2}} \eta_{j}\left(\mathbf{G}_{1} \cup \mathbf{G}_{2}\right)}\left(\frac{\sum_{j \in \mathbf{G}_{1} \backslash \mathbf{G}_{2}} \eta_{j}\left(\mathbf{G}_{1} \cup \mathbf{G}_{2}\right)}{1-\sum_{j \in \mathbf{G}_{2}} \eta_{j}\left(\mathbf{G}_{1} \cup \mathbf{G}_{2}\right)}\left(L-\sum_{j \in \mathbf{G}_{2}} z_{j}\right)-\sum_{j \in \mathbf{G}_{1} \backslash \mathbf{G}_{2}} z_{j}\right)
$$


for all $i \in \mathbf{G}_{1}$. On the other hand, summing both sides of inequality (D.14) over all $i \in \mathbf{G}_{1} \backslash \mathbf{G}_{2}$ guarantees that the right-hand side of the above inequality is non-negative. Therefore, $\pi_{i}\left(\mathbf{G}_{1} \cup \mathbf{G}_{2}\right) \geq$ $w z_{i}$ for all $i \in \mathbf{G}_{1}$, and hence, $\mathbf{G}_{1} \subseteq \phi\left(\mathbf{G}_{1} \cup \mathbf{G}_{2}\right)$. Thus, mapping $\phi$ in (D.13) satisfies Assumption A.1.

Next, we establish that mapping $\phi$ also satisfies Assumption A.2. To this end, let $\mathbf{G}_{1}, \mathbf{G}_{2} \subseteq \mathcal{G}$ denote a pair of feasible production networks such that $\mathbf{G}_{k} \subseteq \phi\left(\mathbf{G}_{k}\right)$ for $k \in\{1,2\}$. By definition, $\mathbf{G}_{k} \subseteq \phi\left(\mathbf{G}_{k}\right)$ implies that $\pi_{i}\left(\mathbf{G}_{k}\right)=\eta_{i}\left(\mathbf{G}_{k}\right) y_{0}\left(\mathbf{G}_{k}\right) \geq w z_{i}$ for all $i \in \mathbf{G}_{k}$. Solving for $y_{0}\left(\mathbf{G}_{k}\right)$ from the household's budget constraint

$$
y_{0}\left(\mathbf{G}_{k}\right)=w L+\sum_{j \in \mathbf{G}_{k}}\left(\eta_{j}\left(\mathbf{G}_{k}\right) y_{0}\left(\mathbf{G}_{k}\right)-w z_{j}\right)
$$

therefore implies that

$$
z_{i} \leq \frac{\eta_{i}\left(\mathbf{G}_{k}\right)}{1-\sum_{j \in \mathbf{G}_{k}} \eta_{j}\left(\mathbf{G}_{k}\right)}\left(L-\sum_{j \in \mathbf{G}_{k}} z_{j}\right)
$$

for all $i \in \mathbf{G}_{k}$. Furthermore, recall from Lemma D.6 that $\eta_{i}\left(\mathbf{G}_{k}\right)=\eta_{i}\left(\mathbf{G}_{1} \cup \mathbf{G}_{2}\right)$ for all $i \in \mathbf{G}_{k}$. Hence,

$$
z_{i} \leq \frac{\eta_{i}\left(\mathbf{G}_{1} \cup \mathbf{G}_{2}\right)}{1-\sum_{j \in \mathbf{G}_{k}} \eta_{j}\left(\mathbf{G}_{1} \cup \mathbf{G}_{2}\right)}\left(L-\sum_{j \in \mathbf{G}_{k}} z_{j}\right)
$$

for all $i \in \mathbf{G}_{k}$. Setting $k=1$ and summing over all $i \in \mathbf{G}_{1} \backslash \mathbf{G}_{2}$ implies that

$$
\left(\sum_{j \in \mathbf{G}_{1} \backslash \mathbf{G}_{2}} z_{j}\right)\left(1-\sum_{j \in \mathbf{G}_{1} \cap \mathbf{G}_{2}} \eta_{j}\left(\mathbf{G}_{1} \cup \mathbf{G}_{2}\right)\right) \leq\left(L-\sum_{j \in \mathbf{G}_{1} \cap \mathbf{G}_{2}} z_{j}\right)\left(\sum_{j \in \mathbf{G}_{1} \backslash \mathbf{G}_{2}} \eta_{j}\left(\mathbf{G}_{1} \cup \mathbf{G}_{2}\right)\right) .
$$

Similarly, setting $k=2$ in (D.15) and summing over all $i \in \mathbf{G}_{2} \backslash \mathbf{G}_{1}$ implies that

$$
\left(\sum_{j \in \mathbf{G}_{2} \backslash \mathbf{G}_{1}} z_{j}\right)\left(1-\sum_{j \in \mathbf{G}_{1} \cap \mathbf{G}_{2}} \eta_{j}\left(\mathbf{G}_{1} \cup \mathbf{G}_{2}\right)\right) \leq\left(L-\sum_{j \in \mathbf{G}_{1} \cap \mathbf{G}_{2}} z_{j}\right)\left(\sum_{j \in \mathbf{G}_{2} \backslash \mathbf{G}_{1}} \eta_{j}\left(\mathbf{G}_{1} \cup \mathbf{G}_{2}\right)\right) .
$$

Next, consider the profit $\pi_{i}\left(\mathbf{G}_{1} \cup \mathbf{G}_{2}\right)$ of an arbitrary firm $i \in \mathbf{G}_{1}$ when the production network of active firms is $\mathbf{G}_{1} \cup \mathbf{G}_{2}$. Once again, solving for household demand from her budget constraint and plugging it back to firm $i$ 's profit function implies that

$$
\begin{aligned}
\pi_{i}\left(\mathbf{G}_{1} \cup \mathbf{G}_{2}\right)-w z_{i} & =\frac{w \eta_{i}\left(\mathbf{G}_{1} \cup \mathbf{G}_{2}\right)}{1-\sum_{j \in \mathbf{G}_{1} \cup \mathbf{G}_{2}} \eta_{j}\left(\mathbf{G}_{1} \cup \mathbf{G}_{2}\right)}\left(L-\sum_{j \in \mathbf{G}_{1} \cup \mathbf{G}_{2}} z_{j}\right)-w z_{i} \\
& \geq w \eta_{i}\left(\mathbf{G}_{1} \cup \mathbf{G}_{2}\right)\left(\frac{L-\sum_{j \in \mathbf{G}_{1} \cup \mathbf{G}_{2}} z_{j}}{1-\sum_{j \in \mathbf{G}_{1} \cup \mathbf{G}_{2}} \eta_{j}\left(\mathbf{G}_{1} \cup \mathbf{G}_{2}\right)}-\frac{L-\sum_{j \in \mathbf{G}_{1}} z_{j}}{1-\sum_{j \in \mathbf{G}_{1}} \eta_{j}\left(\mathbf{G}_{1} \cup \mathbf{G}_{2}\right)}\right)
\end{aligned}
$$

for all $i \in \mathbf{G}_{1}$, where the inequality is a consequence of (D.15). Simplifying the right-hand side of the above inequality therefore implies that $\pi_{i}\left(\mathbf{G}_{1} \cup \mathbf{G}_{2}\right)-w z_{i} \geq 0$ for all $i \in \mathbf{G}_{1}$ as long as $Q^{\prime}>0$, where

$$
Q^{\prime}=\left(L-\sum_{j \in \mathbf{G}_{1}} z_{j}\right)\left(\sum_{j \in \mathbf{G}_{2} \backslash \mathbf{G}_{1}} \eta_{j}\left(\mathbf{G}_{1} \cup \mathbf{G}_{2}\right)\right)-\left(1-\sum_{j \in \mathbf{G}_{1}} \eta_{j}\left(\mathbf{G}_{1} \cup \mathbf{G}_{2}\right)\right)\left(\sum_{j \in \mathbf{G}_{2} \backslash \mathbf{G}_{1}} z_{j}\right) .
$$


By inequality (D.17), we have

$$
Q^{\prime} \geq\left(\sum_{j \in \mathbf{G}_{2} \backslash \mathbf{G}_{1}} z_{j}\right)\left(\frac{L-\sum_{j \in \mathbf{G}_{1}} z_{j}}{L-\sum_{j \in \mathbf{G}_{1} \cap \mathbf{G}_{2}} z_{j}}\left(1-\sum_{j \in \mathbf{G}_{1} \cap \mathbf{G}_{2}} \eta_{j}\left(\mathbf{G}_{1} \cup \mathbf{G}_{2}\right)\right)-\left(1-\sum_{j \in \mathbf{G}_{1}} \eta_{j}\left(\mathbf{G}_{1} \cup \mathbf{G}_{2}\right)\right),\right.
$$

and as a result

$Q^{\prime} \geq\left(\frac{\sum_{j \in \mathbf{G}_{2} \backslash \mathbf{G}_{1}} z_{j}}{L-\sum_{j \in \mathbf{G}_{1} \cap \mathbf{G}_{2}} z_{j}}\right)\left[\left(L-\sum_{j \in \mathbf{G}_{1} \cap \mathbf{G}_{2}} z_{j}\right)\left(\sum_{j \in \mathbf{G}_{1} \backslash \mathbf{G}_{2}} \eta_{j}\left(\mathbf{G}_{1} \cup \mathbf{G}_{2}\right)\right)-\left(1-\sum_{j \in \mathbf{G}_{1} \cap \mathbf{G}_{2}} \eta_{j}\left(\mathbf{G}_{1} \cup \mathbf{G}_{2}\right)\right)\left(\sum_{j \in \mathbf{G}_{1} \backslash \mathbf{G}_{2}} z_{j}\right)\right]$.

Now (D.16) guarantees that the right-hand side of the above inequality is non-negative. Therefore, we just established that $\pi_{i}\left(\mathbf{G}_{1} \cup \mathbf{G}_{2}\right)-w z_{i} \geq 0$ for all $i \in \mathbf{G}_{1}$. An identical argument establishes that $\pi_{i}\left(\mathbf{G}_{1} \cup \mathbf{G}_{2}\right)-w z_{i} \geq 0$ for all $i \in \mathbf{G}_{2}$.

Consequently, we just established that $\pi_{i}\left(\mathbf{G}_{1} \cup \mathbf{G}_{2}\right)-w z_{i} \geq 0$ for all $i \in \mathbf{G}_{1} \cup \mathbf{G}_{2}$, or equivalently, $\mathbf{G}_{1} \cup \mathbf{G}_{2} \subseteq \phi\left(\mathbf{G}_{1} \cup \mathbf{G}_{2}\right)$ for any arbitrary pair of feasible production networks $\mathbf{G}_{1}$ and $\mathbf{G}_{2}$ such that $\mathbf{G}_{1} \subseteq \phi\left(\mathbf{G}_{1}\right)$ and $\mathbf{G}_{2} \subseteq \phi\left(\mathbf{G}_{2}\right)$. This implies that Assumption A.2 is satisfied.

Theorem D.4 (Counterpart to Theorem 6). In the economy's greatest full equilibrium, an increase in firm-level fixed costs

(a) shrinks the set of active firms;

(b) lowers aggregate output;

(c) reduces profits of all surviving firms.

Proof. To establish part (a), let $\bar{z}$ and $\underline{z}$ denote two vectors of fixed costs such that $\underline{z}_{i} \leq \bar{z}_{i}$ for all firms $i \in \mathcal{G}$. Define the mappings $\bar{\phi}, \underline{\phi}: 2^{\mathcal{G}} \rightarrow 2^{\mathcal{G}}$ as

$$
\begin{aligned}
& \bar{\phi}(\mathbf{G})=\left\{i \in \mathcal{G}: \pi_{i}(\{i\} \cup \mathbf{G}) \geq w \bar{z}_{i}\right\} \\
& \underline{\phi}(\mathbf{G})=\left\{i \in \mathcal{G}: \pi_{i}(\{i\} \cup \mathbf{G}) \geq w \underline{z}_{i}\right\} .
\end{aligned}
$$

It is immediate that the fixed points of $\bar{\phi}$ and $\phi$ correspond to full equilibria of the economies with fixed costs given by $\bar{z}$ and $\underline{z}$, respectively. Furthermore, recall from the proof of Theorem D.3 that $\bar{\phi}$ and $\underline{\phi}$ satisfy Assumptions A.1 and A.2. Therefore, by Theorem A.1, both mappings have a greatest fixed point, which we denote by $\overline{\mathbf{G}}^{*}$ and $\underline{\mathbf{G}}^{*}$, respectively. Thus, $\overline{\mathbf{G}}^{*}$ and $\underline{\mathbf{G}}^{*}$ are also the greatest full equilibria of the economies with fixed costs $\bar{z}$ and $\underline{z}$, respectively. On the other hand, the fact that $\underline{z}_{i} \leq \bar{z}_{i}$ for all $i \in \mathcal{G}$ implies that $\bar{\phi}(\mathbf{G}) \subseteq \underline{\phi}(\mathbf{G})$ for all $\mathbf{G} \subseteq \mathcal{G}$. Therefore, Corollary A.1 guarantees that $\overline{\mathbf{G}}^{*} \subseteq \mathbf{G}^{*}$. This establishes that increasing the vector of fixed costs shrinks the set of active firms in the economy's greatest full equilibrium.

To establish part (b), once again let $\overline{\mathbf{G}}^{*}$ and $\underline{G}^{*}$ denote the corresponding greatest full equilibria in the economies with fixed costs $\bar{z}$ and $\underline{z}$, respectively. The household's budget constraint in the two economies is given by

$$
\begin{aligned}
& y_{0}(\bar{z})=w L+\sum_{k \in \overline{\mathbf{G}}^{*}}\left(\pi_{k}\left(\overline{\mathbf{G}}^{*}\right)-w \bar{z}_{k}\right) \\
& y_{0}(\underline{z})=w L+\sum_{k \in \underline{\mathbf{G}}^{*}}\left(\pi_{k}\left(\underline{\mathbf{G}}^{*}\right)-w \underline{z}_{k}\right) .
\end{aligned}
$$


Since $\underline{G}^{*}$ is a full equilibrium, all active firms in the economy make positive net profits, i.e., $\pi_{k}\left(\underline{\mathbf{G}}^{*}\right) \geq$ $w z_{k}$ for all $k \in \underline{\mathbf{G}}^{*}$. Therefore,

$$
y_{0}(\underline{z})-y_{0}(\bar{z}) \geq \sum_{k \in \overline{\mathbf{G}}^{*}}\left(\pi_{k}\left(\underline{\mathbf{G}}^{*}\right)-w \underline{z}_{k}\right)-\sum_{k \in \overline{\mathbf{G}}^{*}}\left(\pi_{k}\left(\overline{\mathbf{G}}^{*}\right)-w \bar{z}_{k}\right) \geq \sum_{k \in \overline{\mathbf{G}}^{*}}\left(\pi_{k}\left(\underline{\mathbf{G}}^{*}\right)-\pi_{k}\left(\overline{\mathbf{G}}^{*}\right)\right),
$$

where the first inequality is a consequence of the fact that $\overline{\mathbf{G}}^{*} \subseteq \underline{\mathbf{G}}^{*}$ and the second inequality follows from the assumption that $\underline{z}_{k} \leq \bar{z}_{k}$. Denoting firm $k$ 's profit share by $\eta_{k}$, we have

$$
y_{0}(\underline{z})-y_{0}(\bar{z}) \geq \sum_{k \in \overline{\mathbf{G}}^{*}}\left(\eta_{k}\left(\underline{\mathbf{G}}^{*}\right) y_{0}(\underline{z})-\eta_{k}\left(\overline{\mathbf{G}}^{*}\right) y_{0}(\bar{z})\right) \geq\left(y_{0}(\underline{z})-y_{0}(\bar{z})\right) \sum_{k \in \overline{\mathbf{G}}^{*}} \eta_{k}\left(\underline{\mathbf{G}}^{*}\right),
$$

where the second inequality follows from Lemma D.6 and the fact that $\overline{\mathbf{G}}^{*} \subseteq \underline{\mathbf{G}}^{*}$. Since $\sum_{k \in \overline{\mathbf{G}}^{*}} \eta_{k}\left(\underline{\mathbf{G}}^{*}\right)<1$, the above inequality therefore implies that $y_{0}(\underline{z})>y_{0}(\bar{z})$.

Finally, to establish part (c) of the theorem, note that the gross profit of firm $k \in \overline{\mathbf{G}}^{*}$ under the two fixed costs is given by

$$
\begin{aligned}
& \pi_{k}(\underline{z})-w \underline{z}_{k}=\eta_{k}\left(\underline{\mathbf{G}}^{*}\right) y_{0}(\underline{z})-w \underline{z}_{k} \\
& \pi_{k}(\bar{z})-w \bar{z}_{k}=\eta_{k}\left(\overline{\mathbf{G}}^{*}\right) y_{0}(\bar{z})-w \bar{z}_{k} .
\end{aligned}
$$

Therefore,

$$
\left(\pi_{k}(\underline{z})-w \underline{z}_{k}\right)-\left(\pi_{k}(\bar{z})-w \bar{z}_{k}\right)=\eta_{k}\left(\overline{\mathbf{G}}^{*}\right)\left(y_{0}(\underline{z})-y_{0}(\bar{z})\right)+w\left(\bar{z}_{k}-\underline{z}_{k}\right),
$$

where we are once again using Lemma D.6 and the fact that $\overline{\mathbf{G}}^{*} \subseteq \underline{\mathbf{G}}^{*}$. Part (b) of the theorem guarantees that $y_{0}(\underline{z})>y_{0}(\bar{z})$ and by assumption, $\bar{z}_{k} \geq \underline{z}_{k}$. Therefore, the right-hand side of the above equation is always positive.

\section{E Online Appendix: Data Appendix}

In this appendix, we provide details on the data used in constructing Figure 1 as well as additional empirical exercises that confirm the pattern documented by that figure. Our data are drawn from the Business Dynamics Statistics (BDS) dataset from the Census Bureau (2016 release). This database is based on administrative records of U.S. firms covering the period from 1977 to 2014. BDS reports data both for firms and establishments. We use BDS reports on aggregate data by year as well as more detailed data by year, sector, and age. ${ }^{35}$

When working with aggregate data, we measure the exit rate in year $t$ as the count of firms or establishments that have ceased operations between years $t-1$ and $t$ divided by the average of the total number of firms or establishments in years $t-1$ and $t$.

When working with the detailed data, we compute firm exit rate as follows, with the establishment exit rate defined analogously. For firms aged 0-5 years, the exit rate in sector $s$ at age $a$ in year $t$ is the ratio of the number of firms that exit within sector $s$ and age group $a$ between years $t-1$ and $t$ divided

\footnotetext{
${ }^{35}$ Firms and establishments are classified into nine sectors: agricultural services, forestry, and fishing; manufacturing; construction; mining; transportation and public utility; wholesale trade; retail trade; finance, insurance, and real state; and services.
} 


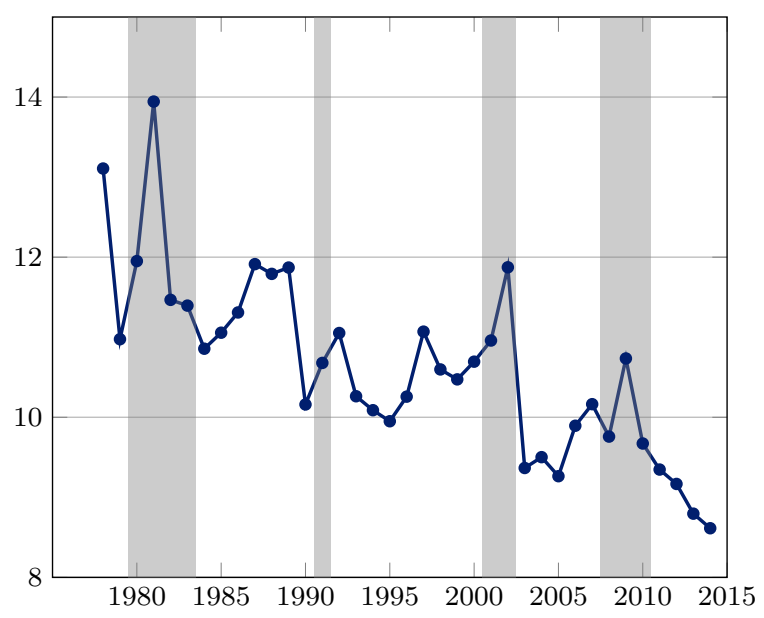

(a) raw data

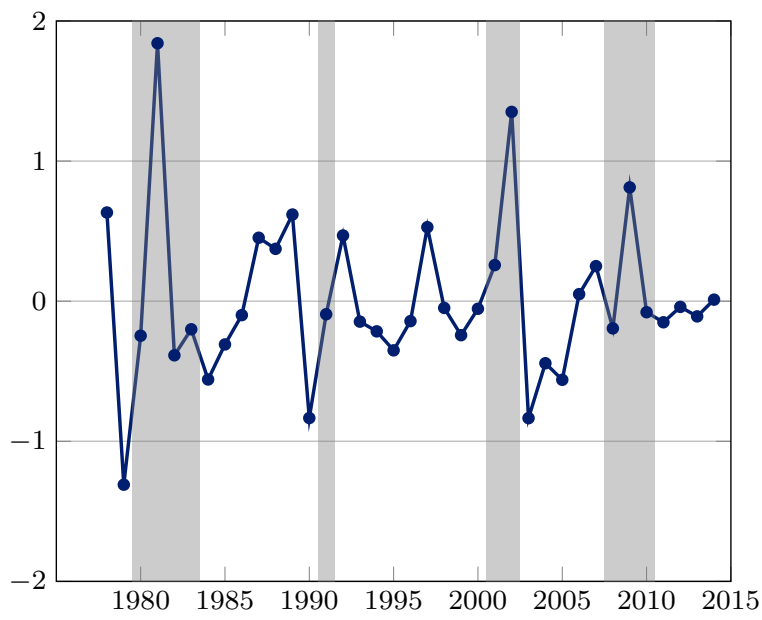

(b) HP-filtered data

Figure E.1. Annual Establishment Exit Rate in the United States over the Business Cycle

Source: Business Dynamics Statistic, United States Census Bureau.

Notes: The figure depicts the annual exit rate of establishments in the U.S. between 1978 and 2014. Exit rate in year $t$ is defined as the number of establishment failures in year $t$ divided by the average number of surviving establishments in years $t-1$ and $t$. Panel (a) plots the raw data, whereas panel (b) plots the detrended series using a Hodrick-Prescott filter with smoothing parameter 6.25. Year $t$ is shaded if there is an NBER recession at some point between the second quarter of year $t-1$ and the first quarter of year $t$.

by the average of the total number of firms within sector $s$ and age group $a$ in period $t$ and the total number of firms within sector $s$ and age group $a-1$ in period $t-1$. The BDS database groups firms aged 6 or more into five age categories: $6-10,11-15,16-20,21-25$, and 26 or more. We compute exit rates for these groups as total number of exits divided by the number of firms in this age range. ${ }^{36}$

Figure 1 plots both the raw firm exit rate and its cyclical component after removing a trend using the Hodrick-Prescott (HP) filter with a smoothing parameter of 6.25, as is standard in the literature for annual data (Ravn and Uhlig, 2002). Recession dates are from the NBER monthly business cycle indicator. ${ }^{37}$ In particular, year $t$ is coded as a recession year if any month between April of year $t-1$ and March of year $t$ has a recession according to the NBER. Figure E.1 depicts the corresponding plot for establishments.

Table E.1 confirms the patterns documented in Figures 1 and E.1 using the following simple regression on sector-age group data:

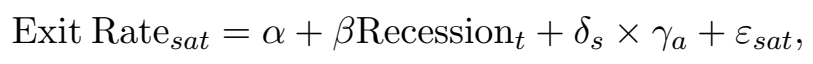

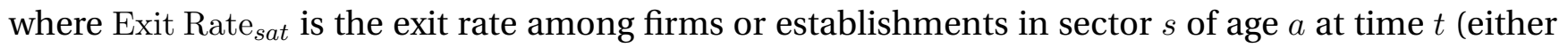
in the raw data or after removing the HP trend), $\delta_{s}$ designates a full set of sector fixed effects, and $\gamma_{a}$ stands for a full set of age fixed effects. The standard errors are clustered at the sector $\times$ age level.

\footnotetext{
${ }^{36}$ The BDS also reports an age category defined as "left censored", consisting of firms or establishments with unknown age because they were founded before 1977. We compute the exit rate for this group using an analogous strategy.

${ }^{37}$ The NBER defines a recession as a significant decline in economic activity spread across the economy, lasting more than a few months, normally visible in real GDP, real income, employment, industrial production, and wholesale-retail sales. See https://www.nber.org/cycles.html.
} 
Table E.1. Firm and Establishment Exit Rate During Recessions

\begin{tabular}{|c|c|c|c|c|}
\hline Dependent variable: & $\begin{array}{l}\text { firm exit rate } \\
\text { (1) }\end{array}$ & $\begin{array}{l}\text { firm exit rate } \\
\text { (2) }\end{array}$ & $\begin{array}{l}\text { est. exit rate } \\
\text { (3) }\end{array}$ & $\begin{array}{l}\text { est. exit rate } \\
\text { (4) }\end{array}$ \\
\hline \multicolumn{5}{|c|}{ Panel A: Raw Data } \\
\hline Recession $_{t}$ & $\begin{array}{c}0.478^{* * *} \\
(0.094)\end{array}$ & & $\begin{array}{c}1.085^{* * *} \\
(0.139)\end{array}$ & \\
\hline Recession $_{t-1}$ & & $\begin{array}{c}0.211^{* * *} \\
(0.066)\end{array}$ & & $\begin{array}{c}0.783^{* * *} \\
(0.111)\end{array}$ \\
\hline Observations & 2,619 & 2,619 & 2,619 & 2,619 \\
\hline Mean of dep. var. & 10.533 & 10.533 & 13.895 & 13.895 \\
\hline Sector $\times$ Age fixed effects & $\checkmark$ & $\checkmark$ & $\checkmark$ & $\checkmark$ \\
\hline Lin. and quad. common trends & $\checkmark$ & $\checkmark$ & $\checkmark$ & $\checkmark$ \\
\hline \multicolumn{5}{|c|}{ Panel B: HP-Filtered Data } \\
\hline Recession $_{t}$ & $\begin{array}{c}0.263^{* * *} \\
(0.048)\end{array}$ & & $\begin{array}{c}0.633^{* * *} \\
(0.065)\end{array}$ & \\
\hline $\operatorname{Recession}_{t-1}$ & & $\begin{array}{c}0.214^{* * *} \\
(0.040)\end{array}$ & & $\begin{array}{c}0.544^{* * *} \\
(0.059)\end{array}$ \\
\hline Observations & 2,619 & 2,619 & 2,619 & 2,619 \\
\hline Sector $\times$ Age fixed effects & $\checkmark$ & $\checkmark$ & $\checkmark$ & $\checkmark$ \\
\hline
\end{tabular}

Notes: Unit of analysis is sector $\times$ age $\times$ year. Exit rate in year $t$ is defined as the number of failures in year $t$ divided by the

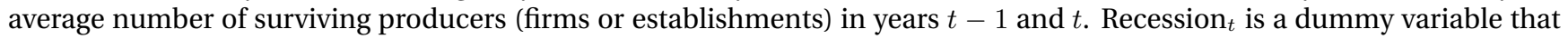
is equal to one if there is a recession in year $t$. Standard errors are clustered at the sector $\times$ age level. ${ }^{*},{ }^{* *}$, and ${ }^{* * *}$ denote significance at the $10 \%, 5 \%$, and $1 \%$ levels, respectively.

Panel A of Table E.1 reports results using the raw exit rate, while Panel B uses the HP-filtered exit rates. Regressions in Panel A additionally include quadratic time trends. The first two columns in both panels are for firms and the next two panels are for establishments, while even-numbered columns look at the lag of the recession indicator. The results confirm the patterns shown in Figures 1 and E.1: there is a strong statistical association between recessions and exits. The implied quantitative magnitudes are large as well. For example, in column 1, our estimate implies that firm exits increased by 0.48 percentage points, starting from the base of $10.53 \%$. The quantitative magnitudes are somewhat larger for establishments, corresponding to a 1.09 percentage point increase on the base of $13.90 \%$.

Table E.2 confirms these findings when observations are weighted by the average number of establishments in the relevant sector and age group. The results are very similar.

Table E. 3 reports very similar results when we use a measure of recession defined as the number of months in the recession in a given year. The coefficient estimates are not directly comparable to the previous two tables, since now we are focusing on the "intensive margin" of the recession within a given year. Nonetheless, the correlation between recession and exit rates continues to be positive and statistically significant. The implied quantitative results are also comparable: there are, on average, 6.1 
Table E.2. Firm and Establishment Exit Rate During Recessions: Weighted Regressions

\begin{tabular}{|c|c|c|c|c|}
\hline Dependent variable: & $\begin{array}{l}\text { firm exit rate } \\
\text { (1) }\end{array}$ & $\begin{array}{l}\text { firm exit rate } \\
\text { (2) }\end{array}$ & $\begin{array}{l}\text { est. exit rate } \\
\text { (3) }\end{array}$ & $\begin{array}{l}\text { est. exit rate } \\
\text { (4) }\end{array}$ \\
\hline \multicolumn{5}{|c|}{ Panel A: Raw Data } \\
\hline Recession $_{t}$ & $\begin{array}{c}0.343^{* * *} \\
(0.103)\end{array}$ & & $\begin{array}{c}0.728^{* * *} \\
(0.153)\end{array}$ & \\
\hline Recession $_{t-1}$ & & $\begin{array}{c}0.076 \\
(0.089)\end{array}$ & & $\begin{array}{c}0.394^{* * *} \\
(0.146)\end{array}$ \\
\hline Observations & 2,619 & 2,619 & 2,619 & 2,619 \\
\hline Mean of dep. var. & 10.533 & 10.533 & 13.895 & 13.895 \\
\hline Weighted mean of dep. var. & 10.112 & 10.112 & 12.934 & 12.934 \\
\hline Sector $\times$ Age fixed effects & $\checkmark$ & $\checkmark$ & $\checkmark$ & $\checkmark$ \\
\hline Lin. and quad. common trends & $\checkmark$ & $\checkmark$ & $\checkmark$ & $\checkmark$ \\
\hline \multicolumn{5}{|c|}{ Panel B: HP-Filtered Data } \\
\hline Recession $_{t}$ & $\begin{array}{c}0.164^{* * *} \\
(0.050)\end{array}$ & & $\begin{array}{c}0.430^{* * *} \\
(0.074)\end{array}$ & \\
\hline $\operatorname{Recession}_{t-1}$ & & $\begin{array}{c}0.142^{* * *} \\
(0.037)\end{array}$ & & $\begin{array}{c}0.352^{* * *} \\
(0.061)\end{array}$ \\
\hline Observations & 2,619 & 2,619 & 2,619 & 2,619 \\
\hline Sector $\times$ Age fixed effects & $\checkmark$ & $\checkmark$ & $\checkmark$ & $\checkmark$ \\
\hline
\end{tabular}

Notes: Unit of analysis is sector $\times$ age $\times$ year. Exit rate in year $t$ is defined as the number of failures in year $t$ divided by the

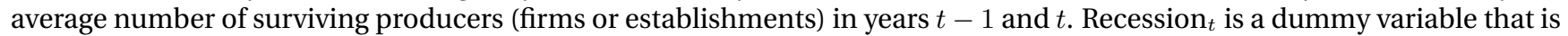
equal to one if there is a recession in year $t$. The table report results of a weighted regression, where the weight is the average number of establishments or firms by sector and age group. Standard errors are clustered at the sector $\times$ age level. *, ${ }^{* *}$, and

*** denote significance at the $10 \%, 5 \%$, and $1 \%$ levels, respectively.

recession months among years with recession periods and the coefficient estimates are about 1/6th of those in Table E.1.

We finally note that the cyclicality of exit and entry rates is also studied by Tian (2018), who concludes that "the (counter-) cyclicality in firm death is ambiguous and sensitive to the choice of cyclical indicator." This claim is based on the finding that exit rates are uncorrelated with (the cyclical component of) GDP and negatively correlated with GDP growth (see Table 1 in her paper). In the appendix (Table B-10), Tian (2018) also reports the correlation between the exit rates and several other measures to capture economic activity. Consistent with our findings, she shows that there is a positive and significant correlation between exit rates and recessions defined as the number of recession months reported by the NBER in the sample year (thus, the definition we use in Table E.3). ${ }^{38}$

\footnotetext{
${ }^{38}$ There are some minor differences between our results and Tian's (2018). First, we use a newer release of the data (version 2016) and a slightly longer period of analysis (1977 to 2014 compared to 1979 to 2013). Second, we define a recession at year $t$ based on the number of months with a recession between April of year $t-1$ and March of year $t$, whereas Tian (2018) defines a recession based on the number of months with a recession between March of year $t-1$ and February of year $t$. Third, the denominators in the definition of the exit rate in the two studies are slightly different: in our case, the denominator is the average number of firms or establishments in years $t$ and $t-1$, whereas Tian (2018) uses the number of firms or
} 
Table E.3. Firm and Establishment Exit Rate During Recessions: Intensive Margin of Recession

\begin{tabular}{|c|c|c|c|c|}
\hline Dependent variable: & $\begin{array}{l}\text { firm exit rate } \\
\text { (1) }\end{array}$ & $\begin{array}{c}\text { firm exit rate } \\
\text { (2) }\end{array}$ & $\begin{array}{l}\text { est. exit rate } \\
\text { (3) }\end{array}$ & $\begin{array}{l}\text { est. exit rate } \\
(4)\end{array}$ \\
\hline \multicolumn{5}{|c|}{ Panel A: Raw Data } \\
\hline Number of months in recession ${ }_{t}$ & $\begin{array}{c}0.054^{* * *} \\
(0.012)\end{array}$ & & $\begin{array}{c}0.134^{* * *} \\
(0.017)\end{array}$ & \\
\hline Number of months in recession ${ }_{t-1}$ & & $\begin{array}{l}0.016 \\
(0.010)\end{array}$ & & $\begin{array}{l}0.031^{* *} \\
(0.014)\end{array}$ \\
\hline Observations & 2,619 & 2,619 & 2,619 & 2,619 \\
\hline Mean of dep. var. & 10.533 & 10.533 & 13.895 & 13.895 \\
\hline Sector $\times$ Age fixed effects & $\checkmark$ & $\checkmark$ & $\checkmark$ & $\checkmark$ \\
\hline Lin. and quad. common trends & $\checkmark$ & $\checkmark$ & $\checkmark$ & $\checkmark$ \\
\hline
\end{tabular}

Panel B: HP-Filtered Data

\begin{tabular}{|c|c|c|c|c|}
\hline Number of months in recession ${ }_{t}$ & $\begin{array}{c}0.032^{* * *} \\
(0.007)\end{array}$ & & $\begin{array}{c}0.094^{* * *} \\
(0.009)\end{array}$ & \\
\hline Number of months in recession ${ }_{t-1}$ & & $\begin{array}{c}0.021^{* * *} \\
(0.008)\end{array}$ & & $\begin{array}{c}0.026^{* * *} \\
(0.009)\end{array}$ \\
\hline Observations & 2,619 & 2,619 & 2,619 & 2,619 \\
\hline Sector $\times$ Age fixed effects & $\checkmark$ & $\checkmark$ & $\checkmark$ & $\checkmark$ \\
\hline
\end{tabular}

Notes: Unit of analysis is sector $\times$ age $\times$ year. Exit rate in year $t$ is defined as the number of failures in year $t$ divided by the

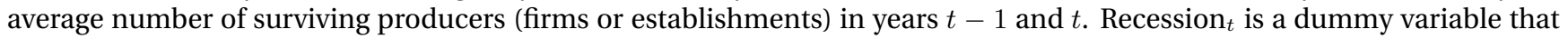
is equal to one if there is a recession in year $t$. The independent variable is the the number of months in recessions in the sample year (or its lag). Standard errors are clustered at the sector $\times$ age level. ${ }^{*},{ }^{* *}$, and ${ }^{* * *}$ denote significance at the $10 \%$, $5 \%$, and $1 \%$ levels, respectively.

In Table E.4, we show that, if instead of GDP, the focus is on significant declines in GDP, there is again a strong countercyclical relationship between GDP and exits. In Panel A, we define a significant decline in GDP as a year in which any quarter of the year experienced a more than two standard deviation decline in detrended log real GDP. Panel B provides analogous results, with the recession variable now defined on GDP growth being two standard deviations below its mean. Finally, Panel C shows a strong negative association between exit rates and GDP growth. Taken together, our findings indicate that a robustly positive relationship between recessionary periods and exit rates, i.e., exits are robustly countercyclical.

establishments in year $t-1$ as the denominator. These differences, however, have minimal effects on the results. 
Table E.4. Firm and Establishment Exit Rate During Recessions: Intensive Margin of Recession

\begin{tabular}{lcccccc}
\hline \hline \multirow{2}{*}{ Dependent variable: } & \multicolumn{2}{c}{ firm exit rate } & & \multicolumn{2}{c}{ establishment exit rate } \\
\cline { 2 - 3 } \cline { 5 - 6 } & raw data & HP-filtered & & raw data & HP-filtered \\
& $(1)$ & $(2)$ & & $(3)$ & $(4)$ \\
\hline
\end{tabular}

Panel A: Recession dummy is equal to one if GDP is below two standard deviations

\begin{tabular}{lcccc}
\hline Recession (based on GDP) & $0.531^{* * *}$ & $0.474^{* * *}$ & $0.438^{* * *}$ & $0.296^{* * *}$ \\
& $(0.114)$ & $(0.084)$ & $(0.158)$ & $(0.105)$ \\
Observations & 2,619 & 2,619 & 2,619 & 2,619 \\
Sector $\times$ Age fixed effects & $\checkmark$ & $\checkmark$ & $\checkmark$ & $\checkmark$ \\
Lin. \& quad. common trends & $\checkmark$ & & $\checkmark$ & \\
\hline
\end{tabular}

Panel B: Recession dummy is equal to one if GDP growth is below two standard deviations

\begin{tabular}{lcccc}
\hline Recession (based on GDP growth) & $1.156^{* * *}$ & $0.554^{* * *}$ & $1.270^{* * *}$ & $0.736^{* * *}$ \\
& $(0.158)$ & $(0.078)$ & $(0.213)$ & $(0.113)$ \\
Observations & 2,619 & 2,619 & 2,619 & 2,619 \\
Sector $\times$ Age fixed effects & $\checkmark$ & $\checkmark$ & $\checkmark$ & $\checkmark$ \\
Lin. \& quad. common trends & $\checkmark$ & & $\checkmark$ & \\
\hline
\end{tabular}

\begin{tabular}{lcccc}
\hline \multicolumn{5}{c}{ Panel C: GDP growth } \\
\hline GDP growth & $-14.523^{* * *}$ & $-9.217^{* * *}$ & $-22.021^{* * *}$ & $-16.481^{* * *}$ \\
Observations & $(2.704)$ & $(1.654)$ & $(3.449)$ & $(1.849)$ \\
Sector $\times$ Age FE & 2,619 & 2,619 & 2,619 & 2,619 \\
Lin. \& quad. common trends & $\checkmark$ & $\checkmark$ & $\checkmark$ & $\checkmark$ \\
\hline \hline
\end{tabular}

Notes: Unit of analysis is sector $\times$ age $\times$ year. The independent variable is defined in the header of each panel. GDP is the cyclical component of GDP. Standard errors are clustered at the sector $\times$ age level. ${ }^{*},{ }^{* *}$, and ${ }^{* * *}$ denote significance at the $10 \%, 5 \%$, and $1 \%$ levels, respectively. 